\title{
Stochastic Optimization for Emerging Wireless Networking Paradigms with Imperfect Network State Information
}

\author{
by \\ Yegui Cai \\ A thesis submitted to the \\ Faculty of Graduate Studies and Research \\ in partial fulfillment of the requirements for the degree of
}

Doctor of Philosophy in Electrical and Computer Engineering

Ottawa-Carleton Institute for Electrical and Computer Engineering (OCIECE)

Department of Systems and Computer Engineering

Carleton University

Ottawa, Ontario, Canada, K1S 5B6

July, 2014

(c) Copyright 2014, Yegui Cai 
The undersigned hereby recommends to the

Faculty of Graduate Studies and Research acceptance of the thesis

\title{
Stochastic Optimization for Emerging Wireless Networking Paradigms with Imperfect Network State Information
}

\author{
submitted by \\ Yegui Cai, B.Eng., M.Eng \\ in partial fulfillment of the requirements for the degree of \\ Doctor of Philosophy in Electrical and Computer Engineering \\ External Examiner, Professor Hai Jiang, \\ Department of Electrical and Computer Engineering, the University of Alberta
}

Thesis Supervisor, Professor F. Richard Yu

Chair, Professor Roshdy Hafez,

Department of Systems and Computer Engineering

Carleton University

July 2014 


\section{Abstract}

In wireless networks, network state information (NSI) usually consists of channel state information (CSI) and queuing state information (QSI). NSI, especially CSI, has been widely used in designs and configurations of wireless networks. However, most of the existing works assume that NSI is perfectly available at the decision making entities in the network. In general such assumption is not practical because of various limitations to acquire perfect NSI. Especially, in the context of the emerging wireless networks considered in this dissertation, it is crucial to consider imperfect NSI because it is challenging to measure and to convey perfect NSI in these systems. Due to the inaccuracy of NSI, it is challenging to make optimal decisions.

In this dissertation, we address those issues under the framework of stochastic optimizations. We first consider coordinated multi-point cellular networks with delayed CSI. The base station clustering and rate allocation problem in uplink is formulated as a networked Markov decision process, for which we derive the optimal policy with low computation cost. We further study how to provide better support for mobile cloud computing services in a cloud radio access network (C-RAN) in the second wireless networking paradigm. We formulate the problem by maximizing the system throughput while constraining the user response latency within specified values. The third wireless networking problem discussed is the resource sharing problem for software-define device-to-device communications in virtual wireless networks given 
imperfect NSI. The problem is formulated as a discrete stochastic optimization problem addressed by the proposed discrete stochastic approximation algorithms. The last type of wireless networks considered is unmanned aerial vehicle (UAV) ad hoc networks, where CSI measurements suffer from the high mobility of nodes and the challenging tactical environment. Discrete stochastic approximation based algorithms are also developed to combat the challenging operation environment of UAV ad hoc networks.

With the tools from stochastic optimizations, we can reduce the effect of imperfect NSI in those wireless networks. Extensive computer simulations are presented to show that our proposed schemes can outperform the existing schemes. 


\section{Acknowledgments}

First of all, I would like to gratefully thank my supervisor, Prof. F. Richard Yu, for his invaluable support and wonderful supervision. His technical insight and on-going encouragement have been a constant source of motivation. The innumerable discussions with him and his ideas on the research projects have been the most dispensable input for my research. His comments and suggestions on my works not only increase the quality of the research but also provide a source of thought for my career.

I would like to acknowledge Prof. Hai Jiang, Prof. Nancy Samaan, Prof. Halim Yanikomeroglu, and Prof. Minyi Huang for serving on my thesis committee.

I also would like to thank Prof. Halim Yanikomeroglu, for his valuable comments and suggestions when I was in the $5 \mathrm{G}$ research team. Besides, I want to thank Prof. Shengming Jiang, my master thesis advisor, who encouraged me to pursuit a PhD degree four years ago.

Many thanks to the fellows at Carleton University, including Mr. Ali Al-Zahrani, Mr. Zhexiong Wei, Mr. Yanwei Wang, Mr. Zhiyuan Yin, Mr. Chengchao Liang, Ms. Shengrong Bu and all other students and visitors in lab AA509. I also have the pleasure to learn a lot from talented people in the industry including Dr. Ho Ting Cheng, Dr. Taiwen Tang, Dr. Gamini Senarath and any other expert providing comments to my works. Thanks also go to friends in Ottawa, Ms. Fan Ye, Dr. Dan Chen, Mr. Steven Wang and any other individual who generously provides friendship during my years in this city. 
Moreover, I greatly appreciate my parents and my siblings for their enduring patient and understanding throughout the course of my studies. 


\section{Table of Contents}

Abstract

Acknowledgments $\quad$ v

Table of Contents vii

List of Tables xii

List of Figures xiii

List of Abbreviations $\quad$ xvi

1 Introduction 1

1.1 Motivations .......................... 1

1.2 Problem Statement . . . . . . . . . . . . . . . . . . 3

1.3 Dissertation Organization and Contributions . . . . . . . . . . . . 4

1.3.1 List of Publications . . . . . . . . . . . . . . . . . 8

2 Related Works and Prelimilaries on Stochastic Optimization 11

2.1 Coordinated Multi-Point Cellular Networks . . . . . . . . . . . . . . . 11

2.2 TCP Over Wireless Channel . . . . . . . . . . . . . . . 13

2.2.1 Splitting TCP for Mobile Cloud Computing . . . . . . . . 15

2.3 SDN and NFV in Wireless Networks . . . . . . . . . . . . 16 
2.4 Device-to-Device Communications . . . . . . . . . . . . . . . . 17

2.5 MAC for UAV ad-hoc Networks . . . . . . . . . . . . . . . . . 18

2.6 Computer Networking with Imperfect NSI . . . . . . . . . . . . . . 19

2.7 Preliminaries on Stochastic Optimizations Used . . . . . . . . . . 20

2.7.1 Networked Markov Decision Processes . . . . . . . . . . . 20

2.7.2 Discrete Stochastic Approximation . . . . . . . . . . . . 22

3 Clustering and Rate Allocation in CoMP Networks 24

3.1 Introduction . . . . . . . . . . . . . . . . . . . . 24

3.2 System Model . . . . . . . . . . . . . . . . . . 27

3.2.1 System Topology . . . . . . . . . . . . . . . . . . 27

3.2.2 Finite State Markov Chain Channel Model . . . . . . . . . . . 29

3.2 .3 Physical Layer . . . . . . . . . . . . . . . . . . . . . . . . 29

3.3 Delayed CSI Issue in Uplink CoMP . . . . . . . . . . . . . . . . 31

3.3 .1 System State Based on FSMC . . . . . . . . . . . . . . . 31

3.3 .2 Delay in CSI Sharing . . . . . . . . . . . . . . 33

3.3.3 Analysis of CoMP Based Merely on Outdated CSI . . . . . . . 35

3.4 Optimal Clustering and Rate Allocation for CoMP Systems with Delayed CSI . . . . . . . . . . . . . . . . . . . . . . . 38

3.4.1 Actions and Reward Functions . . . . . . . . . . . . 38

3.5 Belief State and Greedy Policy . . . . . . . . . . . . . . . . . . 39

3.5.1 Belief State with Known Delay Steps . . . . . . . . . . . . . . 40

3.5.2 Belief State with Unknown Delay Steps . . . . . . . . . . . . . 41

3.5.3 Greedy Policy . . . . . . . . . . . . . . . . . 42

3.6 Simulation Results and Discussions . . . . . . . . . . . . . . . 44

3.6.1 Channel Quantization ............... 46

3.6.2 Effects of Error Margin . . . . . . . . . . . . . . . . . 47 
3.6.3 Effects of Delay . . . . . . . . . . . . . . . . . 48

3.6.4 Effects of Mobility _. . . . . . . . . . . . 50

3.6.5 Discussions . . . . . . . . . . . . . . . 51

3.7 Chapter Summary . . . . . . . . . . . . . . . . . . 53

4 Dynamic Operations of C-RAN in MCC Systems 54

4.1 Introduction . . . . . . . . . . . . . . . . . . 54

4.2 System Description . . . . . . . . . . . . . . . . 58

4.2.1 Mobile Cloud Computing with Cloud Radio Access Networks . 58

4.2.2 Physical Layer and Link Layer in C-RAN . . . . . . . . . . . . 61

4.3 C-RAN with Delayed CSI . . . . . . . . . . . . . . 64

4.3.1 Finite State Markov Chain Channel Model and Delayed CSI . 64

4.3.2 Belief State with Known Delay Steps . . . . . . . . . . . . . . 65

4.3.3 Belief State with Unknown Delay Steps . . . . . . . . . . 66

4.4 TCP Throughput over C-RAN in MCC Systems . . . . . . . . . . 67

4.4.1 Round Trip Time and Split-TCP Throughput . . . . . . . . 68

4.4 .2 Per-User Response Latency _ . . . . . . . . . . . . . . . . . . 70

4.4.3 Maximizing TCP Throughput with Delayed CSI for Mobile Cloud Services . . . . . . . . . . . . . . . 71

4.4 .4 Greedy Policy . . . . . . . . . . . . . . . 72

4.5 Fairness and Efficiency Trade-off . . . . . . . . . . . . . . . 75

4.6 Simulation Results and Discussions . . . . . . . . . . . . . . 77

4.6 .1 Performance Improvement . . . . . . . . . . . . . . . . . . . 78

4.6.2 Effects of $R T T_{2}$ and $\bar{\eta}_{\text {cloud }} \ldots \ldots \ldots \ldots$. . . . . . . 82

4.6.3 Efficiency-Fairness Trade-off . . . . . . . . . . . . . 83

4.7 Chapter Summary . . . . . . . . . . . . . . . . . 84 
5 Software Defined D2D Communications

5.1 Introduction . . . . . . . . . . . . . . 87

5.2 System Description . . . . . . . . . . . . . . . . . . . . . 89

5.2.1 A Software Defined Framework Utilizing Virtual Wireless Networks ............................. 90

5.2.2 SDN Approach to Support D2D Communications . . . . . . . 91

5.2.3 Resource Sharing in a Virtual Wireless Network with D2D . . 92

5.3 Resource Sharing in Virtual Wireless Networks with Perfect Network State Information $(\mathrm{NSI}) \quad \ldots \ldots \ldots 9 . \ldots \ldots$

5.3.1 Utility Function . . . . . . . . . . . . . . . . . 96

5.4 Resource Sharing with Imperfect Network State Information (NSI) . . 97

5.4.1 Full Buffer Traffic and Imperfect CSI . . . . . . . . . . . . . . 98

5.4.2 Stochastic Traffic Arrivals and Imperfect QSI . . . . . . . 105

5.5 Simulation Results and Discussions . . . . . . . . . . . . . . 106

5.5.1 Various Traffic Arrival Intensities . . . . . . . . . . . . . 107

5.5 .2 Effect of Prices . . . . . . . . . . . . . . 108

5.5.3 Convergence Properties . . . . . . . . . . . . . . . 110

5.5 .4 Effect of NSI Noise . . . . . . . . . . . . . . . . . . 112

5.6 Chapter Summary . . . . . . . . . . . . . . . . . . . 114

6 Scheduling for UAV Ad-Hoc Networks with FDR and MPR 115

6.1 Introduction . . . . . . . . . . . . . . . . . 115

6.2 System Models . . . . . . . . . . . . . . . . . . . . . 118

6.2.1 Full-Duplex Radios (FDRs) . . . . . . . . . . . . 118

6.2.2 Multi-Packet Reception (MPR) . . . . . . . . . . . . . . 119

6.3 Transmission Optimization with Perfect Channel Knowledge . . . . . 123

6.3.1 Constraints . . . . . . . . . . . . . . . . 123 
6.3.2 Objective .......................... 125

6.3.3 Delay-Throughput Trade-off . . . . . . . . . . . . . . . . 127

6.4 Discrete Stochastic Optimization with Imperfect Channel Knowledge 128

6.4.1 Discrete Stochastic Approximation Algorithm . . . . . . . . . 128

6.4 .2 Convergence Discussion . . . . . . . . . . . . 131

6.4.3 Adaptive Step Size for Transmission Optimization in TimeVarying Channels . . . . . . . . . . . . . . . . . . 134

6.5 Simulation Results and Discussions . . . . . . . . . . . . . . 135

6.5.1 Performance Improvement . . . . . . . . . . . . . . . . . . 136

6.5.2 The Effect of Self-Interference Cancellation Factor . . . . . . . 139

6.5.3 The Effect of Channel Estimate Error . . . . . . . . . . . . . . 139

6.5.4 Convergence and Tracking the Time-Varying Channels . . . . 141

6.5.5 Chapter Summary . . . . . . . . . . . . . . 142

\section{Conclusions and Future Work 144}

7.1 Summary . . . . . . . . . . . . . . . . . . . . . . . . . 144

7.2 Future Work . . . . . . . . . . . . . . . . . . . 146

List of References 


\section{List of Tables}

3.1 Simulation Parameters for Uplink CoMP . . . . . . . . . . . . . 45

3.2 Computation Complexity Comparison . . . . . . . . . . . . 52

4.1 Simulation Parameters . . . . . . . . . . . . . . . . 78

6.1 Notation Summary in Chapter 6 . . . . . . . . . . . . . . . . . . 124

6.2 Simulation Parameters for UAV Ad-Hoc Networks . . . . . . . . . . 136 


\section{List of Figures}

1.1 The structure of this dissertation: problems and tools. . . . . . . . 5

2.1 Networked MDP with delays $[1] . \ldots \ldots$. . . . . . . . . . 21

3.1 Multi-cell multiple users scenario. . . . . . . . . . . . . . . . 28

3.2 Delay in multi-cell multi-user scenario. . . . . . . . . . . . . . . . . . 34

3.3 Raw outage probability of an uplink CoMP system consisting of three users. . . . . . . . . . . . . . . . . . . 37

$3.4 d$ steps delayed observation. . . . . . . . . . . . . . . . 40

3.5 Mean square errors versus the number of states per channel in quantization with the Lloyd-Max algorithm. . . . . . . . . . . . 46

3.6 The effects of error margins when there is no delay. . . . . . . . . . . 47

3.7 The effects of error margins when the delay is 3 steps. . . . . . . . . . 48

3.8 The effects of delay on throughput (The normalized Doppler shift is

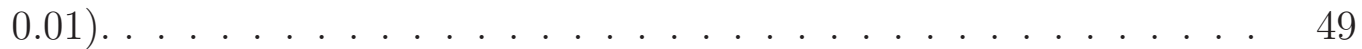

3.9 The effects of delay on throughput (The normalized Doppler shift is

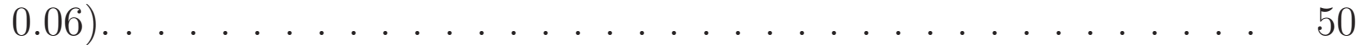

3.10 The effects of UE mobility speed on throughput when the delay is 5 steps. . . . . . . . . . . . . . . . . . . 51

3.11 The effects of UE mobility speed on throughput when the delay is 10 steps. . . . . . . . . . . . . . . . . . . . . 52

4.1 A cloud radio access network in the MCC environment. . . . . . . . . 58 
4.2 Logical protocol stacks of MCC system entities: mobile device, splitTCP proxy, and cloud backend server. . . . . . . . . . . . . 61

4.3 Round trip times and response latency using split-TCP. . . . . . . . 69

4.4 The effect of delayed CSI on the end-to-end TCP throughput in the low mobility case with normalized Doppler shift 0.01 . . . . . . . .

4.5 The effect of delayed CSI on the average response latency in the low mobility case with normalized Doppler shift 0.01. . . . . . . . .

4.6 The effect of delayed CSI on the end-to-end TCP throughput in the high mobility case with normalized Doppler shift 0.06 . . . . . . 81

4.7 The effect of delayed CSI on the average response latency in the high mobility case with normalized Doppler shift 0.06 . . . . . . . . .

4.8 The effect of response latency threshold $\alpha$ in the high mobility case with normalized Doppler shift 0.06. . . . . . . . . . . . .

4.9 The effect of $\bar{\eta}_{\text {cloud }}$ in the low mobility case with normalized Doppler shift $0.01 \ldots \ldots \ldots \ldots \ldots \ldots \ldots \ldots$

4.10 Efficiency-fairness trade-off in the low mobility case with normalized Doppler shift $0.01 . \ldots \ldots \ldots \ldots \ldots \ldots$

4.11 Efficiency-fairness trade-off in the high mobility case with normalized Doppler shift $0.06 . \ldots \ldots \ldots \ldots$. . . . . . . . . . . . 85

5.1 Virtual wireless network. . . . . . . . . . . . . . . . . . . 90

5.2 Case studies of resource sharing in virtual wireless networks. (a) The radio resource is used in $\mathrm{D} 2 \mathrm{D}$ communications for Pair $A$; (b) Pair A's uplink to InP i's network; (c) Pair A's uplink to InP j's network; (d) D2D for Pair B . . . . . . . . . . . . . . . . . . 93

5.3 Decision epochs, random traffic arrivals, and queuing dynamics in a time sequence schema. . . . . . . . . . . . . . 
5.4 Statistical distributions of the sum user utility values based on channel estimates: CSI noise variance is equal to $5 \%$ of the mean channel gains. 103

5.5 Sum user throughput performance vs. traffic arrival intensity. . . . . 108

5.6 Sum user utility performance vs. traffic arrival intensity. . . . . . . . 109

5.7 Sum user utility performance vs. the price ratio between D2D and $\operatorname{In} P_{1} .110$

5.8 Sum user utility performance vs. the price ratio between D2D and $\operatorname{In} P_{2} .110$

5.9 Sum user utility performance vs. the price ratio between $\operatorname{In} P_{1}$ and $\operatorname{In} P_{2} .111$

5.10 Sum user utility under static channels. . . . . . . . . . . . . . . . . 112

5.11 Sum user utility under time-varying channel: low CSI noise. . . . . . 112

5.12 Cumulative density function of sum user utility (full-buffer traffic and noisy CSI). . . . . . . . . . . . . . . . . . . . 113

5.13 Cumulative density function of sum user utility (Poisson arrivals, noisy CSI and QSI). . . . . . . . . . . . . . . . 114

6.1 A transmission set. . . . . . . . . . . . . . . . . . . 121

6.2 The media access control framework. . . . . . . . . . . . . . . . . 121

6.3 The structure of the token. . . . . . . . . . . . . . . . . . . 121

6.4 Statistical distributions of the objective value based on channel estimates.133

6.5 Throughput performance versus the number of UAVs. . . . . . . . . . 137

6.6 Delay performance versus the number of UAVs. . . . . . . . . . 138

6.7 The effect of self-interference cancellation factor $\psi$ on throughput. . . 140

6.8 The effect of self-interference cancellation factor $\psi$ on delay. . . . . . 140

6.9 The effect of channel estimate error rate. . . . . . . . . . . . . . . 141

6.10 Convergence of the objective values in the static channel environment. 142

6.11 Convergence of the objective values in the time-varying channel environment. . . . . . . . . . . . . . . . . . 143 


\title{
List of Abbreviations
}

\author{
ACK ACKnowledgement \\ ARQ Automatic Repeat reQuest \\ AWGN Addictive White Gaussian Noise \\ BS Base Station \\ BSC Base Station Controller \\ C-RAN Cloud-Radio Access Network \\ CDMA Code Division Multiple Access \\ CoMP Coordinated Multi-Point \\ CSI Channel State Information
}

CSMA/CA Carrier Sense Multiple Access with Collision Avoidance 
CSMA/CD Carrier Sense Multiple Access with Collision Detection

CSMA/CN Carrier Sense Multiple Access with Collision Notification

D2D Device-to-device

FDD $\quad$ Frequency Division Duplex

FDR Full-Duplex Radio

FSMC Finite State Markov Chain

HARQ Hybrid Automatic Repeat reQuest

ICI Inter-Cell Interference

LTE Long Term Evolution

LTE-A Long Term Evolution-Advanced

MAC Media Access Control

MANET Mobile Ad-Hoc NETwork

MCC $\quad$ Mobile Cloud Computing

MIMO Multiple Input Multiple Output

MMSE Minimum Mean Square Error 
MPR Multi-Packet Reception

MSE Mean Square Error

MUD Multi-User Detection

NSI Network State Information

NFV Network Function Virtualization

POMDP Partially Observable Markov Decision Process

QoS Quality of Service

RF Radio Frequency

RLC Radio Link Control

RTT Round Trip Time

SDN Software Defined Networks/Networking

SINR Signal Interference Noise Ratio

SNR Signal Noise Ratio

TCP Transmission Control Protocol

TDD Time Division Duplex

xviii 
UAV Unmanned Aerial Vehicle

UE User Equipment

UMTS Universal Mobile Telecommunication System

WTRP Wireless Token Ring Protocol 


\section{Chapter 1}

\section{Introduction}

\subsection{Motivations}

Since the commercialization of cellular communication systems, wireless communications and networks are changing our way of life. The demand for wireless technologies has moved from purely voice to extensive applications including multimedia, cloud services, internet of things, smart grid etc. A trend we can see in recent years is the convergence of traditional communication technologies (CT) and information technologies (IT) into information and communication technologies (ICT). The driving force behind this trend is mainly the fact that the earth has been evolving into a cyber-physical world. Nowadays, not only people need to communicate, machines also need to talk, and computation and communication facilities have become more and more indispensable. What we have been involving is to have such facilities as one of the signatures of the modern world a s electricity has been for ages.

From the perspective of research on wireless networks, as part of the evolving process of how technologies are changing the world, we have observed some important aspects recently. First, innovations of computation technologies are advancing the communication architecture. Take the next generation of cellular system as an 
example. Cloud computing and virtualization are expected to be an imperative elements in the design of $5 \mathrm{G}$ systems. With these two technologies, the communication infrastructures can be more energy efficient; the carriers can utilize the precious spectrum resources in a more efficient way compared with legacy systems. Second, new requirements are emerging. One of the most important questions is how to deliver the powerful cloud computing capability to the end-users. In this context, wireline networks are important elements, but wireless networks are equally important if not more. In fact, according to current statistics, there are more users accessing the Internet via smart mobile devices than from traditional personal computers. If we can say that cloud computing is the future of Internet, it might be reasonable to expect that in the future, there will be more cloud computing users accessing their services via wireless devices than via traditional computers. The third observation is on the philosophy of the networking architecture. In wireline networks, in particular, during the invention of Internet, in order to have a robust network, the control plan and the data plan are coupled. An example of the couple-ness is the Internet routing protocols, which are implemented in routers in a distributed way such that the routing decisions are made at the same entities with the forwarding actions. The trend of decoupling control plan from data plan, called software defined networking $(\mathrm{SDN})$, has been recognized both by the industry and the academia as a solution to provide flexible network designs and, in the end, to reduce the cost of innovations. Fourthly, the research advancements in wireless physical layer also introduce impacts on the design of wireless networks. Traditionally, physical layer technologies serve as the watershed between different generations of mobile networks. The new wireless physical layer technologies necessitate the introduction of resource management schemes and the new protocols because in general the legacy ones cannot fully utilize the physical layer capability. In this dissertation, we will study the emerging wireless 
networking paradigms influenced by these trends of technologies. Moreover, we also focus on a fundamental challenge in designing and configuring these systems caused by imperfect network state information.

\subsection{Problem Statement}

This dissertation focuses on the following wireless networking paradigms.

- Coordinated MultiPoint (CoMP) cellular networks: in particular, we consider the uplink joint signal processing case in CoMP. In this case, the user signals are transmitted to a central processing unit for further signal processing, in which the interference among users in multiple cells are canceled. We study the clustering and rate allocation in uplink CoMP networks.

- Cloud radio access network (C-RAN) supporting mobile cloud computing (MCC) services: C-RAN can be considered as a generalized enhancement of CoMP. This work is from another aspect on configuring C-RAN to support MCC, which is a pervasive form of mobile cloud computing integrated in the wireless environment.

- Software-defined device-to-device (D2D) communications in virtual wireless networks: SDN and wireless virtualization are two important concepts for future networking architectures. While D2D communications are envisioned to be a promising technology in next generation mobile systems, the benefits of D2D, virtualization and the application of the idea of SDN in D2D have not been fully investigated in a holistic framework.

- Unmanned aerial vehicle (UAV) ad-hoc networks with full-duplex radio (FDR) and multi-parcket reception (MPR): we investigate the transmission scheduling 
problem to exploit the FDR and MPR physical layer capabilities.

Challenges: Nonetheless, in the above wireless networking paradigms, the central research question is how to make decisions such that the capabilities of the infrastructures can be fully exploited. The information required by the decision maker (an algorithm running either in a central processing unit or in distributed nodes) is a description of the network. We call it network state information (NSI) in this dissertation. For wireless networks, the most significant element of NSI is channel state information (CSI). Since the wireless signal attenuates following a random pattern, we need to have an observation of the "actual" channel state before making any decision. The second type of NSI is the queuing state information (QSI), which is needed in decision making when the queue lengths can affect the performance evaluation. However, NSI is generally inaccurate measurement of the actual network state. In another word, due to the cost of accurate measurement, the observations are noisy or outdated version of actual states. With imperfect NSI, it is possible that the decision made is not optimal and there will be performance loss due to the sub-optimal decisions if deterministic optimization techniques are directly applied to these systems. This dissertation aims to address the wireless networking problems provided imperfect NSI in the above four wireless networking scenarios.

\subsection{Dissertation Organization and Contributions}

The structure of this dissertation is shown in Fig. 1.1. First, we devote two chapters to infrastructure-based wireless networks. In particular, we study the clustering and rate allocation schemes for CoMP cellular networks and the dynamic operations of C-RAN to support mobile cloud computing services. In both of them we address the problems related to the outdated CSI. After that, we study the intersection of the two 


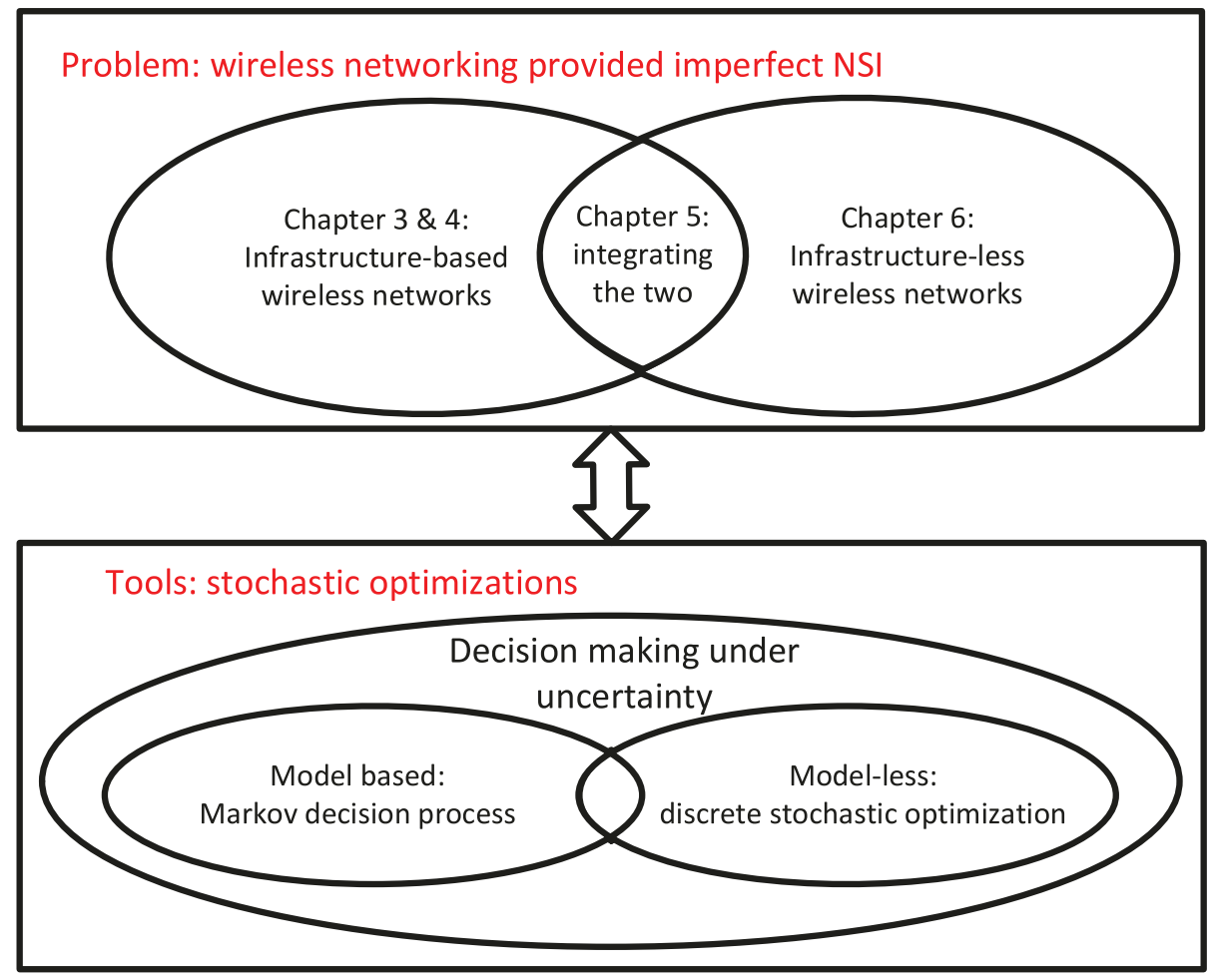

Figure 1.1: The structure of this dissertation: problems and tools.

types of networking approaches, where ad-hoc type of communications is supported in an infrastructure-based network. We introduce a new networking mechanism, software defined networking, into the system. This will make the study related to the imperfect NSI especially crucial since a global view of the whole network is required in SDN-type systems. At the end, we address the media access control (MAC) issue in an infrastructure-less wireless network where the wireless nodes are UAVs employing advanced physical layer technologies, namely, FDR and MPR capability. In the following, we detail the contributions.

In Chapter 2, we provide a brief survey of the related works in the wireless networking principles and scenarios covered in this dissertation, the related literature on networking given imperfect network state information, and a preliminary introduction to the stochastic optimization tools used in the thesis. 
In Chapter 3, we consider CoMP which is a promising technology in next generation cellular networks. Compared with traditional mobile networks, one of the important design problems in CoMP is clustering, which decides how the base stations cooperate with each other. CSI is needed in clustering decisions in CoMP. We make the following contributions:

- Most of previous works assume that perfect CSI is available. However, practical systems suffer from constraints imposed by backhaul networks, which are used for CSI exchange. In Chapter 3, we study the clustering and rate allocation problem in CoMP with delayed CSI.

- We present a decision theoretic approach to this problem. Specifically, we model such a system in the framework of networked-MDP with delays, which is equivalent to a POMDP. We derive an optimal policy for such a POMDP with low computation complexity.

- Simulation results are provided to show promising gain achieved in the proposed scheme over existing schemes especially when the delay is large and the channel coherence time is small.

In Chapter 4, we shed light on the interaction of cloud computing and wireless communications. Cloud computing will have profound impacts on wireless networks. On one hand, the integration of cloud computing into the mobile environment enables MCC systems; on the other hand, the powerful computing platforms in the cloud for radio access networks lead to the novel concept of C-RAN. In Chapter 4, we study the topology configuration and rate allocation problem in C-RAN with the objective of optimizing the end-to-end performance of MCC users in next generation wireless networks. Chapter 4 contains the following contributions: 
- An intrinsic issue related to MCC system supported by C-RAN is that only suboptimal decisions can be made due to the fact that the channel state information is outdated. We employ a decision-theoretic framework to tackle this issue, and maximize the system throughput with constraints on the response latency experienced by each MCC user.

- Using simulation results, we show that, with the emergence of MCC and CRAN technologies, the design and operation of future mobile wireless networks can be significantly affected by cloud computing, and the proposed scheme is capable of achieving substantial performance gains over existing schemes.

In chapter 5, we propose a framework for software defined D2D communications in virtual wireless networks. With software defined D2D communications, decisions such as radio resource management are made at a central controller as a piece of software. This work studies the resource sharing problem given imperfect NSI. We make the following contributions:

- Given perfect NSI, resource sharing module can decide how to utilize a pool of radio resources in different transmission modes in virtual wireless networks. However, in practical scenarios only imperfect NSI is available due to dynamic network environments and limitations in measurement.

- We formulate the resource sharing problem given imperfect NSI as a discrete stochastic optimization problem maximizing the network-wide sum utility, and develop discrete stochastic approximation (DSA) algorithms to address the stochastic optimization problem. Such algorithms can reduce the computation complexity compared with exhaustive search while achieving satisfactory performance. 
- Extensive simulations show that users' welfare can benefit from both wireless network virtualization and software defined D2D communications.

Chapter 6 addresses the challenge in the design of MAC schemes for UAV adhoc networks. The challenge is mainly due to the stringent requirements for quality of service (QoS) in complex mission environments. Most existing MAC designs for UAV ad-hoc networks are based on traditional physical layer techniques, such as halfduplex radios without multi-packet reception capability. However, recent advances in interference cancellation and signal processing techniques have enabled full-duplex radios and multi-packet reception capability, which can have significant impacts on the design of media access control schemes. In Chapter 6, we study the MAC design issues in UAV ad-hoc networks with full-duplex radios and multi-packet reception capability. To efficiently handle the highly mobile environment of a UAV ad-hoc network, a token-based technique is used for updating information in the network. Chapter 6 contains the following contributions:

- The MAC schemes in the presence of perfect and imperfect channel state information are formulated as a combinatorial optimization problem and a discrete stochastic optimization problem, respectively.

- Simulation results show that the use of full-duplex radios and multi-packet reception has significant impacts on the performance of UAV ad-hoc networks, and the proposed MAC scheme can provide substantial improvement in throughput and packet delay for UAV ad-hoc networks.

\subsubsection{List of Publications}

The following submitted or accepted papers are partially covered in this dissertation. They are in the order of chapters. 


\section{Journal and Magazine Papers}

Chapter 3: Y. Cai, F. R. Yu, and S. Bu, "A decision theoretic approach for clustering and rate allocation in coordinated multi-point (CoMP) networks with delayed channel state information," Trans. on Emerging Telecomm. Tech., DOI: 10.1002/ett.2831, April 2014.

Chapter 4: Y. Cai, F. R. Yu, and S. Bu, "When cloud computing meets wireless networks: cloud radio access networks (C-RAN) in mobile cloud computing systems," submitted to IEEE Trans. Veh. Tech., May 2014.

Chapter 4: Y. Cai, F. R. Yu, and S. Bu, "Cloud computing meets mobile wireless communications in next generation cellular networks," accepted for publication in IEEE Network Magazine, Mar. 2014.

Chapter 5: Y. Cai, F. R. Yu, and C. Liang, "Software defined D2D communications in virtual wireless networks with imperfect NSI," submitted to IEEE Trans. Veh. Tech., July 2014.

Chapter 6: Y. Cai, F. Yu, J. Li, Y. Zhou, and L. Lamont, "Medium access control for unmanned aerial vehicle (UAV) ad-hoc networks with full-duplex radios and multipacket reception capability," IEEE Trans. Veh. Tech., vol. 62, no. 1, pp. 390-394, Jan. 2013.

\section{Conference Papers}

Chapter 3: Y. Cai, F. R. Yu, and G. Senarath, "Optimal clustering and rate allocation for uplink Coordinated Multi-point (CoMP) systems with delayed channel state information (CSI)," in Proc. of IEEE ICC'13, Budapest, Hungary, June 2013.

Chapter 4: Y. Cai, F. R. Yu, and S. Bu, "When cloud computing meets wireless 
networks: cloud radio access networks (C-RAN) in mobile cloud computing systems," in Proc. of IEEE INFOCOM 2014 Workshop on Mobile Cloud Computing, Toronto, ON, Canada, Apr. 2014.

Chapter 5: Y. Cai, F. R. Yu, and C. Liang, "Resource sharing for software defined D2D Communications in virtual wireless networks with imperfect NSI," to be presented at IEEE GLOBECOM 2014, Austin, TX, Dec. 2014

Chapter 6: Y. Cai, F. R. Yu, J. Li, Y. Zhou, and L. Lamont, "Distributed scheduling for unmanned aerial vehicle networks with full-duplex radios and multi-packet reception," in Proc. of ACM DIVANet'12, Cyprus Island, Oct. 2012.

Chapter 6: Y. Cai, F. R. Yu, J. Li, Y. Zhou, and L. Lamont, "MAC performance improvement in UAV ad-hoc networks with full-duplex radios and multi-packet reception Capability," in Proc. of IEEE ICC'12, Ottawa, ON, Canada, June 2012.

\section{Patent Application}

Chapter 3: Y. Cai, F. R. Yu, G. Senerath, and H. Yanikomeroglu, "System and method for controlling multiple wireless access nodes," Filed by Huawei, Canada, US patent application no: 14/292,483, application date: 31 May 2014. 


\section{Chapter 2}

\section{Related Works and Prelimilaries on}

\section{Stochastic Optimization}

This chapter provides a review of the literature related to this dissertation.

\subsection{Coordinated Multi-Point Cellular Networks}

Interference management has been undergoing a shift from traditional static resource partitioning to dynamic coordination and cooperation schemes [2]. Among those interference management techniques, one of the most promising ones is coordinated multi-point (CoMP) [3,4] proposed in the third Generation Partnership Project (3GPP) Long-Term Evolution-Advanced (LTE-Advanced). CoMP is also known as network multiple input multiple output (MIMO) or base station (BS) cooperation, and similar concepts can be traced back to distributed antenna systems [5] or macrodiversity in CDMA age [6]. CoMP exploits ICI by allowing channel state information (CSI) and/or user data to be shared and jointly processed by multiple base stations $[3,7]$. Significant capacity gain is predicted if perfect CSI of the desired and interfering signals are available at the cooperating BSs or at a central controller.

In CoMP, one of the important system design problems is clustering, which decides 
the pattern of cooperating base stations. There are two types of clustering approaches, namely, static clustering and dynamic clustering, in the current literature. Both types of clustering approaches require CSI to be either shared among network entities or to be collected at a central controller. In the past few years, several algorithms for static or dynamic clustering in CoMP have been proposed. Papadogiannis et al. show that dynamic clustering with a cluster consisting of 2 cells outperforms static clustering with much lager cluster size [8]. A decentralized framework for downlink CoMP is proposed in [9]. Both dynamic clustering and power allocation are considered in their work. The authors of [10] present a dynamic clustering algorithm by maximizing the total system capacity for uplink transmission of multi-user distributed antenna systems. With the choice of different CSI representations, their proposed algorithm achieves a tradeoff between the performance gain and joint processing complexity. Moon and Cho propose a heuristic algorithm to solve the combinatorial cell-clustering problem to increase the sum-rate of all users [11]. Recent works on dynamic clustering in CoMP also include [12], where a systematic framework is proposed to address both channel randomness and queueing dynamics. General studies on signal processing techniques and scheduling in CoMP are also abundant in the literature (e.g., [13] and [14]).

In the context of adaptive signal processing, the effect of imperfect CSI has been studied extensively. An overview of the techniques for communication systems with limited feedback can be found in [15] and the references therein. The focus of the research there is to quantize and to compress CSI in order to avoid sacrificing performance. More recently, Bhagavatula and Heath focus on the resource allocation for CSI feedback in multi-cell systems [16]. Their closed-form bit partitioning scheme suggests allocating more bits to quantize the stronger channels with smaller delay and fewer bits to weaker channels with larger delay. In the context of CoMP, Marsch 
and Fettweis study the decoding strategies for uplink CoMP under a constrained backhaul and imperfect CSI [17]. The effect of signalling delay on the performance of centralized scheduling is investigated in [18] and [19]. The system considered is a joint detection cooperating system. Their results show that with the increase of user mobility, the system performance degrades due to the inaccuracy of outdated CSI. To address this issue, statistical CSI is used for rate allocation. Huang et al study the effect of feedback delay on a transmit beamforming system in [20] and [21]. They show that the ergodic capacity with respect to the case of no feedback delay deceases exponentially with feedback delay. Notably, they model the channel as a finite state Markov chain, which leads to elegant results about the relationship between channel capacity and delay in CSI.

Another stream of related research is on predicting fading channels. The major objective there is to find the most likely channel states based on outdated estimates. The likelihood is defined on some criteria, e.g., minimum mean square error. A survey of the state-of-the-art can be found in [22] and references therein. Similar to the literature on the limited feedback, the majority of that line of research focuses on the signal processing side.

\subsection{TCP Over Wireless Channel}

There are two lines of research in the past on improving the end-to-end performance of TCP. In the first one, many researchers propose to change the behavior of TCP to overcome its limitations over wireless channel. A recent survey can be found in [23]. In the second one, researchers focus on dynamically configure wireless networks such that TCP's performance can be optimized. Since the work in Chapter 4 lies in the second category. We will briefly introduce this line of research.

Alcaraz et al study the Reliable Link Layer (RLC) protocol in UMTS which 
adopts ARQ to support reliable data transmission in [24]. They discuss the interaction between TCP and RLC in the third generation wireless networks, and suggest optimum parameter configurations at both layers. The authors of [25] discuss the necessity of link layer retransmission mechanisms to improve the TCP performance. They find out that it is not easy to analyze TCP using the queueing theory because of TCP's feedback mechanism. In [26] Toledo et al analyze TCP's performance over MIMO channel. They propose an iterative procedure to analyze TCP throughput with queueing delay. Specifically, they first compute the TCP throughput without delay which serves as an upper bound. Then they calculate the delay and packet loss probability due to limited queue length. The cycle repeats itself until the throughput converges to a fixed value. Different to [26], the authors of [27] consider TCP dynamics instead of the ergodic behavior of TCP. A dynamic programming based approach is proposed to maximize TCP throughput. Note that the dynamic behavior of TCP is quite involved to be captured by a mathematical model. Assaad et al analyze the effect of TCP on UMTS-HSDPA system, in particular, scheduling in [28]. They proposed a scheme to guarantee TCP flow rates without losing cell capacity.

More recently, [29] and [30] address how to select the optimal rate out of two channel rates to maximize the throughput in CDMA networks. Their motivation is that the original trade-off between data rates and frame error rates designed for inelastic traffic such as voice will not be the best for TCP. For different stage of a TCP flow, a particular channel rates would be best. For instance, in slow state, a higher data rate may be desirable since packet error in this stage is not the biggest concern. The cross-layer design in a cognitive ratio network can also find in [31] where various link layer parameters are considered. In [32] the congestion level is abstracted as congestion factor, which is unknown. But an optimal policy can be learned in the POMDP formulation. We can consider this work as an enhancement to the original 
TCP for video traffic since the policy derived is a sequence of congestion window update actions.

Among these papers, an important issue is how to model wireless channel. In [25], [33], and [26] a two-state Markov chain model is used. In such model, the bad state corresponds to a link failure, namely, frame error, or, outage; otherwise it is the good state. These papers either model the channel itself as a Markov chain, for instance, [31] or model the wireless link taking the implementation of the physical layer into consideration as a Markov chain.

\subsubsection{Splitting TCP for Mobile Cloud Computing}

The benefits of splitting a TCP flow into two connections have been well recognized [34] [35] in literature of both wireless networks and cloud computing, separately. Indeed among the enhancements of TCP for wireless networks, split-TCP is the mostly widely used one [34]. Split-TCP hides the wireless related issue from the wireline host via inserting a split point between wireless and wired hosts. The split point, called proxy, locally acknowledges each segment and then stored and forwarded the segment on the second TCP connection. So the single end-to-end TCP connection becomes two connections, one between the wired host and the split point and the other one between the split point and the wireless host. Such approach has been shown to be deployed in commercial cellular networks [34]. Baccelli et al study

the dynamics of split-TCP in [36]. They establish the explicit relationship between throughput dynamics and the buffer occupancy in the proxy.

In the context of cloud computing, split-TCP is also a popular scheme to provide reliable data transfer for cloud services. The client sets up a TCP connection to the nearby split-TCP proxy, then the split-TCP proxy sustains a persistent TCP connection to the data center with a very large TCP connection window. The authors 
of [37] use machine learning and statistical inference to address the "what-if" deployment and configuration problem in contend distribution networks (CDNs), with the objective to minimize the response time of web applications such as search engine. In [35] Pathak et al discuss the benefits of split-TCP in the world-wide network consisting of satellite datacenters and mega datacenters. Chen et al develop an inference framework to measure and quantify the factors which cannot be explicitly observed and measured at the end hosts in [38]. Either split-point, proxy, or front-end, refers to the same network element.

\subsection{SDN and NFV in Wireless Networks}

Recently, software defined networking (SDN) has been widely considered as a novel approach to promote innovations in communication networks $[39,40]$. In wireless networks, by identifying and abstracting the wireless primitives and functions, wireless SDN can provide mechanisms for developing innovative wireless networking methodologies [41]. There have been some works on applying the SDN concepts in wireless networks ranging from radio access networks to core mobile networks, and from control plane to data plane. SoftRAN [42] and CellSDN [43] are software defined control planes for radio access networks by abstracting all the access points in a local geographical region as a virtual big-access point. OpenRadio [44] provides a solution for programmable wireless data plane. For core networks, SoftCell [45] directs traffic to traverse a sequence of middle-boxes while optimizing the performance according to network conditions. MobileFlow [46] is a concrete architecture for SDN in mobile networks, in which a whole mobile network is re-factored into a virtual network management framework.

Another promising technology is network function virtualization (NFV) [39, 40, 
47]. In wireless networks with virtualization, network infrastructure can be decoupled from the services that it provides [48]. Consequently, multiple wireless virtual networks operated by different service providers (SPs) can dynamically share the physical substrate wireless networks operated by mobile network operators (MNOs). Since wireless network virtualization enables the sharing of infrastructure and radio spectrum resources, the capital expenses (CapEx) and operation expenses (OpEx) of wireless (radio) access networks (RANs), as well as core networks (CNs), can be reduced significantly. Moreover, mobile virtual network operators (MVNOs) who may provide some specific telecom services can help MNOs attract more users, while MNOs can produce more revenue by leasing the isolated virtualized networks to them [49]. A recent survey about wireless network virtualization can be found in [50].

\subsection{Device-to-Device Communications}

D2D communications can be used to satisfy the demand for local traffic such as peer-to-peer file sharing and machine-type communications [51,52]. Meanwhile, D2D may result in new types of wireless peer-to-peer services [53] and it can serve for the purpose of offloading the traffic from radio access networks [54]. In terms of improving the systematic performance of mobile networks, single hop D2D communications have the following benefits $[55,56]$. First, reuse gain allows radio resources to be simultaneously used by both cellular and D2D links. Second, proximity gain allows high bit rates, low latency and low power consumption thanks to the locations of the two nodes in the D2D link. Third, hop gain enables using a single link rather than using uplink and downlink, which results in two hops communications. Even multi-hop D2D communications [57] is still of interest, we only consider single-hop D2D communications in this work since its benefits have been widely recognized.

Resource sharing is among the challenges to introduce D2D communications into 
cellular networks [55]. In addition to traditional radio resource management techniques such as power control and resource allocation, we need to consider mode $s$ election in such wireless networks. Mode selection schemes aim to select the best transmission mode to transfer data in a potential D2D pair. Traditionally, the mode selection problem is addressed by deciding a threshold to switch between relying the data flow via access points or transmitting the data directly between mobile users. And the metrics for the threshold usually include the distance between the transmitter and the receiver of the potential D2D pair [58], the expected received SINR [59], or the achievable data rates [60]. A comprehensive survey of the radio resource management techniques of D2D communications can be found in [61].

\subsection{MAC for UAV ad-hoc Networks}

A major challenge in UAV ad-hoc networks lies on the medium access control (MAC) design, which is critical in guaranteeing low communication latency, low packet error rate, and high throughput. Since existing MAC protocols are designed for generic MANETs and sensor networks, they do not account for the characteristics of UAV adhoc networks, and thus may not be appropriate for such networks. For example, the contention-based random access MAC protocols widely used in MANETs [62], such as those based on carrier sense multiple access with collision avoidance (CSMA/$\mathrm{CA}$ ), can cause long and variable latency due to the hidden terminal and exposed terminal problems in UAV networks [63]. It has been shown that token-based MAC schemes outperform contention-based MAC schemes in some aspects, and has many advantages for wireless ad-hoc networks [64-66]. Some notable advantages include robustness against single node failure and support for flexible topologies, in which nodes can be partially connected without being connected to the access point. Because of its advantages, token-based MAC protocols for ad-hoc networks have been 
gaining popularity in recent years [65].

Recently, some works have been done for MAC protocols in UAV ad-hoc networks. In [63], the authors proposed a MAC protocol for UAV-to-ground communications using CDMA for data transmission. A MAC protocol, referred to as the Centralized Intelligent Channel Assigned Multiple Access (C-ICAMA) protocol, is proposed in [67] for ground mobile backbone nodes to access a UAV based on the asymmetric traffic patterns in tactical environments. Lin et al proposed a link layer scheme based on the use of multiple transmitters and receivers to achieve higher TCP goodput [68].

\subsection{Computer Networking with Imperfect NSI}

As mentioned before, NSI for wireless networks consists of CSI and QSI. There have been a considerable amount of works on addressing the imperfect CSI at physical layer. An overview of the techniques for wireless communication systems with limited feedback can be found in [15] and the references therein. The focus of the research there is to quantize and to compress CSI in order to avoid sacrificing performance. The related works on the impact of imperfect CSI on CoMP systems can be found in Section 2.1.

As for the literature related to imperfect NSI, the number of papers is comparably small. The authors of [69] study the imprecise topology state information in optical networks, where the maintenance of global topology information is impossible. A QoS routing algorithm with imperfect NSI is proposed in [70]. The main inaccuracy in NSI therein is in the computation of QoS metrics. Their main focus is also in the topology database used for path selection. The inaccuracy of the topology database is described through a probabilistic model. Another relevant work is [71], which addresses the theoretical aspect of delayed NSI in wireless networks. However, the authors only consider the network capacity of very simple network topologies given 
imperfect NSI.

\subsection{Preliminaries on Stochastic Optimizations Used}

We use stochastic optimization techniques in addressing the issues related to imperfect NSI. Mathematically, uncertainty is characterized by probability, while the problems related to decision making under uncertainty can be formulated as stochastic optimizations. Therefore, mathematical formulations modeling uncertainty are useful for addressing the issues caused by imperfect NSI. The two models we borrow from the stochastic optimization literature are networked-Markov decision processes and discrete stochastic approximation, which belong to model-based and model-less formulations, respectively. In model-based formulations, a particular stochastic feature about the system states, such as Markovian property, is assumed. While in model-less formulations, there are no assumptions on the randomness of the system states.

\subsubsection{Networked Markov Decision Processes}

Markov decision processes (MDPs) describe decision making under stochastic systems which can be partly controlled by the decision maker [72]. The stochastic systems are represented as Markov chains, in which the future state of the system is independent of the past one if the present of the system state is given. Mathematically, MDPs are discrete time stochastic control processes with state space $\mathcal{S}$, action space $\mathcal{A}$ and reward/cost function $R(\cdot)$. Suppose at the current slot the system state is $s \in \mathcal{S}$ and the action taken at the beginning of next slot is $a \in \mathcal{A}$, the system state will jump to the next state value $s^{\prime} \in \mathcal{S}$ with probability $\operatorname{Pr}\left(s^{\prime} \mid s, a\right)$ and the reward or cost received 


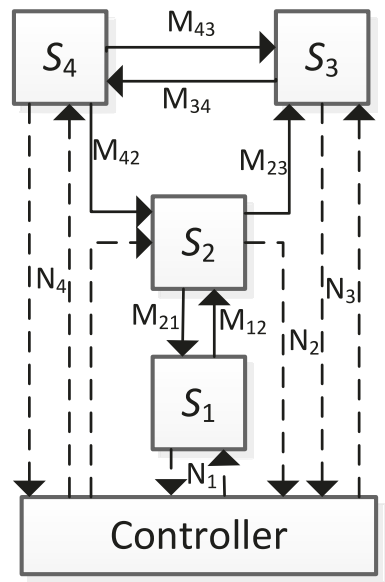

Figure 2.1: Networked MDP with delays [1].

will be $R\left(a, s, s^{\prime}\right)$. The ultimate goal of MDPs is either to minimize the cost or to maximize the reward over a period of time. In MDP literature, the time is in unit of horizon. If we consider infinite time, the corresponding MDPs are called infinite horizon MDPs, otherwise finite horizon MDPs.

If we want to model a system consisting multiple MDPs, and between the subsystems, the communication is not perfect, we can use networked-MDPs $[1,73]$. Fig. 2.1 illustrates a networked-MDP system with 4 subsystems, $S_{1}, S_{2}, S_{3}, S_{4}$ and a central controller. The delays between the subsystems and the controller are denoted as $N_{i}, i \in\{1,2,3,4\}$, and the delays between two subsystems $i$ and $j$ are denoted as $M_{i, j}$. If we assume there is a central controller, Adlakha et al have proven that the networked-MDP is equivalent to a single big MDP with partially observations which is coined Partially Observable Markov Decision Process (POMDP) [1,73].

In POMDP, the central controller cannot see the actual system state, instead only noisy or delayed observations are available. The set of all the possible observation values are usually denoted as an observation space $\mathcal{O}$. In a well-defined POMDP, the probabilistic relation between actual system state and an observation is defined as observation function [74]. 


\subsubsection{Discrete Stochastic Approximation}

Suppose we need to solve the following discrete stochastic optimization problem [75]

$$
\max _{n \in \mathcal{N}} f(n)=\mathbb{E}\left[X_{n}\right]
$$

in which $\left\{X_{n}\right\}$ is a sequence of random variables, $n \in \mathcal{N}$ is an action from a finite number of different alternatives. When the expected values $\mathbb{E}\left[X_{n}\right], n=1, \ldots, \ldots N$, have analytical forms, problem (2.1) can be solved via standard integer programming solvers. However, if the objective function cannot be evaluated explicitly, and only noisy observations are available, those solvers cannot be applied. Such optimization problems are also called simulation optimization [76], in which the main focus is to decide the optimal settings for a simulation model. Nonetheless, as we will see in the rest of the dissertation, the tools from the simulation optimization domain provide novel approaches to address the issues in wireless networks with imperfect NSI.

A straightforward method to solve discrete stochastic optimization (2.1) is the socalled exhaustive search procedure. To do so, we first need to compute the empirical average of the objective function assuming a particular action $n$ is taken. Over a very large period of time $T$, according to strong law of large numbers, we have $\hat{f}(n) \triangleq$ $\frac{1}{T} \sum_{t=1}^{T} f(n) \rightarrow \mathbb{E}[f(n)]$. Then the optimal action is

$$
x^{*}=\underset{n \in \mathcal{N}}{\operatorname{argmax}} \hat{f}(n) .
$$

The exhaustive search approach has two disadvantages. First, it requires an extremely large amount of computation. To be exact, the number of times computing the objective function value is $T \cdot|\mathcal{N}|$. Even if it is not possible to do so, the efficiency of the computation is very low because ideally only the empirical average of the optimal objective function is needed. Second, the actual wireless network is dynamic 
system and the channel states are time varying. But to have statistically meaningful empirical average, we need to have a sufficiently large $T$, which is not achievable if the channels are changing in a time period less than $T$.

Several algorithms have been proposed to solve (2.1). In general, when the feasible set $\mathcal{N}$ is small (usually less than 30), ranking and selection [77] and multiple comparison procedures [78] are practical; If the feasible set is large we have to turn to random search, e.g., [75]. The discrete stochastic approximation (DSA) algorithms used in this dissertation are in the category of random search and are based on the works in $[75,79]$. These procedures generate a homogeneous Markov chain taking values from $\mathcal{N}$ which spends more time at the global optimal than at any other member in $\mathcal{N}$. A survey of those techniques and procedures can be found in [76]. 


\section{Chapter 3}

\section{Clustering and Rate Allocation in CoMP Networks}

\subsection{Introduction}

The next generation wireless networks are envisioned to meet higher and higher demand for data rates to support sophisticated service such as cloud computing. To this end, the current systems are adopting more and more aggressive resource reuse, which inevitably results in severe co-channel interference $[2,80]$. Besides, with the advent of the heterogeneous networks (HetNet) concept [80,81], the co-channel interference can be even worse since the network deployment may simply follow an ad hoc style [82]. In a nutshell, although both of these two trends can increase the network capacity, they raise big challenges in interference management. One particular issue to be addressed is the severe inter-cell interference (ICI) for cell-edge users.

Although some excellent works have been done on CoMP, most of them assume that perfect CSI is available. However, in practical systems, CSI is imperfect due to the imperfectness in channel estimation and the delay in transmitting CSI. In CoMP, such issue is getting more serious, because CSI is propagated over backhaul networks, which are constrained in capacity, and introduce lost and delayed CSI. For 
example, in LTE-Advanced, the standard interface for inter-BS communications, $X 2$, is designed to allow a latency of $20 \mathrm{~ms}$ for control plan messages, and the typical value for the latency is expected to be $10 \mathrm{~ms}$ on average [83]. The CoMP performance can be degraded significantly due to the CSI packet delay and losses over the backhaul networks $[18,84]$.

On one hand, imperfect CSI has significant impacts on the performance of not only CoMP cellular networks, but also wireless networks in general. Indeed, the capacity of channels with imperfect CSI is largely unknown in wireless networks. It is difficult, if not impossible, to solve this problem using traditional information theoretical approach due to the limitations of Shannon Theory [85]. On the other hand, stochastic control/decision theories have well-developed mechanisms to address the impacts of noise and delay in the channels, which will give insights into the problems related to imperfect CSI from a new perspective $[85,86]$.

In this chapter, we take a decision theoretic approach for cooperative wireless cellular networks with CoMP communications. Some distinctive features of the proposed scheme are as follows.

- In contrast to previous works that assume perfect CSI, we study the optimal clustering and rate allocation problem for CoMP systems with delayed CSI. To make clustering and rate allocation decisions, each BS needs to send the local CSI to a central controller. Due to the delay in channel estimation and transmission over backhaul networks, there is a gap between the exact channel state and the observed CSI, based on which the central controller clusters the BSs and allocates user equipment (UE) transmission rates.

- We model the wireless channel as a Markov chain. The effect of delay is investigated in this model. Although Markov channel models have been widely 
accepted in the literature as an effective approach to characterize wireless channels [87-92], few works have used such models in addressing the issues caused by system delay, especially delay in backhaul networks.

- We formulate the clustering and rate allocation problem as a networked Markov decision process (networked-MDP) with delays $[1,73]$. In networked-MDP, the whole system consists of multiple subsystems. The states of each subsystem are characterized by a Markov chain. Actions in subsystems can partially control the evolution of each subsystem. Here the subsystem is a base station in CoMP cellular networks.

- We show that the networked-MDP model with delay is equivalent to a partial observable Markov decision process (POMDP) [74]. In POMDP, the whole system is characterized by a Markov chain but only noisy observations of the actual system states are available. For the CoMP system in this work, we mainly work on the inaccuracy of observations caused by delayed CSI. Furthermore, by exploiting the structure of our formulation, we design a low complexity optimal policy for clustering and rate allocation.

- Extensive simulation results show that our proposed scheme outperforms existing schemes under various settings, including different delay and UE mobility settings.

The rest of the chapter is structured as follows. Section 3.2 describes the system model. Section 3.3 discusses how the delay comes and the effects of delayed CSI on clustering and rate allocation. We formulate the problem and propose a solution in Section 3.4 and Section 3.5, respectively. Simulation results are discussed in Section 3.6. Finally we conclude this work in Section 3.7.

Notation: In this chapter $\mathbf{X}$ refers to a matrix and $\mathbf{x}$ refers to a vector. The 
cardinality of a set $\mathcal{S}$ is represented as $|\mathcal{S}|$. The transpose and Hermitian of $\mathbf{X}$ are given by $\mathbf{X}^{T}$ and $\mathbf{X}^{*}$, respectively. $\mathbf{I}_{K}$ is a $K$-by- $K$ identity matrix. The inverse of $\mathbf{X}$ is denoted as $\mathbf{X}^{-1}$. Superscripts are used as indexes of discrete time instants.

\subsection{System Model}

We consider a universal frequency reuse cellular system consisting of base stations (NodeBs or e-NodeBs) with one antenna each ${ }^{1}$. Uplink CoMP is considered here. The difference in terms of delay between uplink and downlink is that there is a larger delay before the CSI is transmitted over backhaul networks in the downlink case. Therefore it is straightforward to extend our work to the downlink scenario.

\subsubsection{System Topology}

In traditional cellular network architecture, a BS just processes its own signal and the signal from the UEs severed by other BSs are considered as noise. Here we consider a cooperating hierarchical system architecture as shown in Fig. 3.1. All the base stations are connected to a base station controller (BSC), which is the central controller that decides how the base stations cooperate. When a cooperating pattern has been decided, the uplink signals in a cluster will be processed by the super base station formed by BSs in a cluster or a central controller. Compared with traditional architecture, there are two unique parameters that are important to the system throughput, i.e., cooperating pattern and rate allocation. Cooperating pattern is inherently an interference management scheme [93], which dictates how the UEs in different clusters interfere with each other. Given a cooperating pattern, another issue we need to address is how much data rate the UEs can transmit. If a

\footnotetext{
${ }^{1}$ The proposed scheme can be easily extended to the case where BSs and/or mobile users have multiple antennas.
} 


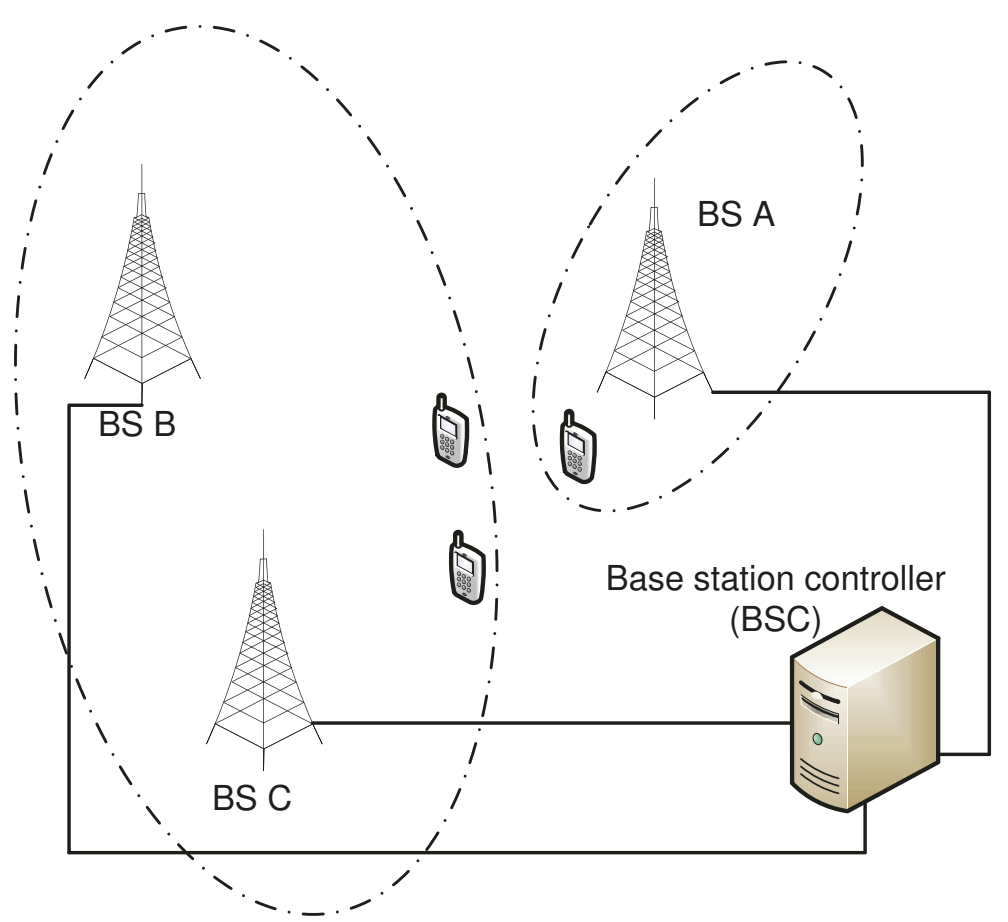

Figure 3.1: Multi-cell multiple users scenario.

rate cannot be supported by the fading channel realization, there is an outage.

The set of BSs in the system is denoted as $\mathcal{B}=\{1,2, \ldots, B\}$. With CoMP, we dynamically change the cooperating pattern of the BSs. Denote the BSs inside a cooperating set as $\omega_{n}$. We assume the maximum number of cooperating BSs is $N_{C}$, namely, the cardinality of each $\omega_{n} \in \Omega$ is no more than $N_{C}$. A cooperating pattern $\Omega$ is a partition of $\mathcal{B}$, i.e.,

$$
\Omega=\left\{\omega_{n} \subseteq \mathcal{B}: \omega_{n} \cap \omega_{n^{\prime}}=\emptyset, \forall n \neq n^{\prime}, \cup_{\omega_{n} \in \Omega} \omega_{n}=\mathcal{B}\right\}
$$

For instance, in Fig. 3.1, BS $B$ and BS $C$ form a cluster; while BS $A$ itself is another cluster. Intuitively, the larger the cooperating set, the better the performance we can expect. However, due to the overhead and imperfectness of CSI, only a limited 
number of BSs should cooperate.

\subsubsection{Finite State Markov Chain Channel Model}

Finite state Markov chain (FSMC) models have been widely accepted in the literature as an effective approach to characterize wireless channels [87], including satellite channels [88], indoor channels [89], Rayleigh fading channel [90], Ricean fading channel [91] and Nakagami fading channel [92]. In this chapter, we use homogeneous FSMC of order one to model the wireless channel in CoMP cellular networks.

For a channel from UE $u$ to BS $b$, to quantize the continuous channel states into discrete CSI, we use the thresholds denoted as $\mathcal{V}_{b, u}=\left\{V_{0}, \ldots, V_{M-1}, V_{M}\right\}$, in which $0=V_{0}<\ldots<V_{M-1}<V_{M}=\infty$. Accordingly, the discrete channel states are denoted as $\mathcal{I}_{b, u}=\{0, \ldots, M-1\}$. In time slot $t$, the channel state from a mobile user to a BS $b$ is in the state $m \in \mathcal{I}_{b, u}$ if the continuous channel gain $h_{b, u}^{t}$ satisfies $V_{m-1} \leq h_{b, u}^{t}<V_{m}$

The dynamics of a FSMC is captured by the initial state distribution and the state transition probability matrix. The transition probability between two states $i, j \in \mathcal{I}_{b, u}$ is denoted as $\operatorname{Pr}\left(I_{b, u}^{t+1}=j \mid I_{b, u}^{t}=i\right)$. Note that the selection of quantization levels has significant effect on the transition matrix. A detail discussion on this is presented in Subsection 3.3.1.

\subsubsection{Physical Layer}

Consider a cooperating set $\omega$ whose cardinality is $|\omega|=K$. Signals for UEs served by BSs in $\omega$ can be decoded without interfering with each other; while the UEs served by the non-cooperating BSs, $\mathcal{B}-\omega$, are interferers to $\omega$. For the sake of convenience, we number the BSs in $\omega$ from 1 to $K$, and BSs in $\mathcal{B}-\omega$ from $K+1$ to $B$. Denote the complex channel gain from a user equipment served by BS $i$ to the antennas of 
all the BSs in $\omega$ as $\mathbf{h}_{i} \in \mathbb{C}^{K \times 1}, i=1, \ldots, K, K+1, \ldots, B$.

We assume that each user equipment is allocated with the same transmission power $P$. If the complex data symbols of user equipments served by cluster $\omega$ are $\left[\begin{array}{lll}x_{1} & \ldots & x_{K}\end{array}\right]$, and the data symbols of user equipments served by the other BSs are $\left[\begin{array}{lll}x_{K+1} & \ldots & x_{B}\end{array}\right]$, the received signal of the antennas of cluster $\omega$ is given by

$$
\mathbf{y}=\sqrt{P} \sum_{l=1}^{K} \mathbf{h}_{l} x_{l}+\sqrt{P} \sum_{l^{\prime}=K+1}^{B} \mathbf{h}_{l^{\prime}} x_{l^{\prime}}+\mathbf{n},
$$

where $\mathbf{n}$ is a vector of independent complex circularly symmetric additive Gaussian noise with each element $n \sim \mathcal{C N}\left(0, N_{0}\right)$. In the above signal level representation, the first term is the useful signal inside $\omega$, while the second term is interference signal from $\mathcal{B}-\omega$.

With Minimum Mean Square Error - Successive Interference Cancellation (MMSESIC) receiver [93, Ch. 10.1], the multiple access channel capacity can be achieved. If the decoding order is fixed, the data rates are given as follows:

$$
\begin{aligned}
& R_{K}=\log \left(1+\frac{P\left\|\mathbf{h}_{K}\right\|^{2}}{N_{K}}\right) \\
& R_{K-1}=\log \left(1+P \mathbf{h}_{K-1}^{T}\left(N_{K-1} \mathbf{I}_{K}+P \mathbf{h}_{K} \mathbf{h}_{K}^{*}\right)^{-1} \mathbf{h}_{K-1}\right) \\
& \quad \ldots \\
& R_{2}=\log \left(1+P \mathbf{h}_{2}^{T}\left(N_{2} \mathbf{I}_{K}+\sum_{l=3}^{K} P \mathbf{h}_{l} \mathbf{h}_{l}^{*}\right)^{-1} \mathbf{h}_{2}\right) \\
& R_{1}=\log \left(1+P \mathbf{h}_{1}^{T}\left(N_{1} \mathbf{I}_{K}+\sum_{l=2}^{K} P \mathbf{h}_{l} \mathbf{h}_{l}^{*}\right)^{-1} \mathbf{h}_{1}\right)
\end{aligned}
$$

in which $N_{l}, l=1,2, \ldots, K$ are the AWGN noise accounting for the receiver noise $N_{0}$ 
and the interference from outside $\omega$. Specifically, the total noise at the $l^{\text {th }}$ antenna is

$$
N_{l}=N_{0}+P \sum_{l^{\prime}=K+1}^{B}\left|\mathbf{h}_{l^{\prime}}\right|^{2} .
$$

Therefore, given a clustering scheme $\Omega$, the sum rate of the whole system is

$$
R(\Omega)=\sum_{\omega \in \Omega} \sum_{b \in \omega} R_{b}(\omega)
$$

Note that, even in the work, the data rates are computed based on MMSE-SIC, the framework we develop in the following is still applicable to other physical layer techniques such as beam-forming, since the only difference is the various selections of objective functions.

\subsection{Delayed CSI Issue in Uplink CoMP}

In this section, we focus on demonstrating the delay in CSI and its effect. The analysis is based on modeling the channels as finite state Markov chains. Firstly, we introduce the definition of system state in our formulation. Then a case study of the delay in uplink CoMP is shown. At the end, we analyze the performance of the existing cooperating scheme that makes the decisions based merely on current CSI observation. Such analysis shows some insights of the fundamental mechanisms provided by the Markov channel modeling.

\subsubsection{System State Based on FSMC}

Recall that, in Subsection 3.2.2, we characterize the complex channel gain from the transmit antenna to the receive antenna as a Markov chain with $M$ states. The quantization and partitioning of the continuous value channel states into discrete ones 
is a very important step in channel modeling. Some approaches have been proposed in the literature. In [90], an equal probability method is used such that the resulted stationary probability of all states are the same. The authors of [94] partition the channel values in a way that the expected sojourn time of all the discrete states are the same. Recently a so-called Adjacent Transition method is proposed in [95] that aims to reduce the assumptions needed in the previous partitioning methods. To minimize the distortion caused in quantization, we adopt the Lloyd-Max approach [96] that results in minimum quantization mean square error (MSE) [97, 98]. LloydMax algorithm iteratively computes the thresholds and the representation values. It first finds an arbitrary initial set of representation points, then sets the thresholds $V_{1}, \ldots, V_{M-1}$ to be the means of the two adjacent representation values. The new representation values are equal to the conditional mean of the input channel sample given it is between the thresholds, which minimizes the mean square error. The iteration continues until the mean square error is minimized [96].

In a CoMP system with $B$ base stations and one UE for each cell, the state space of the networked-MDP model is the Cartesian product of $B^{2}$ FSMCs. Namely, $\mathcal{S}=\mathcal{I}_{1,1} \times \mathcal{I}_{2,1} \times \ldots \times \mathcal{I}_{b, u} \times \ldots \times \mathcal{I}_{B, B}$, in which $\mathcal{I}_{b, u}$ is the FSMC from a transmit antenna of UE $u$ to a receive antenna of BS $b$, and the size of the state space is $M^{B^{2}}$.

Denote the system state at slot $t$ as $S^{t}$, decided by the $B^{2}$ channels $\left[I_{1,1}^{t}, I_{2,1}^{t}, \ldots, I_{b, u}^{t}, \ldots, I_{B, B}^{t}\right]$. At the next slot, the system state is $S^{t+1}$, decided by $\left[I_{1,1}^{t+1}, I_{2,1}^{t+1}, \ldots, I_{b, u}^{t+1}, \ldots, I_{B, B}^{t+1}\right]$. Then we can define the state-transition function $T$. According to the networked-MDP model, the probability of the next system state is decided by the current system state and the actions taken by all the subsystems. As a special case of the network-MDP, here the system state, namely the channel state, is independent of the actions of BSs. Besides, since in multi-cell systems, the transmit and receive antennas are separated with large distance, we can assume that 
the propagation of each signal is independent of each other. So, provided that the current state is $s$, the probability for the next system state will be $s^{\prime}$ is given by

$$
\begin{aligned}
T\left(s, s^{\prime}\right) & =\operatorname{Pr}\left(S^{t+1}=s^{\prime} \mid S^{t}=s\right) \\
& =\prod_{b=1, u=1}^{b=B, u=B} \operatorname{Pr}\left(I_{b, u}^{t+1} \mid I_{b, u}^{t}\right) .
\end{aligned}
$$

Accordingly, the $d$ steps transition probability is given by $T^{d}$. Conceptually, the size of the matrix $T$ is $M^{B^{2}}$-by- $M^{B^{2}}$. However, as a matter of fact, the matrix is a special sparse matrix with non-zero elements lying along the diagonal. The data structure to store such a sparse matrix is usually a linked list, and to operate such sparse matrix is quite convenient [99].

For observations, denote the observation space as $\mathcal{O}$, we can easily see that $\mathcal{O}=$ $\mathcal{S}$. In the following discussion, we use $o \in \mathcal{O}$ and $s \in \mathcal{S}$ to represent a particular realization of the observation and that of the state, respectively. When the temporal relationship is concerned, we use superscripts, say $t$, to differentiate different states or observations.

\subsubsection{Delay in CSI Sharing}

To see how delay comes into CoMP, we first consider a cellular network architecture with a central controller, for instance, the one shown in Fig. 3.1. There are links connecting BSs and BSC, BSs can signal each other via such a backhaul network. There is also another type of architectures. In fact, hierarchical structures are assumed in some related work such as [12]. In [12], there is a medium level controller called cluster manager that is a BS appointed by BSC, and the signal processing can happen at cluster manager. For the sake of simplicity, we only consider the case where the 
Channel Fading
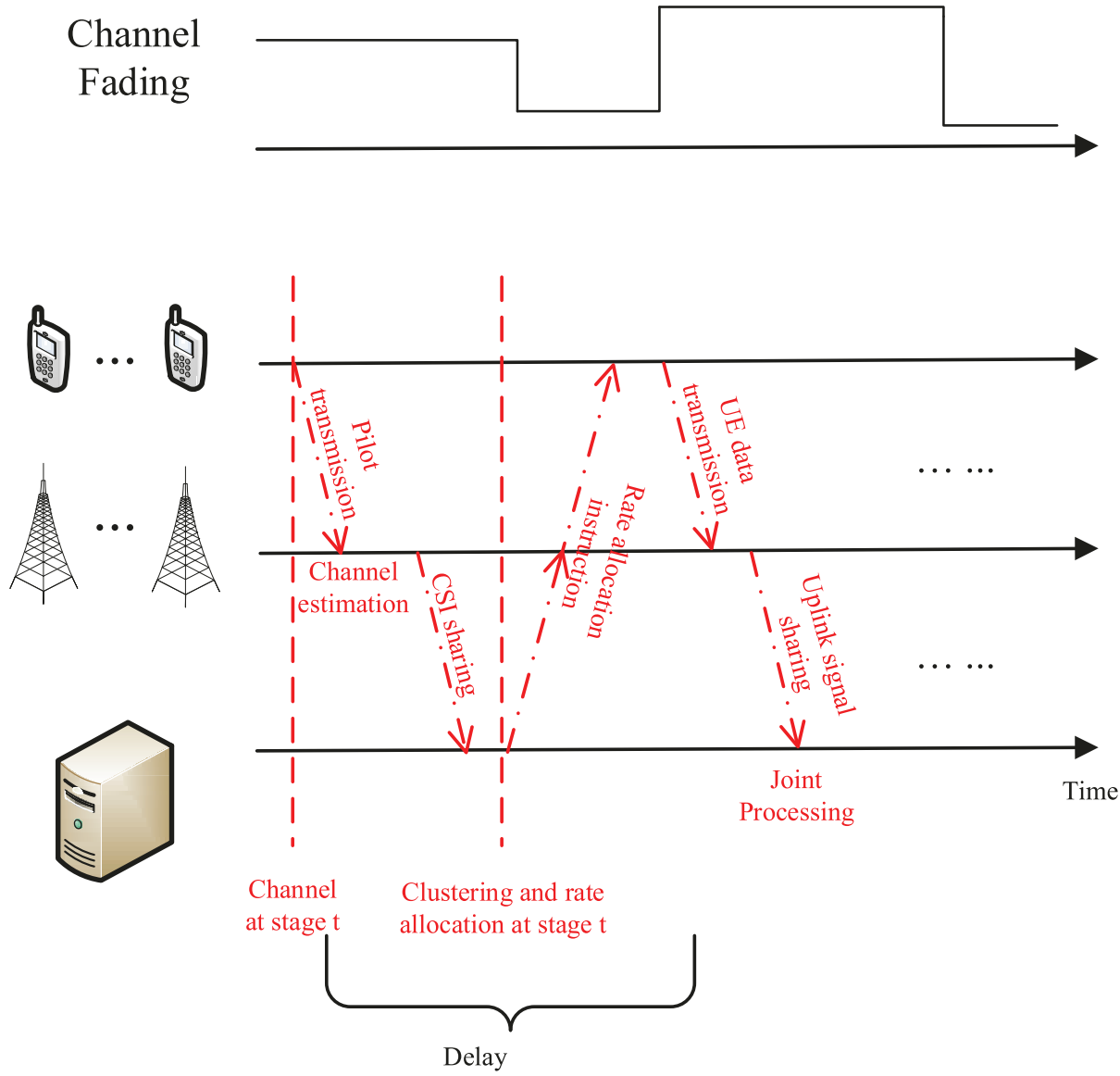

Figure 3.2: Delay in multi-cell multi-user scenario.

central controller in charge of decision making and signal processing. In such an architecture, there are three layers in the systems: UEs, BSs, BSC. Nevertheless, both architectures can be supported by the proposed scheme in this chapter.

Fig. 3.2 shows the processing procedure, the CSI is first obtained via pilot signals received at base stations. After channel estimation, the CSI will be transmitted over backhaul networks to BSC. At BSC, a decision about how the BSs cooperate and the rates at which UEs can transmit are decided after obtaining CSI. Then, the user data is transmitted. The user signals are transmitted from UEs to BSs, and then are propagated over the backhaul networks. At the moment of decision making, the available CSI is outdated. While after the decision making, the channel dynamics 
are actually happening in the future from the point of view of decision making. For example, at decision stage $t$ in Fig. 3.2, the clustering and rate allocation are decided after receiving the CSI representing the channel some moments ago; after that, the actual packet transmissions happen in maybe another channel realization. However, in a nutshell, we can sum all the delay into one parameter as shown in Fig. 3.2.

The above analysis is for the uplink case. For downlink, the principle is the same except that the parameter of delay could be larger than in the uplink case. The reasons are as follows. If the network is a FDD system, the UEs need to feedback the channel measurement to the BSs, which leads to larger delay before decision making; if it is a TDD system, the measurement occurred in the previous uplink data frame, hence the delay is larger in channel estimation. So the effect of the inaccuracy of the channel state information is more severe in downlink than in uplink.

\subsubsection{Analysis of CoMP Based Merely on Outdated CSI}

To see how the delay impacts the performance, we derive the outage probability as a function of delay in CSI in a scheme where the decisions are made only based on current observation. The outage probability will ultimately determine the throughput since the throughput is up to the rate allocations and outage probability. To simplify the derivation, we only consider one UE since the system-wide throughput is the sum of all UEs' throughput.

Proposition 3.3.1. For a UE, provided that the CSI delay is $d$, and that the current observation is $o \in \mathcal{O}$, its outage probability is

$$
\operatorname{Pr}(\text { outage })=\sum_{o \in \mathcal{O}} \operatorname{Pr}(o) \sum_{s^{\prime} \in \mathcal{S}: R(o)>C\left(s^{\prime}\right)} T^{d}\left(o, s^{\prime}\right)
$$

in which $\operatorname{Pr}(o)$ is the stationary state distribution, $s^{\prime}$ and $C\left(s^{\prime}\right)$ are the actual channel 
state and its channel capacity, $T^{d}$ is the d-step transition matrix, and $R(o)$ is the rate allocation for the UE.

Proof. Given a clustering action and rate allocation, the outage probability is decided by the probability measure where the channel capacity is smaller than the rate allocation. In existing schemes, the rate allocation for the UE is a function of the current observation, denoted as $R(o)$. We assume that the rate allocation is the same as the channel capacity as if the actual channel state is $o$. Denote the actual channel state is $s^{\prime}$ and its corresponding channel capacity is $C\left(s^{\prime}\right)$. Conceptually, the outage probability is the sum of the probabilities of the states, whose the channel capacity is smaller than rate allocation $R(o)$. Namely,

$$
\begin{aligned}
\operatorname{Pr}(\text { outage } \mid o) & =\sum_{s^{\prime}: R(o)>C\left(s^{\prime}\right)} \operatorname{Pr}\left(s^{\prime} \mid o\right) \\
& =\sum_{s^{\prime}: R(o)>C\left(s^{\prime}\right)} T^{d}\left(o, s^{\prime}\right) .
\end{aligned}
$$

Hence, given the distribution of observation $\operatorname{Pr}(o)$, the average outage probability is as follows,

$$
\begin{aligned}
\operatorname{Pr}(\text { outage }) & =\sum_{o \in \mathcal{O}} \operatorname{Pr}(\text { o }) \operatorname{Pr}(\text { outage } \mid o) \\
& =\sum_{o \in \mathcal{O}} \operatorname{Pr}(o) \sum_{s^{\prime}: R(o)>C\left(s^{\prime}\right)} T^{d}\left(o, s^{\prime}\right) .
\end{aligned}
$$

The above equation means the outage probability is dictated by the distribution of the states and the $d$-step transition matrix.

As an example, Fig. 3.3 shows the raw outage probabilities of a system with three users. We can see that the outage probability increases as the CSI gets more and more outdated. To see the effect of delay, schemes such as error margins and link layer retransmission are not used in obtaining the numerical data. With these 


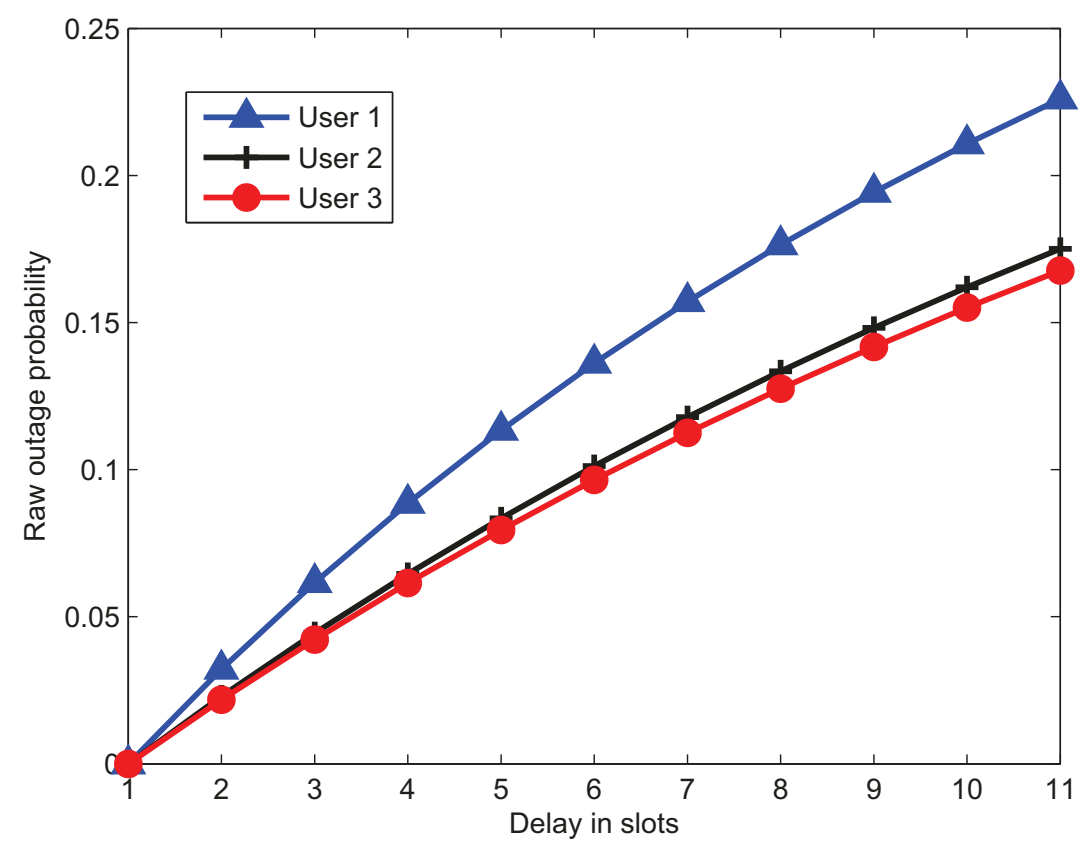

Figure 3.3: Raw outage probability of an uplink CoMP system consisting of three users.

techniques, we can expect to see smaller outage probabilities.

In the following, we will see how the outage probability converges as delay goes to infinity. Assume that the FSMSs are irreducible, aperiodic, and all states are ergodic. The stationary distribution $\pi_{s}=\operatorname{Pr}(S=s)$ and the probability transition matrix $T$ have the following relationship [100, pp. 448],

$$
\pi(s)=\lim _{d \rightarrow \infty} T^{d}\left(s, s^{\prime}\right)
$$

Such stationary distribution can also apply to the observation space. Therefore, as the delay goes to infinity, the outage performance of CoMP based only on outdated CSI is dictated by the stationary distribution. In particular we have

$$
\lim _{d \rightarrow \infty} \operatorname{Pr}(\text { outage })=\sum_{o \in \mathcal{O}} \pi(o) \sum_{s^{\prime} \in \mathcal{S}: R(o)>C\left(s^{\prime}\right)} \pi\left(s^{\prime}\right)
$$


Furthermore, the convergence rate of the transition matrix towards stationary distribution is dictated by the second largest eigenvalue of the transition matrix [101].

\subsection{Optimal Clustering and Rate Allocation for CoMP Systems with Delayed CSI}

In this work, we assume that the power allocated to each user is the same, so that we only need to consider the optimal clustering and rate allocation problem. Note that, it is straightforward to adapt this framework to support power allocation by taking the possible power allocation scheme into the action space defined in the following.

\subsubsection{Actions and Reward Functions}

At slot $t$, BSC selects the cooperating BSs and allocates the rate for UEs. Denote the clustering action at slot $t$ as $\Omega^{t}$, which is a partition of the whole network defined in (3.1), and the rate allocations as a vector with $B$ elements, $\mathbf{R}^{t}=\left[R_{1}^{t} \ldots R_{B}^{t}\right]$. The overall action is $a^{t} \triangleq\left\{\Omega^{t}, \mathbf{R}^{t}\right\} \in \mathcal{A}$, where $\mathcal{A}$ is the set of available actions.

At the end of each slot, the data transmissions are either successful or failed due to outage. In the network-MDP terminology, the multi-cell system will receive an immediate reward. For a UE $u, u=1,2, \ldots, B$, denote the instantaneous throughput as $\zeta_{u}\left(S^{t}, a^{t}\right)$, which is a random variable decided by the system state $S^{t}$ and the action

taken $a^{t}$. The reward function is a sum of the throughput of all UEs, $\sum_{u=1}^{u=B} \zeta_{u}\left(S^{t}, a^{t}\right)$. The objective of the uplink CoMP is to maximize the average cumulative rate over $h$ slots, i.e., the physical layer throughput. Such objective function is the averaged expected cumulative rewards over $h$ horizons in network-MDP. Namely, 


$$
\underset{a^{t}, t=t_{0}, t_{0}+1, \ldots, t_{0}+h-1}{\operatorname{maximize}} \mathbb{E}\left[\frac{1}{h} \sum_{t=t_{0}}^{t=t_{0}+h-1} \sum_{u=1}^{u=B} \zeta_{u}\left(S^{t}, a^{t}\right)\right] .
$$

For a particular user, say $u$, if the current channel capacity is less than the transmission rate allocated, there is an outage such that the resulted transmission rate is 0 ; otherwise, the resulted transmission rate is equal to the allocated rate. In particular, we have

$$
\zeta_{u}\left(S^{t}, a^{t}\right)= \begin{cases}0, & \text { if } R_{u}^{t}>C_{u}\left(S^{t}, a^{t}\right) \\ R_{u}^{t}, & \text { otherwise }\end{cases}
$$

where $C_{u}\left(S^{t}\right)$ is the channel capacity for a UE $u$. Provided the action $a^{t}$, denote the outage probability of user $u$ as $\operatorname{Pr}_{u}\left(\right.$ outage $\left.\mid a^{t}\right)$. Hence the actual successful transmission rate in a slot $t$ also depends on the outage probability. Namely,

$$
\mathbb{E}\left[\zeta_{u}\left(S^{t}, a^{t}\right)\right]=R_{u}^{t} \cdot\left[1-\operatorname{Pr}_{u}\left(\text { outage } \mid a^{t}\right)\right]
$$

For a network-MDP, the concept of a solution is a policy defined as a sequence of actions that maximize the rewards or minimize the cost. In the following, we discuss the optimal policy for the uplink CoMP problem.

\subsection{Belief State and Greedy Policy}

To solve the stochastic optimization problem defined in (3.9), the general approach is to use dynamic programming over belief state, which is the sufficient statistic of the previous action and observation history [74]. A belief state $\mathbf{b}^{t}$ at slot $t$ is defined as a probability distribution of the state space. Accordingly, the probability that the 


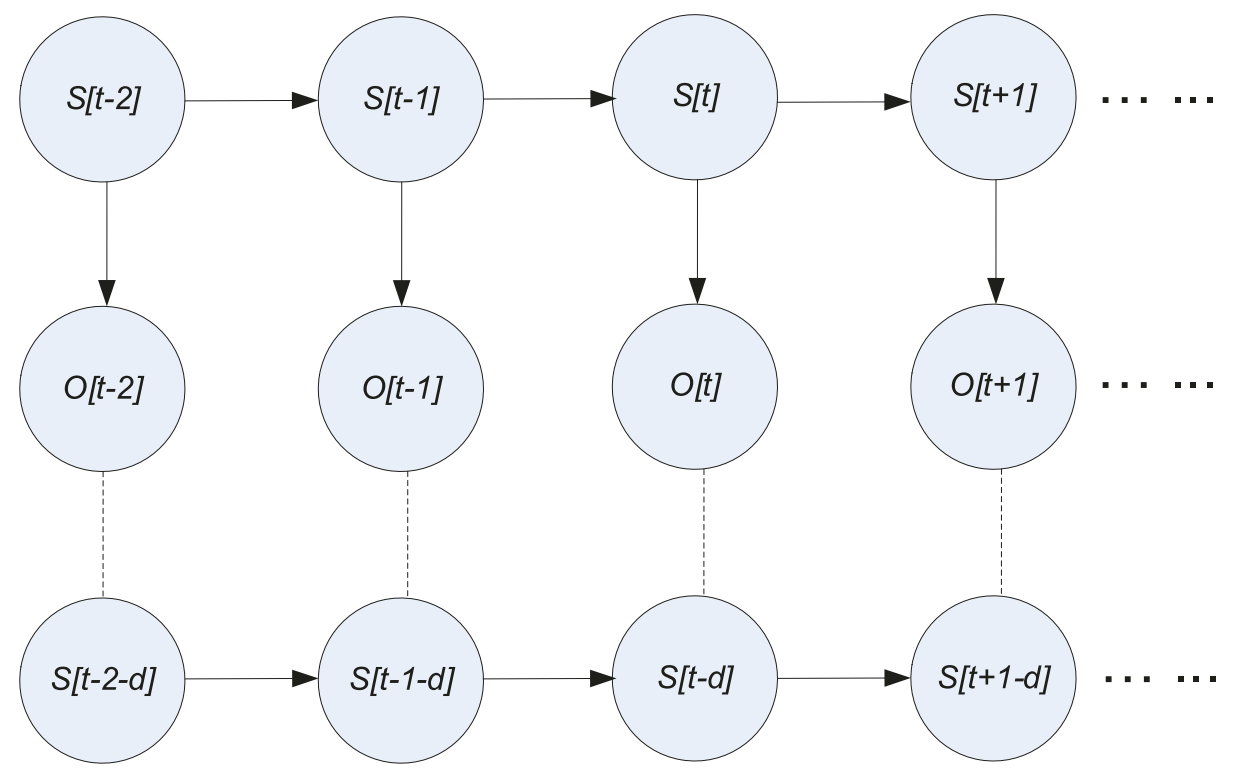

Figure 3.4: $d$ steps delayed observation.

state at slot $t$ is $s^{t}$ is given by the corresponding element in $\mathbf{b}^{t}$, denoted as $b\left(s^{t}\right)$. In this section, we first develop the belief states with known and unknown delay steps. Then we discuss the optimal policy for the decision making problem.

\subsubsection{Belief State with Known Delay Steps}

To illustrate the relation between observations and actual states, we use a directed graphical model shown in Fig. 3.4. In the figure, each node represents a random variable, the arrow presents a conditional probability, and the dash line represents that the two random variables are equivalent. The first row of the random variables are the states of the system, which form a first-order Markov chain. The second row is a sequence of observations. The arrow between neighboring states is the statetransition function, while the arrow between a particular state and a corresponding observation is the observation function.

With techniques such as timestamping, we can know the number of delay steps $d$. With such assumption, the observation is just the actual state delayed by $d$ steps. 
Namely for the two random variable $O^{t}$ and $S^{t}$, we have $O^{t}=S^{t-d}, t=d+1, \ldots$ Thus we can derive the explicit relation between the current state and observation. The belief state is

$$
\begin{aligned}
b\left(s^{t+1}\right) & =\operatorname{Pr}\left(s^{t+1} \mid o^{t+1}, o^{t}, \ldots\right) \\
& =\operatorname{Pr}\left(s^{t+1} \mid s^{t+1-d}, s^{t-d}, \ldots\right) \\
& =\operatorname{Pr}\left(s^{t+1} \mid s^{t+1-d}\right) \\
& =T^{d}\left(s^{t+1-d}, s^{t+1}\right) .
\end{aligned}
$$

The third equality is given by first order Markov property assumed in the FSMC channel model. And $T^{d}$ is the $d$ steps probability transition matrix.

\subsubsection{Belief State with Unknown Delay Steps}

If it is difficult to get the number of delay steps, we can still derive the belief state as follows. We first introduce Observation Function [74], then show how to derive belief state from the state-transition function $T(\cdot)(4.8)$ and observation function $O(\cdot)$ (4.10). State-transition function and observation function can be obtained via classical algorithms such as Expectation Maximization [102].

\section{Observations and Observation Function}

Assume at slot $t$, the observation of the system is $o \in \mathcal{O}$, which is decided by the observations over $B$ channels. The observation function $O(\cdot)$ essentially depicts the probabilistically relationship between an observation $o \in \mathcal{O}$ and a state $s \in \mathcal{S}$. Formally, observation is also a function of the action taken, however, in our problem here the observation is independent of the action taken, so it is defined as

$$
O(s, o)=\operatorname{Pr}(o \mid s)
$$




\section{Belief Update Based on Bayesian Rule}

Essentially, provided a new observation at time $t+1, o^{t+1}$, the new belief should reflect the likelihood of ending up in new state $s^{t+1}$, the likelihood of observing $o^{t+1}$, and the pervious belief distribution $\mathbf{b}^{t}$. Rigorously, the rule to update the belief state according the previous belief and current observation is based on the Bayesian rule [74] defined as

$$
\begin{aligned}
& b\left(s^{t+1}\right)=P\left(s^{t+1} \mid o^{t+1}, \mathbf{b}^{t}\right) \\
= & \frac{O\left(s^{t+1}, o^{t+1}\right) \sum_{s^{t} \in \mathcal{S}} T\left(s^{t}, s^{t+1}\right) b\left(s^{t}\right)}{\sum_{s^{t+1} \in \mathcal{S}} O\left(s^{t+1}, o^{t+1}\right) \sum_{s^{t} \in \mathcal{S}} T\left(s^{t}, s^{t+1}\right) b\left(s^{t}\right)}
\end{aligned}
$$

The second equality is based on the fact that the channel dynamics is independent of the clustering and rate allocation actions.

\subsubsection{Greedy Policy}

Note that the CoMP system considered here is simpler than the networked-MDP considered in $[1,73]$ because there is only delay between the subsystems(BS) and the controller (BSC). While in general networked-MDP, there is also delay between subsystems. For the optimal clustering problem consider here, since the channels between the UEs and the BSs are independent of each other, the system state in a nutshell is Markovian. So if the system state can be perfectly observed, it is a Markov decision process (MDP). Yet, because the central controller can only see an outdated version of the actual system state, i.e., the system state can only be partially observed, it is a partially observable Markov decision process (POMDP) [74]. General discussion about the equivalence can be found in $[1,73]$. The complexity of the dynamic programming approach to solve the networked-MDP model in (3.9) is PSPACE [74], which is intractable for large state space and horizons. So, we first 
consider a greedy policy that maximizes the expected instantaneous transmission rate in (3.11), i.e., the action to take at slot $t$ is given by

$$
a^{t}=\arg \max _{a^{t} \in \mathcal{A}} \sum_{u=1}^{u=B} R_{u}^{t} \cdot\left(1-\operatorname{Pr}_{u}\left(\text { outage } \mid a^{t}\right)\right) .
$$

We approximate the outage probability with the ease of the Markov chain channel model. Given the rate allocation $R^{t}$ and the clustering action, the outage probability is approximated by

$$
\operatorname{Pr}_{u}\left(\text { outage } \mid a^{t}\right)=\sum_{C_{u}\left(s^{\prime}\right)<R_{u}^{t}} b\left(s^{\prime}\right)
$$

which is the probability of the channel being in the states where the allocated transmission rate $R^{t}$ is greater than the channel capacity $C\left(s^{\prime}\right)$. In the following, we will show that for the uplink CoMP problem the greedy policy defined in (4.19) is the optimal policy. The complexity of the greedy policy will also be briefly discussed.

\section{The Optimality and Complexity of the Greedy Policy}

The optimality of greedy policy is based on the fact that the dynamic of the the channel states is independent of the actions taken. It is in fact straightforward to show. In particular, consider at horizon $h=1$, the optimal action to take is the maximizer of $\mathbb{E}\left[\sum_{u=1}^{u=B} \zeta_{u}\left(S^{1}, a^{1}\right)\right]$, which is obviously the action given by greedy policy to maximize the expected rewards in one step. Assume at horizon $h, h \geq 1$, the optimal policy is the greedy policy given in (4.19). Then at horizon $h+1$,

$$
\begin{aligned}
& \mathbb{E}\left[\sum_{t=0}^{t=h+1} \sum_{u=1}^{u=B} \zeta_{u}\left(S^{t}, a^{t}\right)\right] \\
= & \left\{\mathbb{E}\left[\sum_{t=0}^{t=h} \sum_{u=1}^{u=B} \zeta_{u}\left(S^{t}, a^{t}\right)\right]+\mathbb{E}\left[\sum_{u=1}^{u=B} \zeta_{u}\left(S^{h+1}, a^{h+1}\right)\right]\right\} .
\end{aligned}
$$


So provided the hypothesis that the greedy policy maximizes the first term in the above equation, the action to take to maximize the total expected rewards is the one to maximize the second item, which is equivalent to the case with horizon 1. Therefore, the greedy policy is the optimal policy for the CoMP clustering problem.

The computation cost of the greedy policy in (4.19) lies mainly in computing the expected outage probability (3.16). The number of states is $M^{B^{2}}$, which leads an upper bound of the number of comparison operations to be $M^{B^{2}}$. With $|\mathcal{A}|$ available actions in total, the upper bound of the computation complexity in offline phase is $O\left(M^{B^{2}}|\mathcal{A}|\right)$.

\subsection{Simulation Results and Discussions}

In this section, computer simulations are carried out to evaluate the performance of the proposed clustering and rate allocation scheme. For the time-varying wireless channels, we use the 3GPP-SCME channel model [103], which is widely used in simulations of wireless cellular networks [104]. There are 3 base stations in the system with one antenna for each BS, and the inter-site distance is 800 meters. We fix the number of a cluster to be 2 , so that the number of possible clustering action is 3 . Three existing schemes are used for comparison. In the first one, clustering is based merely on the current observation. Specifically, the BSC selects the best cooperating set and allocates the rates according to the current CSI observation. Error margins are used to have a fair comparison with existing scheme. If there is no error margin, the transmission rate of a UE is the maximum data rate which can be supported by the observed SINR. However, such optimistic decision may cause outage due to inaccuracy in CSI. So, to reduce the outage probability, given the knowledge of the channel SINR, a lower rate is used unless the SINR is greater than the threshold by $\delta$. The usage of error margin increases the reliability of data transmissions. In our 
simulations, the error margins range from 0 to $9.5 \mathrm{~dB}$. The second existing scheme is a universal reuse cellular network without cooperation, in which the interference from neighboring cells are taken as noise. The third existing scheme is the one based on channel prediction using auto- regressive (AR) model-based techniques [22]. It predicts the current channel state information based on the history of outdated CSI, then a clustering and rate allocation decision is made based on the new channel prediction. The order and the size of window in prediction are 2 and 5 , respectively.

Table 3.1: Simulation Parameters for Uplink CoMP

\begin{tabular}{|c||c|}
\hline Parameter & Value \\
\hline Carrier frequency & $2110 \mathrm{Mhz}$ \\
Base station antenna height & $24 \mathrm{~m}$ \\
User equipment antenna height & $0.5 \mathrm{~m}$ \\
Number multipath components & 6 \\
Sampling duration & $1 / 500000 \mathrm{sec}$ \\
Normalized Doppler shift & from 0.01 to 0.06 \\
Pathloss & $30.18+$ \\
Signal bandwith & $26 * \log 10($ distance $)$ \\
Receiver noise power densisty & $15 \mathrm{KHz}$ \\
UE transmit power & $20 \mathrm{dBm}$ \\
\hline
\end{tabular}

The parameters used in the simulations are given in Table 4.1. In the table, the fast fading dynamics is characterized by normalized Doppler shift, which is defined as the Doppler frequency shift times the slot duration. Since the Doppler frequency shift is approximately the inverse of the coherence time, normalized Doppler shift is the ratio of coherence time over slot duration. For the delay, it is measured in the number of state transition steps. For each state transition step, the physical time duration is $1 \mathrm{~ms}$. The channel state value is each state is obtained via averaging the 


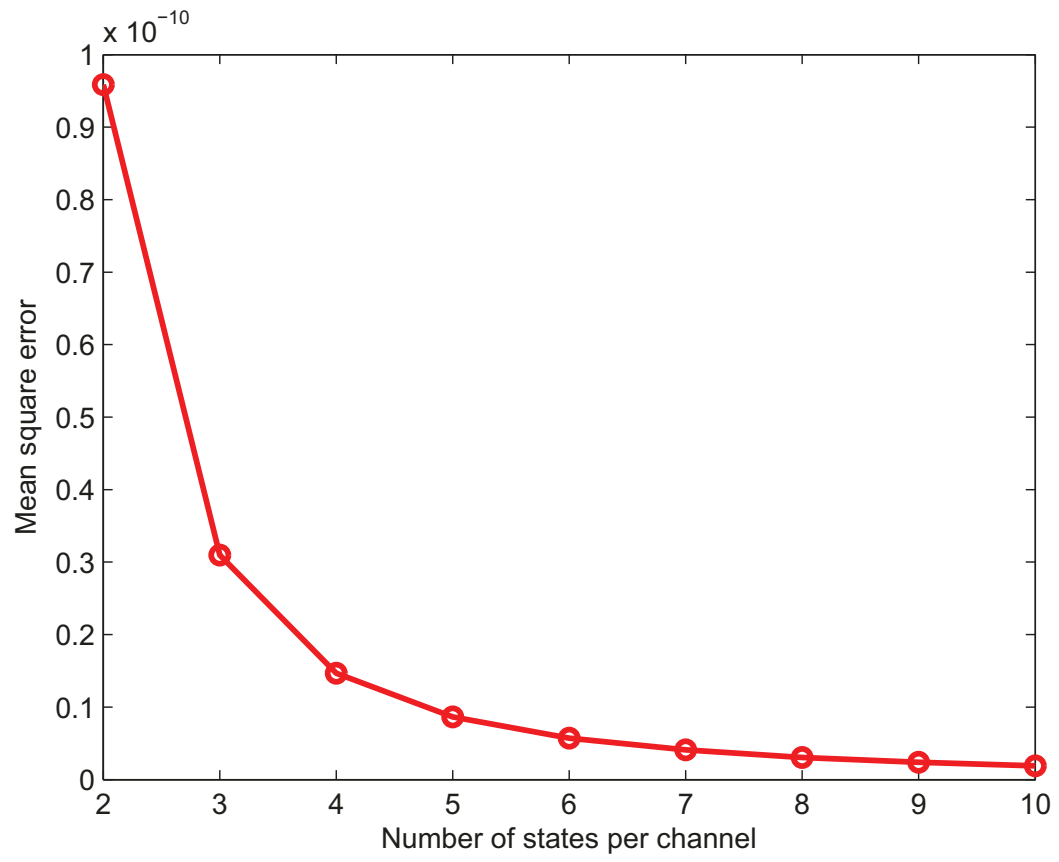

Figure 3.5: Mean square errors versus the number of states per channel in quantization with the Lloyd-Max algorithm.

values the channel state samples.

\subsubsection{Channel Quantization}

In the simulations, we first abstract the stationary state distribution and state transition probability matrix from $2 \times 10^{6}$ channel realizations of fading channel. In quantizing the channel state information, we adopt the Lloyd-Max algorithm [96] that minimizes the mean square error (MSE). An important parameter in quantization is the number of states per channel. As we can see in Fig. 3.5, the MSE decreases exponentially with the increase of the number of states. For the ease of simulations, we quantize the continuous channel values into 2 states. 


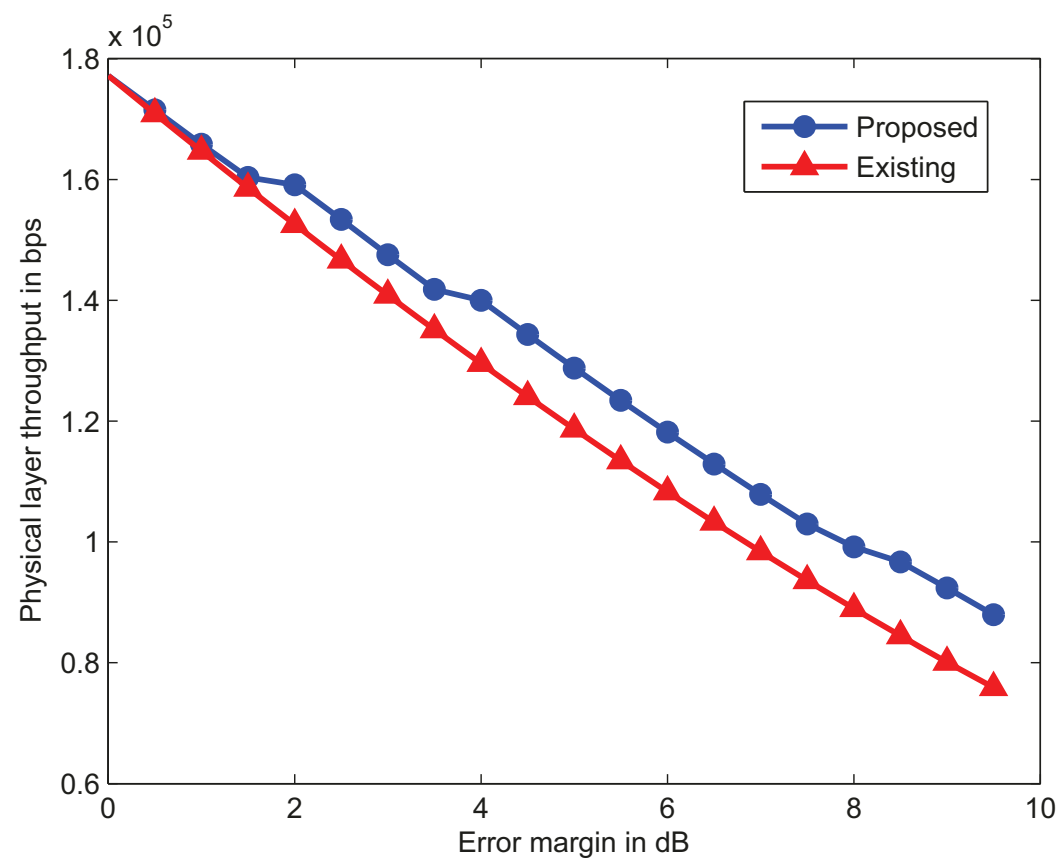

Figure 3.6: The effects of error margins when there is no delay.

\subsubsection{Effects of Error Margin}

The setting of error margin is important for the existing cooperative scheme. For example, Fig. 3.6 shows that both the existing and the proposed scheme deteriorate by using error margin in rate allocation when there is no delay. The normalized Doppler shift is 0.06. With larger error margin, the propose scheme achieves higher data rate. This is because it will be able to choose higher data rate and clustering pattern even the set of rates are constrained by error margin. Fig. 3.7 shows the case where the delay is 3 steps. We can see that, when the error margin is small (e.g., less than $0.5 \mathrm{~dB}$ ), the error margin helps the existing cooperative scheme, where the best performance is reached when the error margin is $0.5 \mathrm{~dB}$. However, error margins tend to be useless for the proposed scheme. So in the following simulations, for the existing scheme we will use the best performance with error margin ranging from 0 dB to $9.5 \mathrm{~dB}$. While for the proposed scheme, we will not use any error margin. 


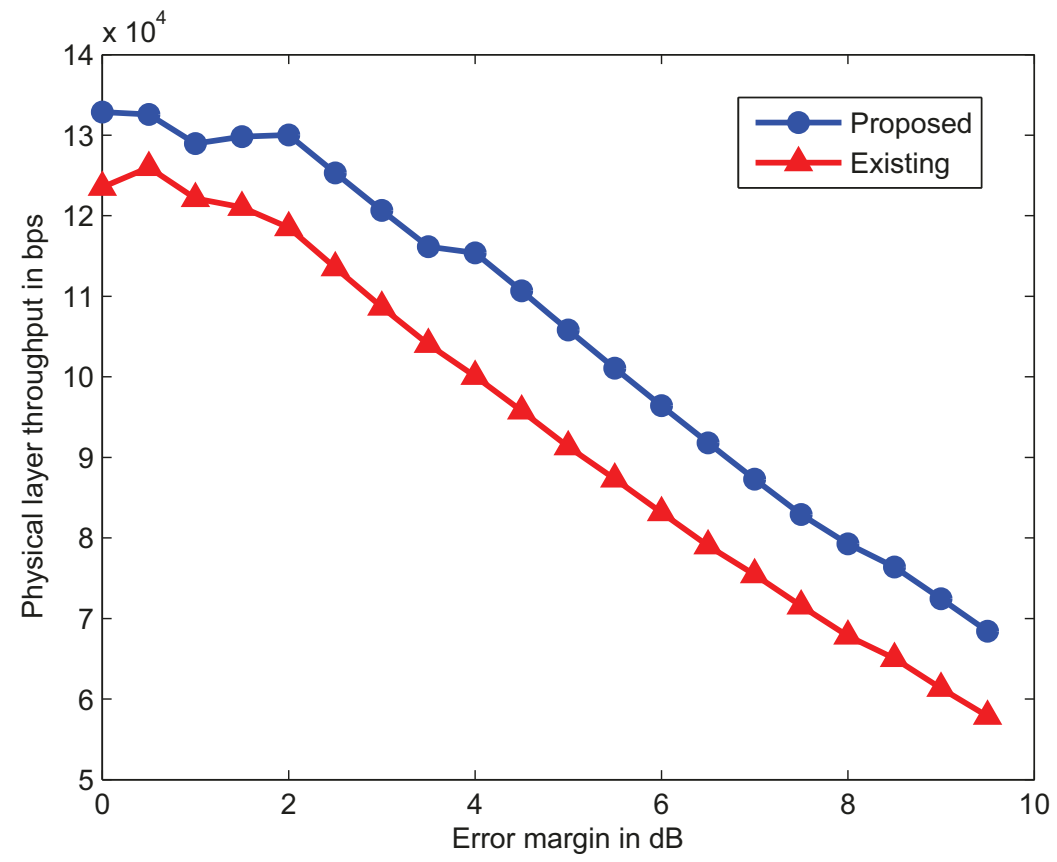

Figure 3.7: The effects of error margins when the delay is 3 steps.

\subsubsection{Effects of Delay}

To study the effects of delay, we conduct simulations in various normalized Doppler shift. Small normalized Doppler shift corresponds to large coherence time and the low mobility scenario. The non-cooperative scheme serves as a baseline. For noncooperative scheme, the delay only comes from the channel estimation. While for the three cooperative schemes, if there is delay, the delay includes the 1 step channel estimation delay plus the backhaul delay.

Fig. 3.8 compares the throughput between existing schemes and the proposed scheme in low mobility case. The physical layer throughput of the three cooperative schemes drops gradually with the increase of delay. The difference between them is minor. This means with low UE mobility, the effect of delay in CSI is not significant. Moreover, it indicates that neither the proposed scheme nor the existing AR-prediction scheme is very capable to reduce such effect. 


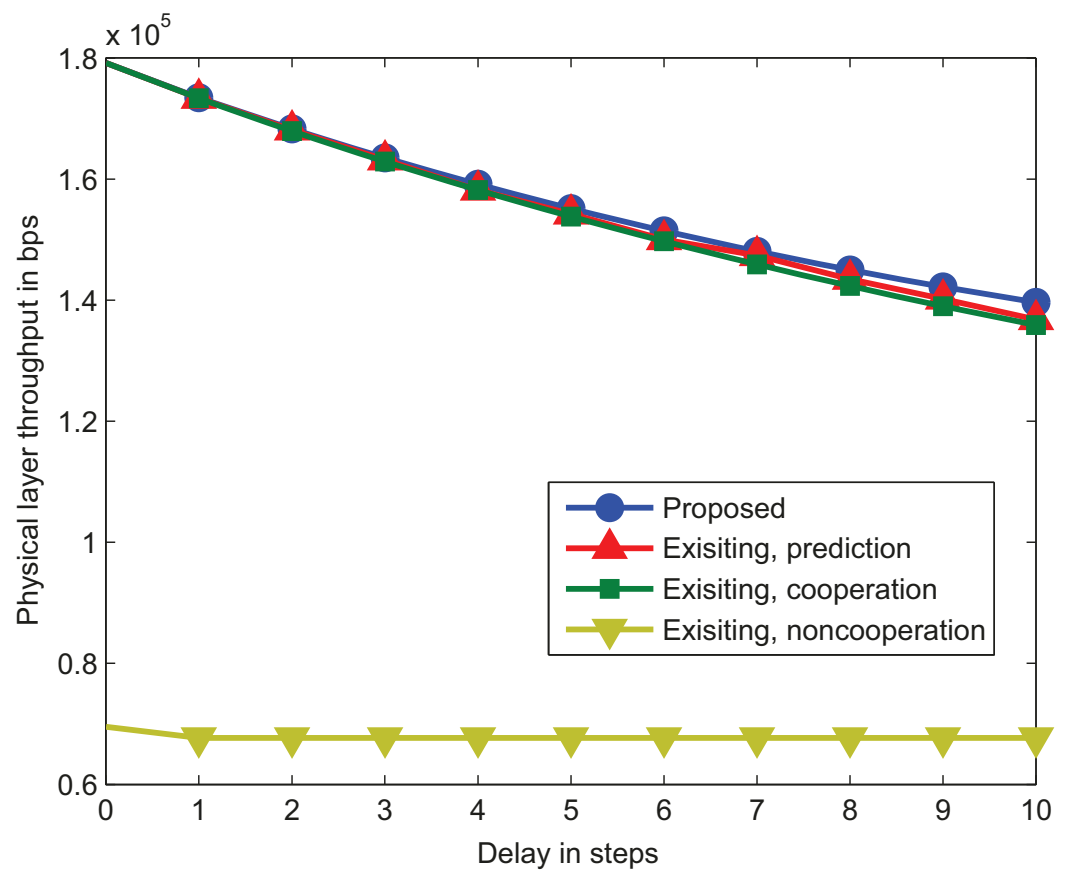

Figure 3.8: The effects of delay on throughput (The normalized Doppler shift is $0.01)$.

With higher UE velocity, namely, large Doppler shift, a higher performance gain can be expected for the proposed scheme. For instance, the results for a normalized Doppler shift of 0.06 are shown in Fig. 3.9. When the delay is smaller than 8 steps, the AR-prediction scheme has some advantage over the existing non-predictive scheme. But the performance of AR-prediction decades dramatically as the delay increases. And its advantage over the existing non-predictive scheme almost diminishes when the delay is large than 9 steps. In contrast, for the proposed scheme, considerable higher throughput is observed when the delay is large. In particular, the proposed scheme outperforms the existing cooperative scheme by more than $30 \%$ when the delay is more than 6 steps. This is largely due to the fact that with large delay, the proposed scheme is able to make use of the stationary distribution of the finite state Markov chains to make better decisions. Another observation in Fig. 3.9 is that the performance of the proposed scheme drops to a constant when the delay is 


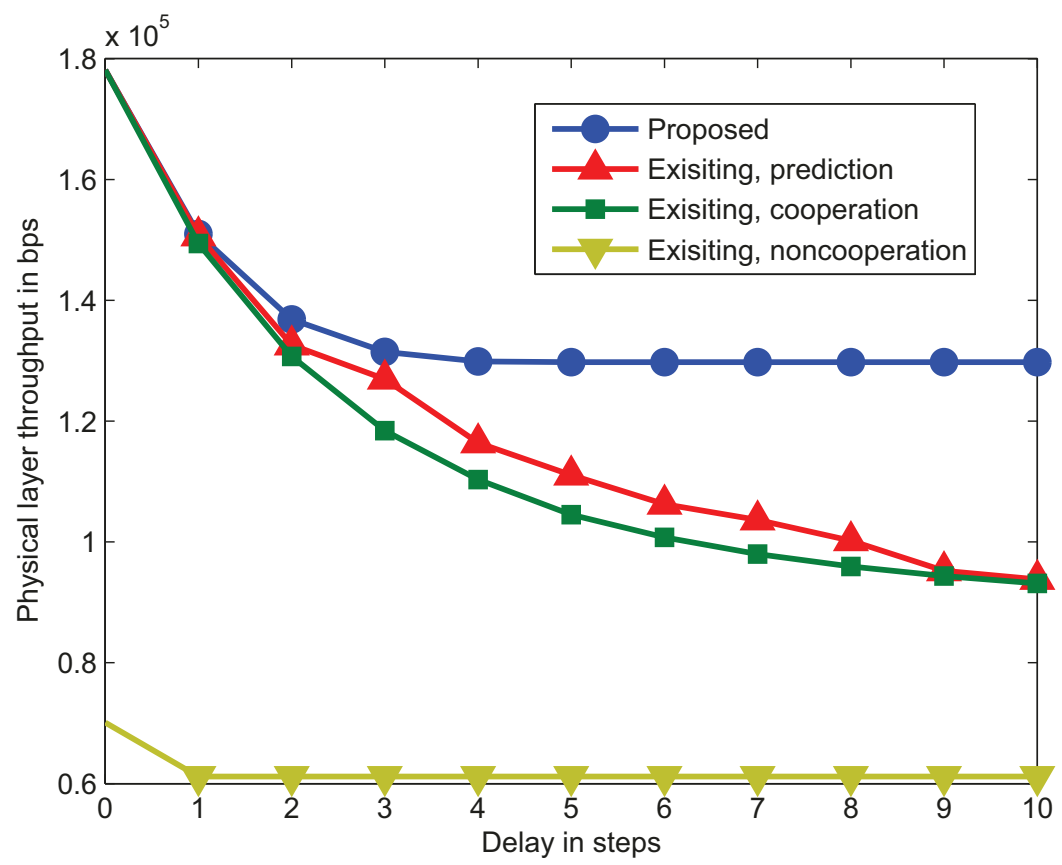

Figure 3.9: The effects of delay on throughput (The normalized Doppler shift is $0.06)$.

greater than 3 . This is mainly because as the increase of delay, the transition matrix converges to the stationary distribution of the wireless channel Markov chain. With merely stationary distribution, the best decisions to maximize (3.9) are the same given different delay values.

\subsubsection{Effects of Mobility}

Another important factor impacting the channel dynamics is the speed of UEs, which is related to Doppler shift. We investigate such effects by ranging the normalized Doppler shift from 0.01 to 0.06 for various delay settings.

With medium delay, for example, 5 steps in Fig. 3.10, the proposed scheme outperforms the prediction scheme. In fact, the existing AR-prediction scheme only has minor gain over the existing cooperative scheme in low mobility cases. Our proposed scheme obtains more throughput than the AR-prediction scheme by around 


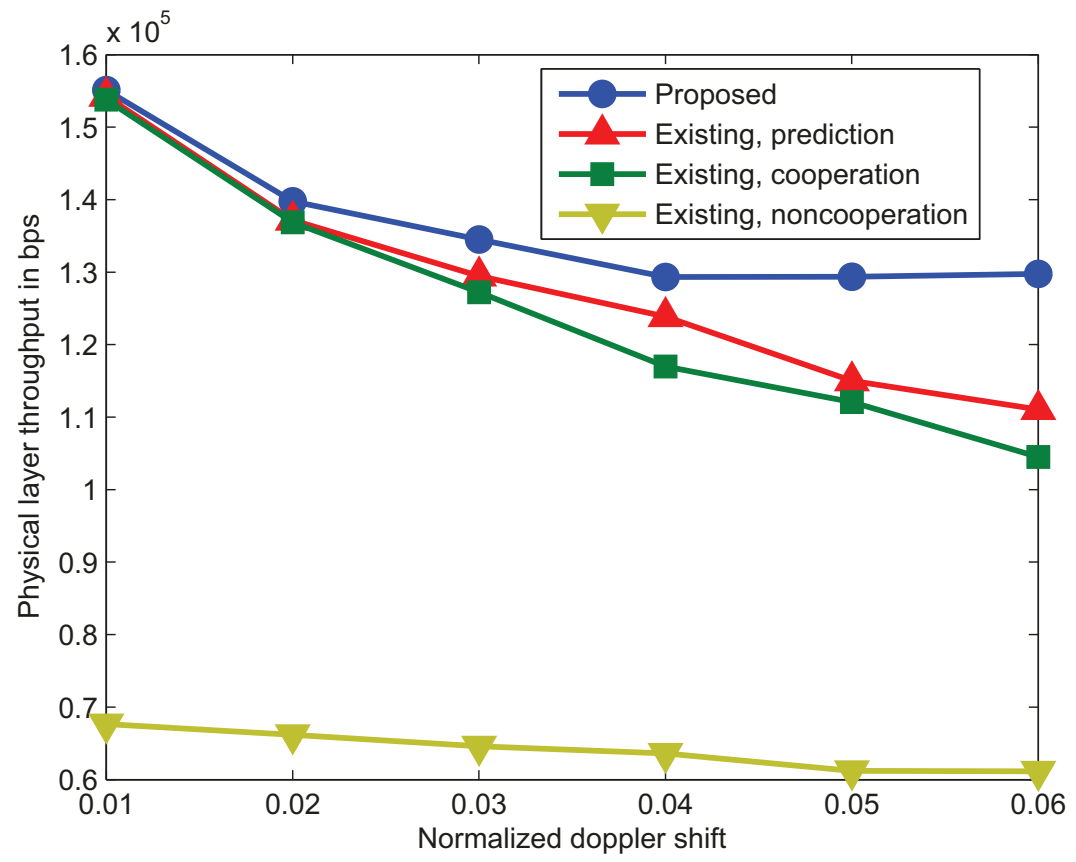

Figure 3.10: The effects of UE mobility speed on throughput when the delay is 5 steps.

$2 \%$ in low mobility case and the advantage increase to around $18 \%$ in high mobility case.

With large delay, e.g., 10 steps in Fig. 3.11, the AR-prediction scheme deteriorates, and it loses the prediction capability when the mobility is high. In contrast, our proposed scheme has significant performance gain and such gain gets larger as the UE mobility increases. Specifically, the proposed scheme achieve about $45 \%$ more data rate than the best existing scheme in high mobility case.

\subsubsection{Discussions}

When the fading process is jointly Gaussian, the auto-regressive based channel prediction is the optimal channel predictor in terms of mean square error. However, its performance decades significantly in high mobility scenario when the delay is large. 


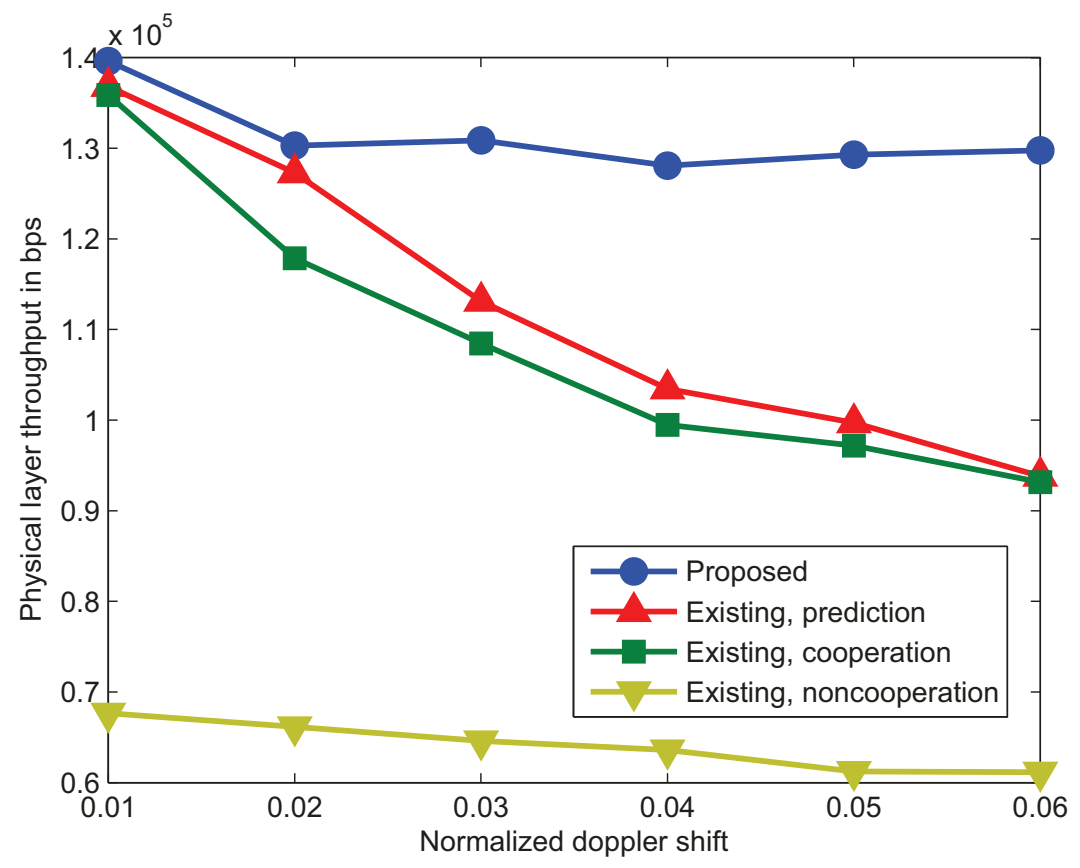

Figure 3.11: The effects of UE mobility speed on throughput when the delay is 10 steps.

Table 3.2: Computation Complexity Comparison

\begin{tabular}{|c|c|c|}
\hline Scheme & Offline phase & Online phase \\
\hline AR-prediction & 0 & $275 \mathrm{~ms}$ per slot \\
Proposed & $8.3 \mathrm{~s}$ & negligible table lookup \\
\hline
\end{tabular}

By contrast, our proposed decision theoretic scheme is not simply predicting the channel state but making the best decision under uncertainty. With large delay and high channel dynamics, the proposed scheme outperforms the AR-prediction scheme.

Furthermore, our proposed scheme has the advantage in terms of computation complexity. The average computation time is shown in Table 3.2. The simulations are conducted on a workstation with an Intel Q8400 CUP and 4GB memory. The version of Matlab used is 2011. In the simulations, we use the built-in functions in Matlab to compute the AR-prediction results. The numbers for the proposed scheme 
is conservative because the codes are not fully optimized, and with the advancement of modern computing technology, such as cloud computing, the offline phase computation can be easily tackled. We can clearly see that the proposed scheme is able to react quickly because the online table lookup time is negligible. By contrast, the computation complexity makes the AR-prediction hardly practical for real-time operations.

\subsection{Chapter Summary}

In this chapter, we investigated the clustering and rate allocation problem for uplink CoMP cellular networks with delayed channel state information. This problem was formulated as a network-MDP with delays. By exploiting the structure of the problem, an optimal policy with low complexity was designed to solve the stochastic optimization problem. Extensive simulations show that the proposed scheme is able to achieve considerable gains compared with existing schemes. 


\section{Chapter 4}

\section{Dynamic Operations of C-RAN in $\mathrm{MCC}$}

\section{Systems}

\subsection{Introduction}

Recently, as a new information technology (IT) paradigm, cloud computing has become one of the hottest topics in both academia and industry. Cloud computing is a model for enabling on-demand access to a shared pool of configurable resources (e.g., servers, storage, applications, services, etc.). The essential characteristics of cloud computing include on-demand self-service, broad network access, resource pooling, rapid elasticity and measured service [105]. Several service models are supported, including cloud software as a service, cloud platform as a service, and cloud infrastructure as service [106]. Cloud computing has attracted significant attention, and several commercial clouds, including Amazon EC2, Microsoft Azure, and Google App Engine, have been providing services to users.

Cloud computing will have profound impacts on the design and operation of wireless networks. On one hand, with recent advances of wireless mobile communication technologies and devices, more and more end users access cloud computing systems via mobile devices, such as smart phones and tablets. The integration of cloud computing 
into the mobile environment enables mobile cloud computing (MCC), which is widely considered as a promising mobile computing paradigm with huge market $[107,108]$. MCC enables offloading the computing power and data storage requirements from mobile devices into the powerful computing platforms in the cloud, bridging the gap between the increasing computing demands and the traditional mobile technologies with limited computing, storage and energy resource in mobile devices [107].

On the other hand, the powerful computing platforms in the cloud can be beneficial to radio access networks (RAN) as well (in addition to mobile end users), which leads to a novel concept of cloud radio access networks (C-RAN) [109-111]. Unlike the existing cellular networks, where computing resources for baseband processing are located at each cell site, in C-RAN, the computing resources are located in a central wireless network cloud with powerful computing platforms. This transition from distributed to centralized infrastructure for baseband processing can have significant benefits: saving the operating expenses due to centralized maintenance; improving network performance due to advanced coordinated signal processing techniques; reducing energy expenditure by exploiting the load variations [109-111].

Although some excellent works have been done to study cloud computing for both end users and access networks, these two important areas have traditionally been addressed separately in the literature. However, as shown in the following, it is necessary to consider these two advanced technologies together to provide better services in next generation wireless networks. Therefore, we jointly study C-RAN in MCC systems so as to improve end-to-end network performance. The motivations behind our work are based on the following observations.

- From end-to-end applications' perspective, both C-RAN and over-the-top (OT$\mathrm{T})$ service provider cloud are parts of the whole system. The experience in 
end-to-end applications (e.g., transmission control protocol (TCP)-based applications) indicates that the optimized performance in one segment of the whole system does not guarantee the end-to-end improvement if the other segment is ignored in the optimization [112].

- It is well known that, while TCP performs relatively well over wired links, its performance degrades over wireless links due to the scarce bandwidth, high bit error rate (BER) and user mobility [113]. In addition, networking has become a bottleneck that has a significant impact on the quality of cloud services [114]. Therefore, the characteristics of C-RAN should be carefully considered in MCC systems.

- Recent studies in cross-layer designs show that optimizing lower layer's performance (e.g., physical layer throughput) does not necessarily benefit quality of service (QoS) at upper layers [31]. From a user's point of view, QoS at upper layers (e.g., TCP throughput) is more important than that at other layers.

To the best of our knowledge, the study of C-RAN in MCC systems for next generation wireless networks has not been addressed in previous works. The distinct features of this work are as follows.

- We consider how to dynamically configure C-RAN to enhance MCC services' performance in a holistic framework. For C-RAN we study the topology configuration and rate allocation problem; for MCC, as a case study, we consider mobile search engine services on top of TCP connections, which has critical requirements on per user response latency. In this work, we optimize the endto-end TCP throughput performance of MCC users in next generation cellular networks. 
- Despite the potential benefits brought by C-RAN, one of the major challenges in C-RAN is that the channel state information (CSI) is inaccurate due to the delay in obtaining and transmitting such information [111]. For instance, in LTE-Advanced, the standard interface for inter-BS communications, $X 2$, is designed to allow a latency of $20 \mathrm{~ms}$ for control plan messages, and the typical value for the latency is expected to be $10 \mathrm{~ms}$ on average [83]. Imperfect CSI has significant impacts on not only C-RAN, but also wireless networks in general [15]. Since it is difficult to solve this problem using traditional information theoretic approach [85], we take a stochastic optimization approach, which has well-developed mechanisms to address the impacts of noisy and delayed CSI. An optimal policy is found based on the particular structure of the topology configuration and rate allocation problem.

- Response latency experienced by cloud users has been recognized as one of the most important performance metrics in cloud computing [35, 115]. Therefore, to improve MCC users' QoS, we model the response latency experienced by each MCC user as a constraint in our formulation.

- We investigate the trade-off between the systematic efficiency and the fairness among MCC users. A parameter is introduced to study such trade-off taking into account the delayed CSI in C-RAN. With this parameter, we re-formulate the problem to maximize the Jain's fairness index in MCC systems.

- Extensive simulations show that, with the emergence of MCC and C-RAN technologies, the design and operation of next generation wireless networks can be significantly affected by cloud computing, and the proposed scheme is capable of achieving substantial performance gains over existing schemes.

The rest of the chapter is structured as follows. Section 5.2 describes the system. 


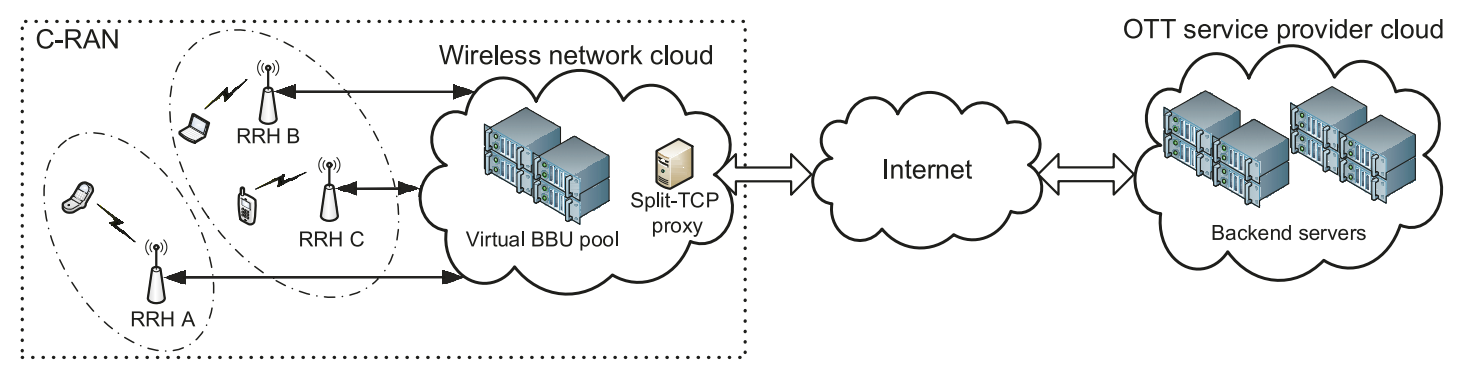

Figure 4.1: A cloud radio access network in the MCC environment.

Section 4.3 discusses the issues caused by delayed CSI in C-RAN. We formulate the problem as a decision theoretic problem in Section 4.4. Fairness and efficiency tradeoff is studied in Section 4.5. Simulation results are discussed in Section 4.6. Finally, we conclude this study in Section 4.7 with future work.

\subsection{System Description}

In this section, we first describe MCC systems and dynamical configurations of CRAN. Then, the physical layer and link layer models for C-RAN are presented.

\subsubsection{Mobile Cloud Computing with Cloud Radio Access Networks}

The system we consider in this chapter is shown in Fig. 4.1, which mainly consists of two sub-systems, i.e., C-RAN and cloud computing. The problem addressed in this work crosses the two sub-systems. The wireless communication mainly happens at the C-RAN, while the processing (e.g., data mining) for the cloud services happens at the backend servers inside OTT service provider cloud.

In C-RAN, the traditional base stations are evolved into a system consisting of remote radio heads (RRHs) distributed in different geographic locations and a baseband process unit (BBU) pool in the wireless cloud $[110,116]$. The RRHs are connected 
to the wireless network cloud via backhaul networks. Even if optical networks can provide high-capacity and low-latency connections between RRHs and BBU pools, optical networks are not always a feasible solution for backhaul networks. In contrast, microwave links and non-line-of-sight wireless links can provide more flexible deployments but introduce latency in the system [117]. Note that the implementation of the wireless network cloud varies. For example, in [118], the central processing and control unit is called virtual base station pool. Nevertheless, we do not have any assumption on the implementation of the wireless network cloud. In the C-RAN considered in this chapter, there are $B$ RRHs with one antenna each, which are denoted as a set $\mathcal{B}$.

Many mobile cloud services require the end-to-end reliable data transfer, TCP, across the two systems. There is a split-TCP proxy at the edge of the wireless network cloud. The split-TCP proxy is the split point for TCP flows. Such split-TCP proxy has been widely used in cloud computing [35] and traditional cellular networks [34]. In the context of cloud computing, split-TCP is also a popular scheme to provide reliable data transfer for cloud services. The client sets up a TCP connection to the nearby split-TCP proxy, then the split-TCP proxy sustains a persistent TCP connection to the data center with a very large TCP connection window [35]. In wireless networks, split-TCP proxy hides the wireless related issues from the wireline host via inserting a split point between the wireless and wired hosts. It locally acknowledges each segment and then stores and forwards the segments on the second TCP connection [34]. The split-TCP proxy can be implemented at system architecture evolution gateway (SAEGW) in LTE systems [119] since the user data flows are tunneled to SAE-GW before being sent to the Internet.

Fig. 4.2 shows the logical relationship of the mobile users, wireless network cloud, and OTT service provider cloud. TCP flows carrying mobile cloud services run from 
the mobile device to the backend server in OTT service provider cloud. Split-TCP proxy residing at the edge of wireless network cloud splits the end-to-end connection between the mobile user and the backend server into two connections and sustains a persistent connection between itself and the backend server. Meanwhile, the wireless network cloud conducts dynamic operations on wireless networks to provide best service for the upper layer. Such dynamic operations include topology configuration and rate allocation. Topology configuration controls how the RRHs cooperate with each other. For instance, in Fig. 4.1, RRHs $B$ and $C$ form a cooperating set to serve the two MCC users together while RRH $A$ itself is another cooperating set. After topology configuration, the wireless network cloud needs to decide the data rates that the MCC users can transmit. Inside a cooperating set, the signals are processed jointly such that there is no interference.

We denote the channel state matrix at time slot $t$ as $S^{t}$, the topology configuration action at time slot $t$ as $\Omega^{t}$. The rate allocation vector has $B$ elements, $\mathbf{R}^{t}=\left[R^{1, t} \ldots R^{B, t}\right]$, where each element is the rate allocation for a user. Here, we assume that there is only one user serviced by each RRH at a time slot, which is commonly assumed in the literature due to the opportunistic scheduling operation in practice [120]. The overall action is $a^{t} \triangleq\left\{\Omega^{t}, \mathbf{R}^{t}\right\} \in \mathcal{A}$, where $\mathcal{A}$ is the set of actions available. In this work, we assume that the power allocated to each user is the same, so that we can focus on the optimal topology configuration and rate allocation problem. On one hand, such power binary power allocation has been shown to be optimal in some scenarios [121]. Thus it is reasonable to decouple power control at the time being. On the other hand, it is straightforward to adapt this framework to support power allocation by taking the possible power allocation scheme into the action space. 


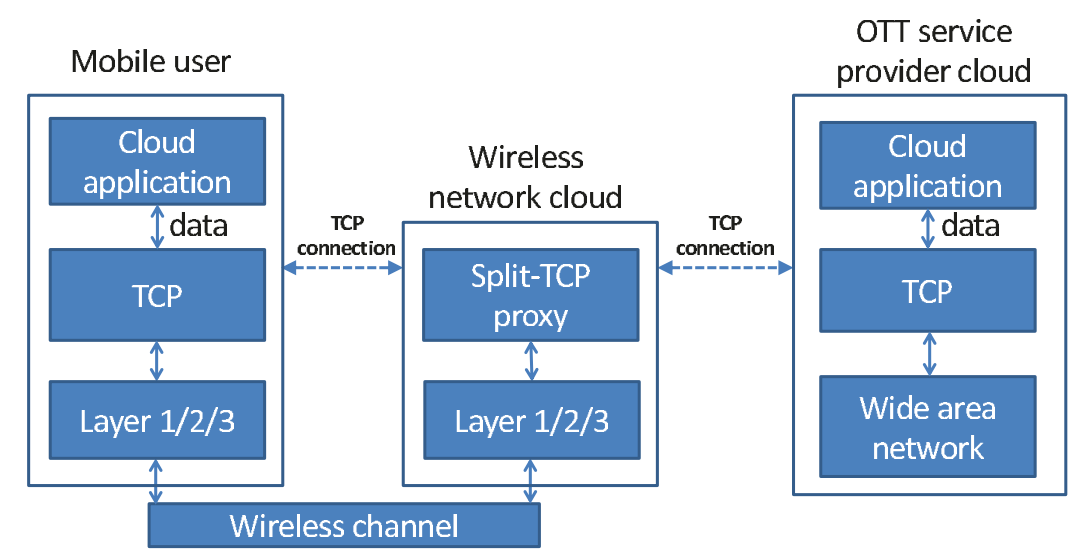

Figure 4.2: Logical protocol stacks of MCC system entities: mobile device, splitTCP proxy, and cloud backend server.

\subsubsection{Physical Layer and Link Layer in C-RAN}

In the following we introduce the physical layer and link layer models. We attempt to make the modeling of these two layers to be as general as possible while sustaining a certain feasibility in performance analysis. This is because essentially C-RAN is supposed to be an open platform to support various technologies at lower layers [110].

Consider a cooperating set $\omega$ whose cardinality is $|\omega|=K$. Signals for mobile users served by RRHs in $\omega$ can be decoded without interfering with each other; while the mobile users served by the non-cooperating RRHs, $\mathcal{B}-\omega$, are interferers to $\omega$. We number the RRHs in $\omega$ from 1 to $K$, and RRHs in $\mathcal{B}-\omega$ from $K+1$ to $B$. Denote the complex channel gain from a mobile device served by RRH $i$ to the antennas of all the RRHs in $\omega$ as $\mathbf{h}_{i} \in \mathbb{C}^{K \times 1}, i=1, \ldots, K, K+1, \ldots, B$. We assume that all mobile devices are allocated the same transmission power $P$, namely, the power allocation matrix of mobile devices in cooperating set $\omega$ is $\sqrt{P} \times \mathbf{I}_{K}$. If the complex data symbols of mobile devices served by cooperating set $\omega$ are $\left[x_{1} \ldots x_{K}\right]$, and the data symbols of mobile devices served by the other RRHs are $\left[\begin{array}{lll}x_{K+1} & \ldots & x_{B}\end{array}\right]$, the received signal of 
the antennas in a cooperating set $\omega$ is given by

$$
\mathbf{y}=\sqrt{P} \sum_{l=1}^{K} \mathbf{h}_{l} x_{l}+\sqrt{P} \sum_{l^{\prime}=K+1}^{B} \mathbf{h}_{l^{\prime}} x_{l^{\prime}}+\mathbf{n},
$$

where $\mathbf{n}$ is a vector of independent complex circularly symmetric additive Gaussian noise with each element $n \sim \mathcal{C N}\left(0, N_{0}\right)$. In the above signal level representation, the first term is the useful signal inside $\omega$, while the second term is interference signal from $\mathcal{B}-\omega$.

As a representative signal processing technique, Minimum Mean Square Error Successive Interference Cancelation (MMSE-SIC) receiver [93, Ch. 10.1] can achieve the multiple access channel capacity. With fixed decoding order, the capacities of users ranging from $1,2, \ldots, K$ are given as follows.

$$
\begin{aligned}
C_{K} & =\log \left(1+\frac{P\left\|\mathbf{h}_{K}\right\|^{2}}{N_{K}}\right), \\
C_{K-1} & =\log \left(1+P \mathbf{h}_{K-1}^{T}\left(N_{K-1} I_{K}+P \mathbf{h}_{K} \mathbf{h}_{K}^{*}\right)^{-1} \mathbf{h}_{K-1}\right), \\
& \cdots \\
C_{1} & =\log \left(1+P \mathbf{h}_{1}^{T}\left(N_{1} I_{K}+\sum_{l=2}^{K} P \mathbf{h}_{l} \mathbf{h}_{l}^{*}\right)^{-1} \mathbf{h}_{1}\right),
\end{aligned}
$$

where $N_{l}, l=1,2, \ldots, K$, are the AWGN noise accounting for the receiver noise $N_{0}$ and the interference from outside $\omega$. Specifically, the total noise at the $l^{\text {th }}$ antenna is $N_{l}=N_{0}+P \sum_{l^{\prime}=K+1}^{B}\left|\mathbf{h}_{l^{\prime}}\right|^{2}$. Note that any other physical layer is likewise applicable to our work.

For a particular user $u$, if the current channel capacity is less than the transmission rate allocated, there is an outage so that the resulting transmission rate is 0 ; otherwise, the resulting transmission rate is equal to the allocated rate. In slot $t$, the performance 
of a user $u$ is partially controlled by the action $a^{t}$ taken by the wireless network cloud. The probability of error without any link-layer retransmission is defined as

$$
p_{1, u}=\operatorname{Pr}\left(r_{u}^{t}\left(a^{t}\right)>C_{u}^{t}\left(a^{t}\right)\right),
$$

where $r_{u}^{t}$ is the rate allocation for user $u$, and $C_{u}^{t}$ is the channel capacity at time slot $t$, which is a random variable since the channel state is unknown. For the link layer, we use hybrid automatic repeat request (HARQ) to reduce unreliability in wireless links. HARQ combines forward error correction and ARQ to increase the communication reliability. We assume a chase combining scheme in the following ${ }^{1}$. The performance of such an HARQ scheme has been analyzed in [122]. It is shown that, for user $u$, the number of packets transmitted, denoted as a random variable $N_{u}$, follows a Gaussian distribution with mean $\mu_{u}$ and variance $\sigma_{u}^{2}$

$$
\begin{aligned}
\mu_{u} & =\frac{1+p_{1, u}-p_{1, u} p_{2, u}}{1-p_{1, u} p_{2, u}}, \\
\sigma_{u}^{2} & =\frac{p_{1, u}\left(1-p_{1, u}+p_{1, u} p_{2, u}\right)}{1-p_{1, u} p_{2, u}},
\end{aligned}
$$

where $p_{1, u}$ is the probability of error after decoding the information block by forward error correction, $p_{2, u}$ is the probability of error after soft combining two successive transmissions of the same information block [122]. Note that $p_{1, u}$ is essentially the outage probability without HARQ defined in (4.3) and that $p_{2, u}$ is usually obtained via link level simulations [122].

Therefore, if the maximum number of transmissions allowed in the link layer is $\nu$,

\footnotetext{
${ }^{1}$ Other HARQ schemes, such as incremental redundancy combining, are applicable in our work as well.
} 
and if the action taken at time slot $t$ is $a^{t}$, the packet error rate is

$$
p_{e, u}\left(a^{t}\right)=\operatorname{Pr}\left(N_{u}>\nu\right)=Q\left(\frac{\nu-\mu_{u}}{\sigma_{u}}\right)
$$

where $Q(\cdot)$ is the well-known $Q$-function. Moreover, the average transmission time of a TCP data packet over wireless links can be computed as

$$
\bar{T}_{\text {wireless }, u}\left(a^{t}\right)=\mu_{u} \frac{L_{\text {data }}+L_{a c k}}{r_{u}},
$$

where $L_{\text {data }}$ and $L_{\text {ack }}$ are the link layer frame size for a TCP data packet and a TCP acknowledgment packet, respectively. We assume the downlink data rate of the C-RAN is the same as the uplink for ease of analysis.

\subsection{C-RAN with Delayed CSI}

In this section, we first introduce the channel modeling based on Finite State Markov Chains (FSMCs), which is essential in taking delayed CSI into account in our formulation. Then we discuss the delayed CSI issue in C-RAN followed by the belief-state concept that captures the uncertainty caused by delayed CSI.

\subsubsection{Finite State Markov Chain Channel Model and De- layed CSI}

We define the vector space consisting of $B^{2}$ elements as the system state $\mathcal{S}$. Assume at time slot $t$, the system state $S^{t}$ is $s \in \mathcal{S}$, it will jump to $s^{\prime}$ at the next time slot. 
With the FSMC channel modeling [90], the state-transition function $A$ is given by

$$
A\left(s, s^{\prime}\right)=\operatorname{Pr}\left(S^{t+1}=s^{\prime} \mid S^{t}=s\right)=\prod_{b=1, u=1}^{b=B, u=B} \operatorname{Pr}\left(I_{b, u}^{t+1} \mid I_{b, u}^{t}\right)
$$

where $I_{b, u}^{t}$ and $I_{b, u}^{t+1}$ are the current state and next state of the FSMC from a transmit antenna of mobile user $u$ to a receive antenna of RRH $b$.

To see how delay comes into C-RAN, we consider a C-RAN shown in Fig. 4.1. The CSI is obtained via the pilot signals received at RRHs. After channel estimation, the CSI will be transmitted over backhaul networks to the wireless network cloud. At the wireless network cloud, a decision about how the RRHs cooperate and the rates at which MCC users can transmit are decided after obtaining CSI. Then, the user data is transmitted. Similar to the measurement and propagation of CSI, user signals are transmitted from MCC users to RRHs, and then are propagated over the backhaul networks. At the moment of decision making, the available CSI is delayed. We can abstract the total delay between the actual channel state at the moment of decision making and the one of observation as one single number. Then, we can map the delay in seconds into the transition steps in Markov chains. Therefore the $d$ steps transition probability is given by multiplying matrix $A$ by $d$ times, $A^{d}$.

\subsubsection{Belief State with Known Delay Steps}

Given the delay in steps, we can derive the belief state, which is the sufficient statistic of the previous action and observation history [74]. A belief state $\mathbf{b}^{t}$ at time slot $t$ is a probability distribution of the state space. Accordingly, the probability that the state at time slot $t$ being $s^{t}$ is given by the corresponding element in $\mathbf{b}^{t}$, denoted as $b\left(s^{t}\right)$. Following [74], we use belief state to express both the vector $\mathbf{b}^{t}$ and its element given a state $b\left(s^{t}\right)$. 
With techniques such as time-stamping, we can know the number of delay steps $d$. With such an assumption, the observation is just the actual state delayed by $d$ steps. Denote the observation at time $t$ as a random variable $O^{t}$. We have $O^{t}=$ $S^{t-d}, t=d+1, \ldots$. Thus we can derive the explicit relation between the current state and observation. The belief state is

$$
\begin{aligned}
b\left(s^{t+1}\right) & =\operatorname{Pr}\left(s^{t+1} \mid o^{t+1}, o^{t}, \ldots\right) \\
& =\operatorname{Pr}\left(s^{t+1} \mid s^{t+1-d}, s^{t-d}, \ldots\right) \\
& =\operatorname{Pr}\left(s^{t+1} \mid s^{t+1-d}\right) \\
& =A^{d}\left(s^{t+1-d}, s^{t+1}\right) .
\end{aligned}
$$

The third line is given by the first-order Markovian property assumed in the FSMC channel model, and $A^{d}$ is the $d$-step probability transition matrix.

\subsubsection{Belief State with Unknown Delay Steps}

If it is difficult to get the number of delay steps, we can still compute the belief state based on Bayesian rule. We first introduce Observation Function B(·) [74]. Assume at time slot $t$, the observation of the system is $o \in \mathcal{O} .{ }^{2}$ The observation function $B(\cdot)$ essentially depicts the probabilistic relationship between an observation $o \in \mathcal{O}$ and a state $s \in \mathcal{S}$. Formally, observation is also a function of the action taken; however, in our problem here observation is independent of the action, so it is defined as

$$
B(s, o)=\operatorname{Pr}(o \mid s)
$$

State-transition function $A(\cdot)$ and observation function $B(\cdot)$ can be obtained via classical algorithms such as Expectation Maximization [102].

\footnotetext{
${ }^{2}$ Obviously, $\mathcal{O}$ is the same as $\mathcal{S}$. We use different notations for clear presentation.
} 
Essentially, provided a new observation at time $t+1, o^{t+1}$, the new belief should reflect the likelihood of ending up in new state $s^{t+1}$, the likelihood of observing $o^{t+1}$, and the pervious belief distribution $\mathbf{b}^{t}$. The rule to update the belief state according the previous belief and current observation based on the Bayesian rule [74] is

$$
\begin{aligned}
& b\left(s^{t+1}\right)=\operatorname{Pr}\left(s^{t+1} \mid o^{t+1}, \mathbf{b}^{t}\right) \\
= & \frac{B\left(s^{t+1}, o^{t+1}\right) \sum_{s^{t} \in \mathcal{S}} A\left(s^{t}, s^{t+1}\right) b\left(s^{t}\right)}{\sum_{s^{t+1} \in \mathcal{S}} B\left(s^{t+1}, o^{t+1}\right) \sum_{s^{t} \in \mathcal{S}} A\left(s^{t}, s^{t+1}\right) b\left(s^{t}\right)} .
\end{aligned}
$$

With the belief state $\mathbf{b}^{t}$, we can compute the probability of error without link-layer retransmission (4.3) as follows.

$$
p_{1, u}=\sum_{c_{u}\left(s^{\prime}, a^{t}\right)<r_{u}^{t}} b\left(s^{\prime}\right)
$$

where $c_{u}\left(s^{\prime}, a^{t}\right)$ is the capacity that user $u$ can achieve given the action $a^{t}$ and the channel state $s^{\prime}$, and $r_{u}^{t}$ is the rate allocation.

\subsection{TCP Throughput over C-RAN in MCC Sys- tems}

In this section, we study the TCP throughput over C-RAN in MCC systems. Then we investigate the user response latency issue. Next, the TCP throughput maximization with response latency constraint problem is formulated as a constrained stochastic optimization problem. Finally, we derive the optimal topology configuration and rate allocation algorithm. 


\subsubsection{Round Trip Time and Split-TCP Throughput}

Split-TCP is a popular reliable data transfer protocol for data center networks [35] and legacy cellular networks [34]. We can expect that it will play an important role in next generation MCC systems. Therefore, in this work, we adopt split-TCP as our transport layer protocol. A widely used TCP throughput model is developed in [123]. It has been used in cross-layer designs to maximize TCP throughput (for instance, [26]). In this section, we extend the existing work to take delayed CSI into account in the TCP throughput model.

We firstly discuss the round trip time (RTT). Fig. 4.3 shows the round trip times for mobile cloud services over C-RAN. There are two types of RTTs [35]. RTT represents the RTT between clients and the split-TCP proxy at the edge of wireless network cloud. $R T T_{2}$ is the RTT between the split-TCP proxy and the backend server in the OTT service provider cloud. We will not discuss the randomness in $R T T_{2}$ because this work is focused on the effect of C-RAN on cloud service.

$R T T_{1}$ consists of $T_{\text {wireless }}$ and $T_{\text {backhaul }}$, which represent the round trip transmission time over the wireless and backhaul networks, respectively. Note that, due to the wireless channel fading, $T_{\text {wireless }}$ is a random variable partly controlled by the action taken by the control unit in the wireless network cloud of C-RAN. The mean value of $R T T_{1}$ is given by

$$
\overline{R T T}_{1}\left(a^{t}\right)=\bar{T}_{\text {wireless }}\left(a^{t}\right)+T_{\text {backhaul }},
$$

where $\bar{T}_{\text {wireless }}\left(a^{t}\right)$ is defined in (4.7).

We assume that the maximum segment size (MSS) is set in such a way that a single segment will fit into a single link-layer frame. That is, there is only one link layer packet for a TCP segment. The queuing delay is not taken into consideration. Since 


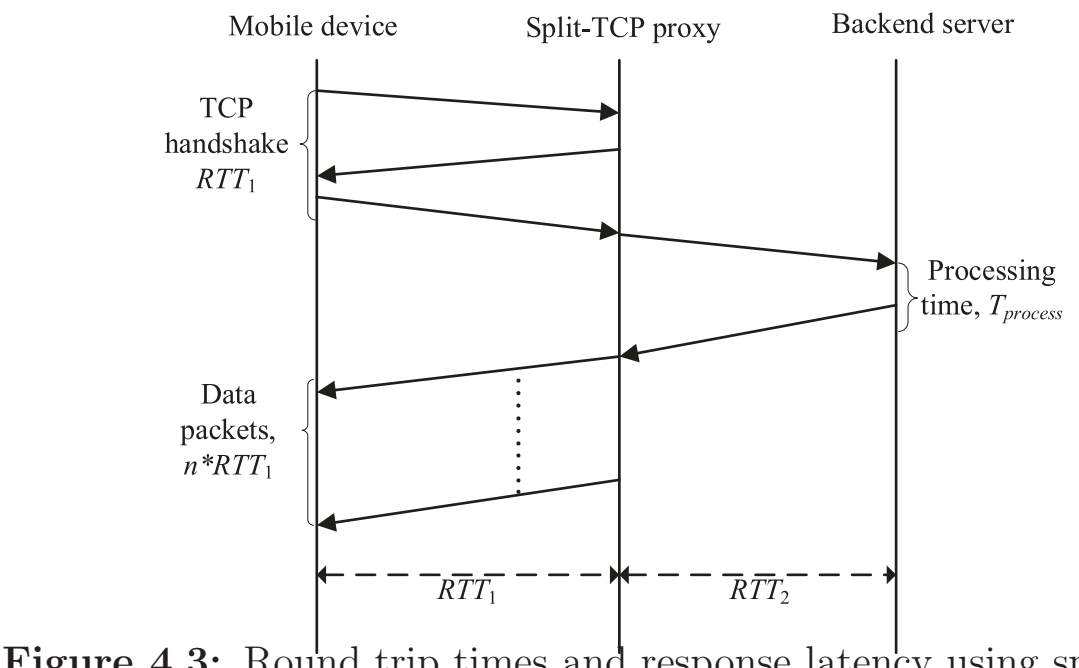

Figure 4.3: Round trip times and response latency using split-TCP.

we focus on the delayed CSI in this work, we can approximately take the queuing delay as a constant contribution to RTT. In terms of implementation, the queuing delay can be approximated by its statistical average. This assumption is reasonable because the time scale of queuing dynamics is generally larger than that of the wireless channel dynamics provided there is a bulk of data to transmit [12]. If the files are small, the queuing delay can be simply ignored. Therefore, we take queuing delay as a constant, which has been considered in $T_{\text {backhaul }}$.

Padhye et al. have developed a model for TCP connections. For user $u$, the average throughput can be derived as [123]

$$
\bar{\eta} \approx \min \left\{\frac{W_{\max }}{\overline{R T T}}, \frac{1}{\overline{R T T} \sqrt{\frac{2 n_{a c k} p_{e}}{3}}+T_{0} \min \left\{1,3 \sqrt{\frac{3 n_{a c k} p_{e}}{8}} p_{e}\left(1+32 p_{e}^{2}\right)\right\}}\right\}
$$

where $W_{\max }$ is the maximum congestion window, $\overline{R T T}$ is the round trip time, $n_{a c k}$ is the number of packets acknowledged by a TCP ACK (generally 2), $T_{0}$ is the initial time-out for the TCP sender, $p_{e}$ is the TCP loss probability. The accuracy of such a model has been verified against real TCP traces in [123]. Note that the throughput of a TCP connection over a radio access network, $\eta_{R A N, u}\left(a^{t}\right)$, is a random variable 
because the actual channel state $S^{t}$ is unknown. $\bar{\eta}_{R A N, u}\left(a^{t}\right)$ is the mean value of it. For a connection in C-RAN, $\overline{R T T}$ and $p_{e}$ are defined in (4.13) and (4.6), respectively.

In split-TCP, the end-to-end throughput is the minimum throughput between the two TCP connections. For a user $u$, denote the average throughput of the TCP connection between the mobile user and split-TCP proxy as $\bar{\eta}_{R A N, u}$, and the one between split-TCP proxy and data centers as $\bar{\eta}_{\text {cloud }}$. The overall average throughput of split-TCP for $u$ given the action taken $a^{t}$ is

$$
\bar{\eta}_{u}\left(a^{t}\right)=\min \left\{\bar{\eta}_{R A N, u}\left(a^{t}\right), \bar{\eta}_{c l o u d}\right\}
$$

\subsubsection{Per-User Response Latency}

Response latency experienced by cloud users is critical for mobile cloud services [35, 115]. Since the main computation tasks are performed in data centers, MCC systems suffer from the response latency caused by processing time and communications among network entities. The processing latency is mainly caused by the hardware and the operating system, which is not the focus of our work. On the other hand, as will be shown in the following sections, the communication latency can be improved by careful design and operation of the C-RAN.

As shown in Fig. 4.3, the connection between the client and split-TCP proxy spends about an $R T T_{1}$ in the hand-shaking phase. $T_{\text {process }}$ is the time needed for the backend severs to process the request. The split-TCP proxy needs to wait an $R T T_{2}$ and $T_{\text {process }}$ in setting up the connection and for the backend server to compute the results and to transmit them to the split-TCP proxy. Using the same assumption as [35], it takes $n * R T T_{1}$ to transmit the results from the split-TCP proxy to the 
MCC users. Denoting the total response latency as $\tau\left(a^{t}, S^{t}\right)$, we have

$$
\tau\left(a^{t}, S^{t}\right)=(n+1) * R T T_{1}\left(a^{t}, S^{t}\right)+R T T_{2}+T_{\text {process }} .
$$

A typical value of $n$ for search engine application is 4 [35]. Recall that $R T T_{1}$ at time slot $t$ is a random variable depending on the actual system state $S^{t}$ and the action taken $a^{t}$. Accordingly the average value of total response latency $\bar{\tau}$ is given by

$$
\bar{\tau}\left(a^{t}\right)=(n+1) * \overline{R T T}_{1}\left(a^{t}\right)+\overline{R T T}_{2}+T_{\text {process }},
$$

where $\overline{R T T}_{1}\left(a^{t}\right)$ is defined in (4.13), $\overline{R T T}_{2}$ and $T_{\text {process }}$ are considered to be constant.

\subsubsection{Maximizing TCP Throughput with Delayed CSI for Mobile Cloud Services}

At time slot $t$, the system state $S^{t}$ is an unobserved random variable. The wireless network cloud selects the cooperating RRHs and allocates the rate for MCC users, denoted as $a^{t}$. Denote the end-to-end throughput of a mobile user $u$ given by (4.15) as a random variable $\eta_{u}\left(a^{t}, S^{t}\right)$, then $\sum_{u=1}^{B} \eta_{u}\left(a^{t}, S^{t}\right)$ is the sum throughput of the system. The number of time slots considered is $h$, which is called the number of horizons in Markov decision process literature [74]. The cumulative rewards over $h$ horizons is $\sum_{t=1}^{t=h} \sum_{u=1}^{u=B} \eta_{u}\left(a^{t}, S^{t}\right)$.

Accordingly, we denote the response latency defined in (4.16) as $\tau_{u}$, and we constrain the latency to be under a threshold $\alpha$. To maximize the sum TCP throughput subject to the response latency constraint, we have the following optimization problem, 


$$
\begin{array}{ll}
\underset{a^{t}, t=1,2, \ldots, h}{\operatorname{maximize}} & \mathbb{E}\left[\frac{1}{h} \sum_{t=1}^{t=h} \sum_{u=1}^{u=B} \eta_{u}\left(a^{t}, S^{t}\right)\right] \\
\text { s.t. } & \mathbb{E}\left[\tau_{u}\left(a^{t}, S^{t}\right)<\alpha\right], u=1, \ldots, B, t=1, \ldots, h .
\end{array}
$$

\subsubsection{Greedy Policy}

The problem in (6.10) is a constrained stochastic optimization problem. We first propose a greedy policy, where the expected objective function value achievable at the current time slot is maximized. In other words, it is optimal for the stochastic optimization problem (6.10) when $h=1$. Then, we will prove that the greedy policy is optimal when we consider multiple horizons. The procedures for the online and offline phases are also illustrated.

Theorem 4.1. The optimal policy for the optimal topology configuration problem is given by (4.19).

$$
\begin{array}{ll}
\underset{a^{t}}{\operatorname{maximize}} & \mathbb{E}\left[\sum_{u=1}^{u=B} \eta_{u}\left(a^{t}, S^{t}\right)\right] \\
\text { s.t. } & \mathbb{E}\left[\tau_{u}\left(a^{t}, S^{t}\right)<\alpha\right], u=1, \ldots, B .
\end{array}
$$

Proof. The optimality of the greed policy is proven via induction. Consider at horizon $h=1$, the optimal action to take is the maximizer of $\mathbb{E}\left[\sum_{u=1}^{u=B} \eta_{u}\left(a^{1}\right)\right]$, which is obviously the action given by greedy policy to maximize the expected rewards in one step.

Assume at horizon $h, h \geq 1$, the optimal policy is the greedy policy given in (4.19). Then at horizon $h+1$, 


$$
\frac{1}{h+1} \mathbb{E}\left[\sum_{t=0}^{t=h+1} \sum_{u=1}^{u=B} \eta_{u}\left(a^{t}, S^{t}\right)\right]=\frac{1}{h+1} \mathbb{E}\left[\sum_{t=0}^{t=h} \sum_{u=1}^{u=B} \eta_{u}\left(a^{t}, S^{t}\right)\right]+\frac{1}{h+1} \mathbb{E}\left[\sum_{u=1}^{u=B} \eta_{u}\left(a^{h+1}, S^{h+1}\right)\right] .
$$

So provided the hypothesis that the greedy policy maximizes the first term in the above equation, the action to take to maximize the total expected rewards is the one to maximize the second item, which is equivalent to the case with horizon 1. Therefore, the greedy policy is the optimal policy for problem (6.10).

From the channel observation and delay, we obtain the belief state, $\mathbf{b}^{t}$, which is the probability mass function of the current CSI. The stochastic optimization problem in (4.19) can be converted into a deterministic optimization problem

$$
\begin{array}{ll}
\underset{a^{t}}{\operatorname{maximize}} & \sum_{u=1}^{u=B} \bar{\eta}_{u}\left(a^{t}\right) \\
\text { s.t. } & \bar{\tau}_{u}\left(a^{t}\right)<\alpha, u=1, \ldots, B .
\end{array}
$$

Techniques to solve such integer programming problems have been well developed. For example, mediate size problems can be solved efficiently by the branch and bound method [124], and very large scale integer programmings can be solved by heuristics such as Genetic algorithm [125].

The algorithm to address the stochastic optimization (6.10) includes an offline and online phases. In the offline phase, for each possible observation, a belief state is computed and the integer programming (4.21) is solved. For the purpose of illustration, we adopt a brute-force approach in the offline phase, as shown in Algorithm 1. The optimal policy computed in the offline phase is utilized in the online phase to make the optimal decisions based on the delayed observations, as shown in Algorithm 
2 .

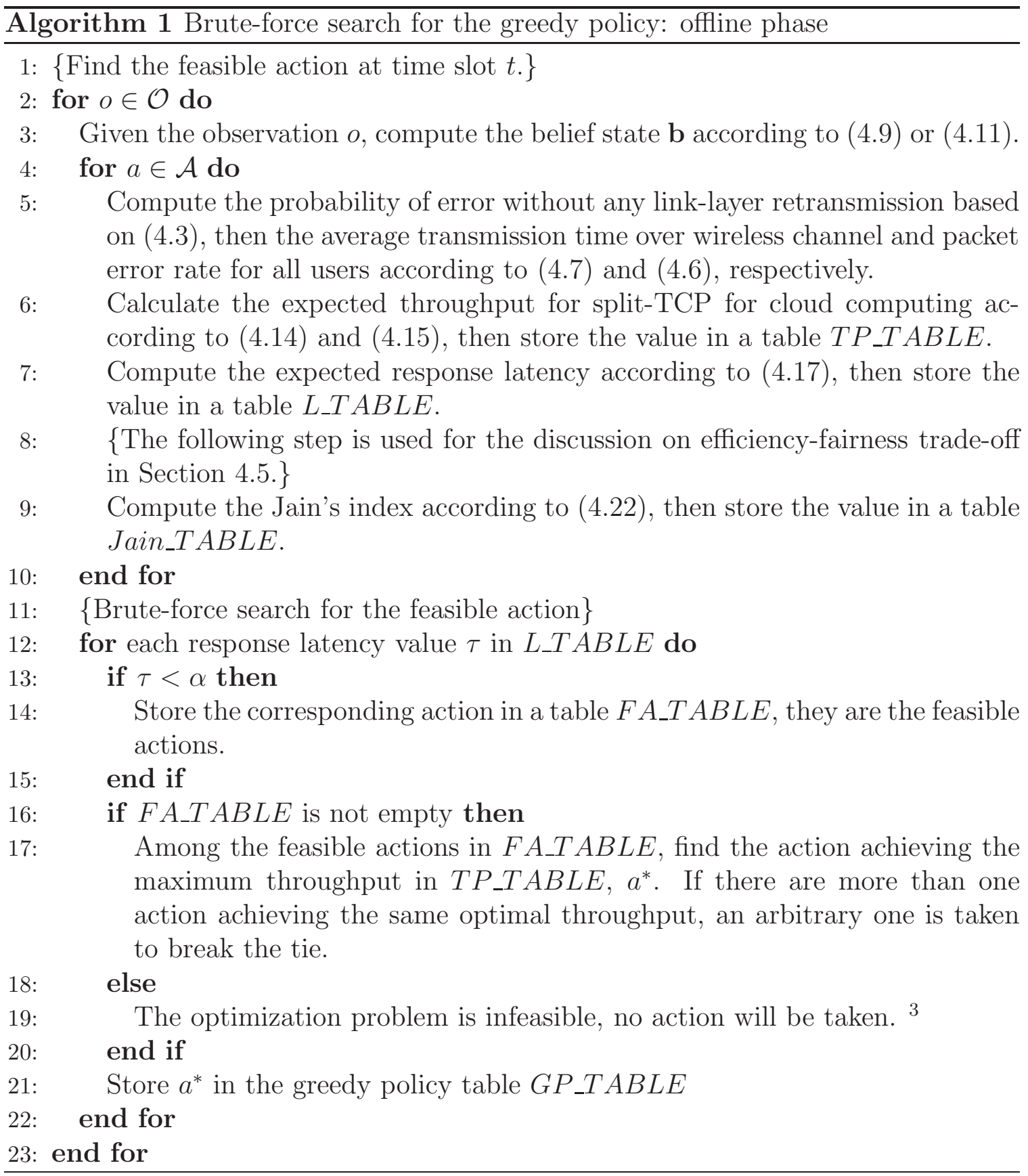

Discussion: In offline phase, the major computation cost lies on storage and manipulations of the station-transition function $A$. In the worst case, $A$ is a $Q^{B^{2}} \times Q^{B^{2}}$ matrix, where $Q$ is the channel quantization level. Fortunately, $A$ is a sparse matrix 

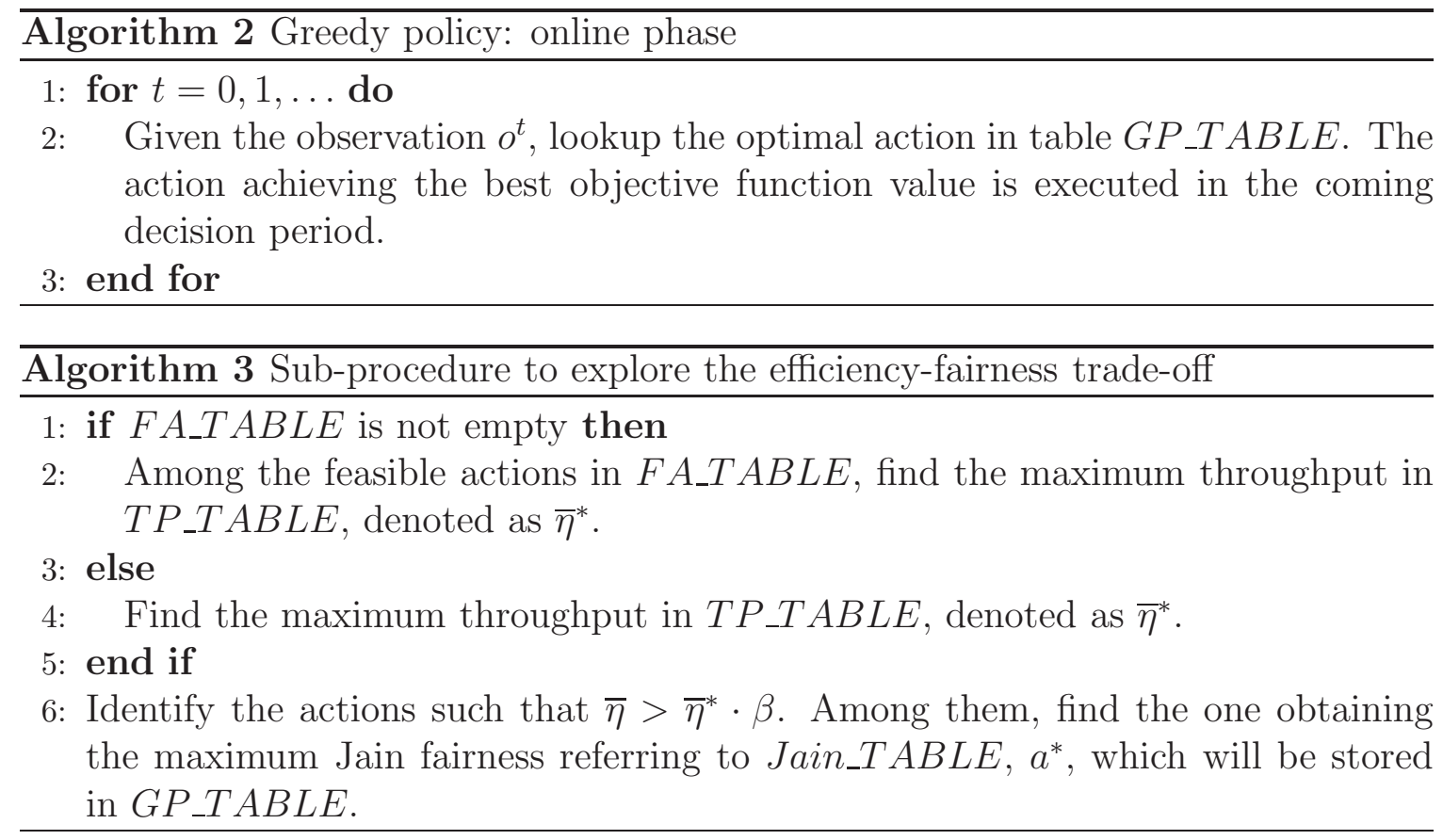

with the majority of the non-zero elements along the diagonal. This hugely reduces the storage requirements and the manipulation complexity [99]. In the online phase, the complexity lies on selecting the best action achieving the best objective function value, which is equivalent to sorting an entry in GP_TABLE. So the complexity is $O(|\mathcal{A}| \log (|\mathcal{A}|))$ since the fundamental limit of run-time complexity for sorting algorithms in worst case is linearithmic [126]. In contrast, the complexity of computing the optimal policy for general partial observable Markov decision processes is PSPACE-complete which is considered to be harder than NP-complete problems [127].

\subsection{Fairness and Efficiency Trade-off}

As in many other multi-user systems, one important performance metric for MCC systems is fairness to the MCC users. This section investigates such metric based on the widely-used Jain's fairness index [128]. For the system we consider, Jain's index 
is defined as

$$
J(\bar{\eta})=\frac{\left[\sum_{u=1}^{u=B} \bar{\eta}_{u}\right]^{2}}{\sum_{u=1}^{u=B} \bar{\eta}_{u}^{2}},
$$

where $\bar{\eta}$ is a vector, consisting of the throughput of all MCC users. Jain's index is between $\frac{1}{B}$ and 1 when the fairness to $B$ users ranging from the least fair to the most fair.

We adopt the definition of optimal efficiency-fairness trade-off in [129]. In particular, we consider the action $a^{*}$ obtaining the optimal efficiency-fairness trade-off, if there is not another action $a \neq a^{*}$ that satisfies either: (a) $\bar{\eta}(a)>\bar{\eta}\left(a^{*}\right)$ meanwhile $J(a) \geq J\left(a^{*}\right)$ or $(\mathrm{b}) \bar{\eta}(a) \geq \bar{\eta}\left(a^{*}\right)$ meanwhile $J(a)>J\left(a^{*}\right)$. In practice, it is possible that even we achieve the optimal trade-off, the fairness index is still not satisfactory. Therefore, in such circumstance, we have to sacrifice some efficiency for the benefit of fairness.

To study the trade-off between the efficiency and fairness, we introduce a parameter $\beta$ in the formulation, which is the percentage of throughput used to enhance the fairness in Jain's index. In particular, denote the maximum sum throughput we can have in (4.21) as $\bar{\eta}^{*}$, we constrain the action such that the sum throughout is not less than $(1-\beta) * \bar{\eta}^{*}$ while maximizing the Jain's index. Namely, we have the following problem,

$$
\begin{array}{ll}
\underset{a^{t}}{\operatorname{maximize}} & J\left(a^{t}\right) \\
\text { s.t. } & \sum_{u=1}^{u=B} \bar{\eta}_{u}\left(a^{t}\right) \geq(1-\beta) * \bar{\eta}^{*} \\
& \bar{\tau}_{u}\left(a^{t}\right)<\alpha, u=1, \ldots, B .
\end{array}
$$

In the above optimization problem, the first constraint will be pushed to meet the 
right hand side as close as possible, since with smaller efficiency, a higher extent of fairness can be expected. To solve this problem, the procedure in Algorithm 1 is modified to explore the trade-off. In particular, steps from 16 to 20 in Algorithm 1 are replaced by the sub-procedure defined in Algorithm 3.

\subsection{Simulation Results and Discussions}

In this section, simulation results are presented to show the effectiveness of the proposed scheme. For the channel model and the physical system model are implemented in matlab. The link layer performance data is fed to network simulator, ns2 (version 2.34) [130]. We conduct simulations using the following settings. There are three RRHs in the C-RAN. The maximum size of a cooperating set is 2 . The wireless channel is Rayleigh fading channel, and the normalized Doppler shift ranges from 0.01 to 0.06 . The bandwidth is $45 \mathrm{KHz}$. The link layer allows frames to be transmitted at most 3 times. For TCP flows, the payload size is 760 bytes. $W_{\max }$ is 6 MSS. Other parameters are shown in Table 4.1. There are two existing schemes used for comparison. In the first one, the effects of imperfect CSI in C-RAN is not considered, and the topology configuration and rate allocation decisions are made based merely on current CSI observations to maximize TCP throughput in MCC systems, which is called Existing scheme - perfect CSI. In the second one, TCP throughput in MCC systems is not considered, and the decisions are made to maximize the physical layer throughput based on imperfect CSI [131], which is called Existing scheme - physical layer throughput. 
Table 4.1: Simulation Parameters

\begin{tabular}{|c||c|}
\hline Parameter & Value \\
\hline Carrier frequence & $2110 \mathrm{Mhz}$ \\
RRH antenna height & $24 \mathrm{~m}$ \\
Mobile device antenna height & $0.5 \mathrm{~m}$ \\
Number of multipath components & 6 \\
Sampling duration & $1 / 500000$ \\
Pathloss & $-174 \mathrm{dBm} / \mathrm{Hz}$ \\
Receiver noise power densisty & $20 \mathrm{dBm}$ \\
Mobile user transmit power & $350 \mathrm{~ms}$ \\
Per user latency constraint & \\
\hline
\end{tabular}

\subsubsection{Performance Improvement}

We measure CSI delay in C-RAN using the unit of samples, the same as in [132]. The performance metrics considered are sum TCP throughput of the MCC users and the average response latency among the MCC users. Fig. 4.4 and Fig. 4.5 show the performance of the three schemes in the low mobility scenario where the normalized Doppler shift is 0.01. Fig. 4.6 and Fig. 4.7 illustrate the results in the high mobility scenario where the normalized Doppler shift is 0.06. In the simulations, the response latency threshold $\alpha$ is set to be 0.35 seconds for the proposed scheme.

From these figures, we can observe that the proposed scheme outperforms the existing ones in terms of both system sum TCP throughput and response latency. In the low mobility scenario, the sum TCP throughput of both the proposed scheme and the existing scheme assuming perfect CSI in C-RAN drops slowly as the CSI delay increases. Nevertheless, the proposed scheme achieves more throughput than the existing scheme, for example, with the delay in CSI being 10 samples, by around $30 \%$. Meanwhile, for the existing scheme assuming perfect CSI, the user response 


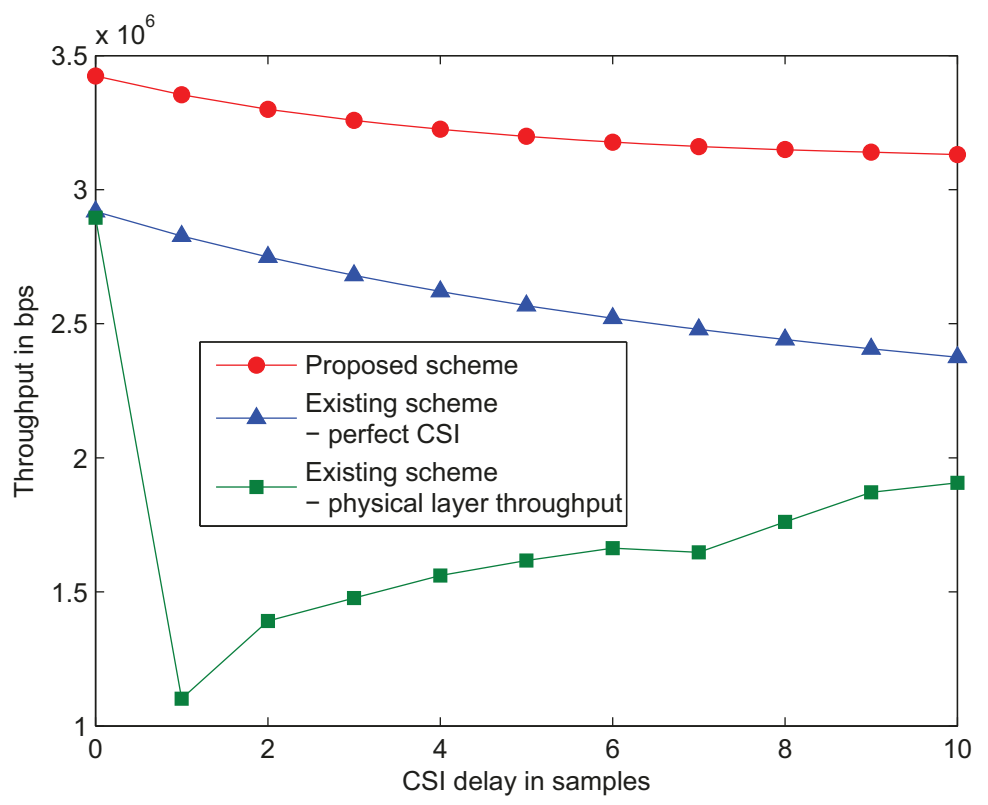

Figure 4.4: The effect of delayed CSI on the end-to-end TCP throughput in the low mobility case with normalized Doppler shift 0.01.

latency increases as the CSI gets more and more delayed. In the high mobility case, the proposed scheme can obtain higher throughput when the response latency is lower than the existing scheme assuming perfect CSI, as shown in Fig. 4.6 and Fig. 4.7. Note that when the delay is zero the proposed scheme still outperforms the existing schemes. That is because in the proposed scheme, we explicitly put the performance of split-TCP connections carrying MCC services into the formulation. In contrast, the existing schemes only aim to maximize physical layer throughput, which would not necessarily bring high performance for MCC.

In terms of throughput, Fig. 4.4 and Fig. 4.6 show that the performance of the existing scheme only considering physical layer throughput is the worst among the three. These two figures indicate that the existing scheme maximizing the physical layer throughput does not guarantee a higher TCP throughput. In terms of response latency, Fig. 4.5 and Fig. 4.7 show the results. In the low mobility case, as CSI delay increases, the response latency of the existing scheme maximizing physical layer 


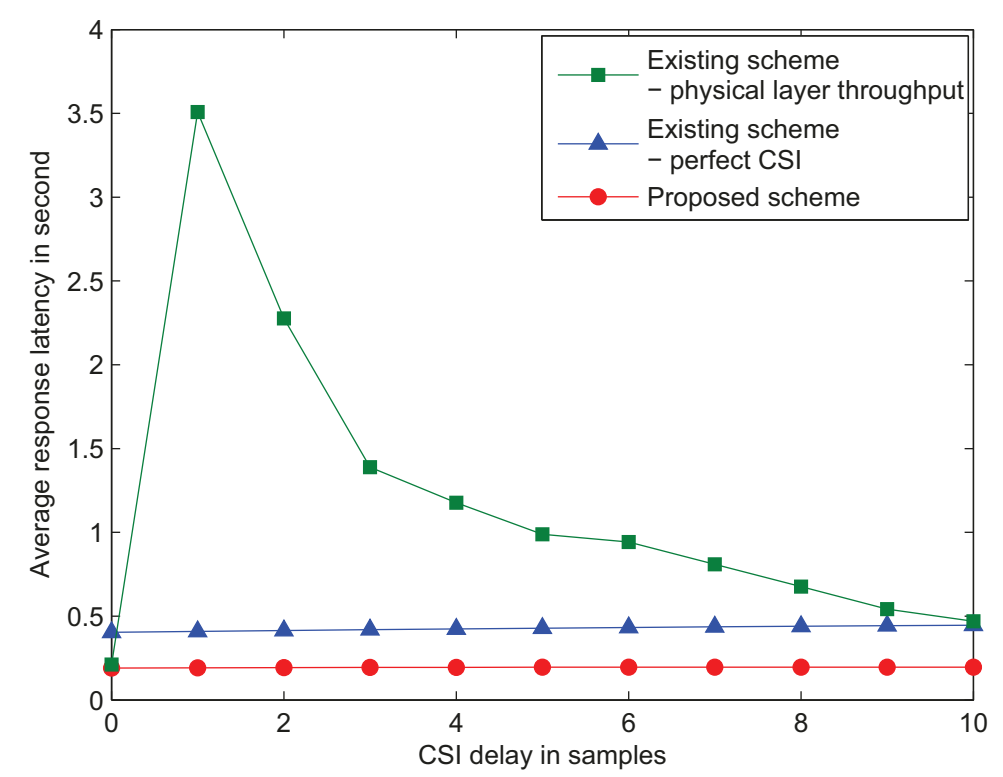

Figure 4.5: The effect of delayed CSI on the average response latency in the low mobility case with normalized Doppler shift 0.01 .

throughput is getting close to that of the existing scheme assuming perfect CSI. In the high mobility case, it outperforms the existing scheme assuming perfect CSI when the CSI delay is larger than 1 samples. As shown in our previous work [131], the existing scheme maximizing the physical layer throughput has better performance than the existing scheme assuming perfect CSI when the criterion is the sum rates of all the MCC users in the system. Furthermore, its advantage decades as CSI delay increases. However, such a scheme is not appropriate when the criterion is the sum TCP throughput of mobile cloud services. The inherent reason is that the behavior of TCP is affected by not only the physical layer throughput but also the round trip time and the end-to-end reliability. The existing scheme maximizing the physical layer throughput only strikes a balance between the outage probability and the rate allocation to achieve maximum physical layer throughout, which might be sub-optimal for MCC systems. So, when the delay is small, e.g., 1 samples, the existing scheme maximizing physical layer throughput has the worst performance. As 


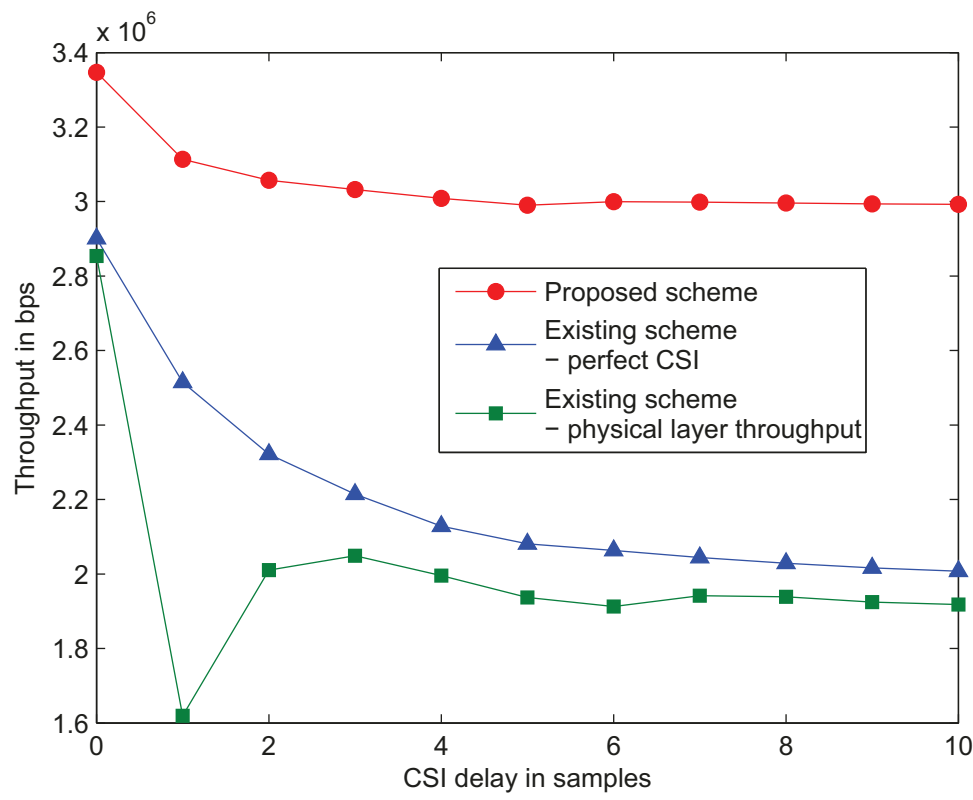

Figure 4.6: The effect of delayed CSI on the end-to-end TCP throughput in the high mobility case with normalized Doppler shift 0.06.

the delay increases, the effectiveness of such a scheme in maximizing physical layer throughput decreases. Consequently, the TCP throughput and latency get close to the one under the existing scheme assuming perfect CSI. That is the reason why we can see a spike in the low CSI delay region in these figures.

Different from these two existing schemes, the proposed one not only considers the issue caused by the delayed CSI, more importantly, it also considers the ultimate performance of split-TCP carrying mobile cloud services. Hence, the simulations results indicate that the proposed scheme is the best one to dynamically configure the C-RAN in MCC systems. Therefore, we believe that it is critical to design and operate the wireless access network in the context of mobile cloud computing, and the joint optimization can have significant advantages compared with the schemes where these two sub-systems are considered separately. 


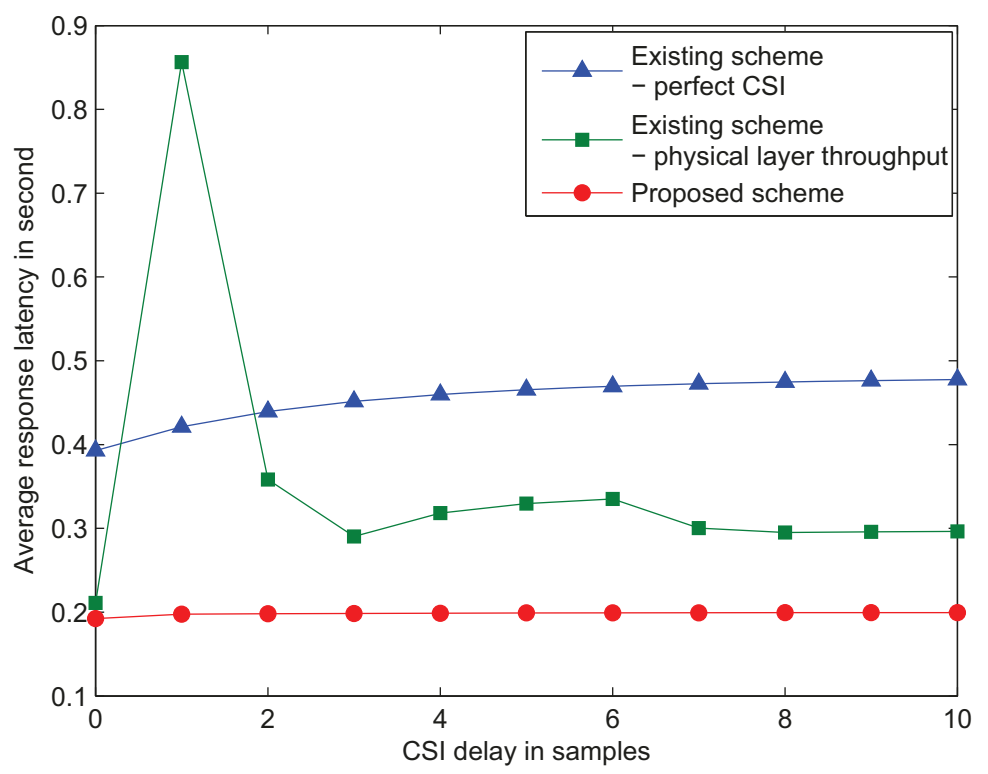

Figure 4.7: The effect of delayed CSI on the average response latency in the high mobility case with normalized Doppler shift 0.06.

\subsubsection{Effects of $R T T_{2}$ and $\bar{\eta}_{\text {cloud }}$}

Fig. 4.8 shows the effect of the RTT over wireline networks, $R T T_{2}$, on the response latency. In the figure, the response latency threshold is $0.35 \mathrm{~s}$. With this threshold, feasible solutions can be found for (6.10), such that the average response latency is bounded under the threshold. Note that if the response latency threshold is very small and/or $R T T_{2}$ is very large, it may not be possible to find feasible solutions to (6.10). In this situation, other mechanisms, such as admission control, should be used to limit the number of MCC users in the system.

For an MCC user, the end-to-end throughput is decided by the two connections separated by the split-TCP proxy. The effect of the throughput of the connection between the split-TCP proxy and the OTT service provider cloud, $\bar{\eta}_{\text {cloud }}$, is shown in Fig. 4.9. With the increase of $\bar{\eta}_{\text {cloud }}$, higher end-to-end throughput is observed. Moreover, the end-to-end throughput hits a plateau when $\bar{\eta}_{\text {cloud }}$ is large enough. That 


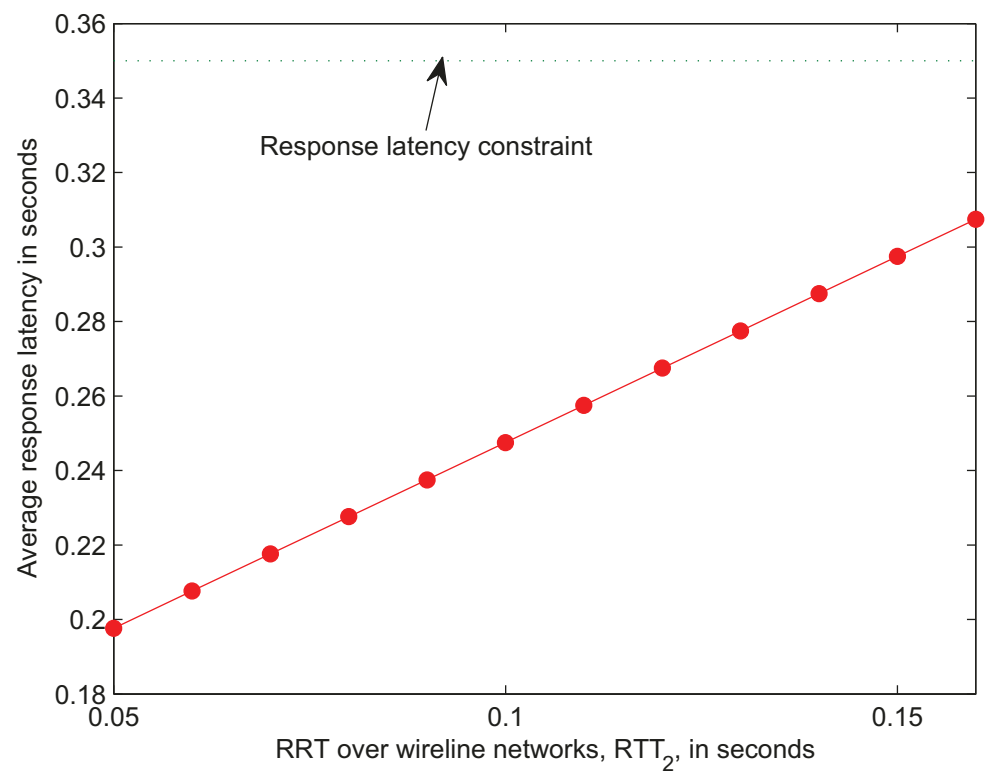

Figure 4.8: The effect of response latency threshold $\alpha$ in the high mobility case with normalized Doppler shift 0.06.

means the bottleneck lies in the radio access network when the throughput between the split-TCP proxy and the OTT service provider cloud is sufficiently large. In this figure, we range the CSI delay from 0 to 8 samples. We can see that with the increase of CSI delay, the end-to-end throughput is getting smaller provided the same $\bar{\eta}_{\text {cloud }}$.

\subsubsection{Efficiency-Fairness Trade-off}

An interesting observation in the simulation is the effect of $\beta$ (the percentage of throughput defined in Section 4.5 to enhance the fairness in Jain's index) with different settings of CSI delay. Fig. 4.10 and Fig. 4.11 show the results for the low mobility and high mobility scenarios, respectively. With the increase of $\beta$, more and more efficiency, i.e., the system throughput, is traded-off for fairness. Yet, interestingly we observe that, with the increase of CSI delay, higher fairness is achieved provided the same setting of $\beta$. This implies that delay in CSI might help increasing the fairness 


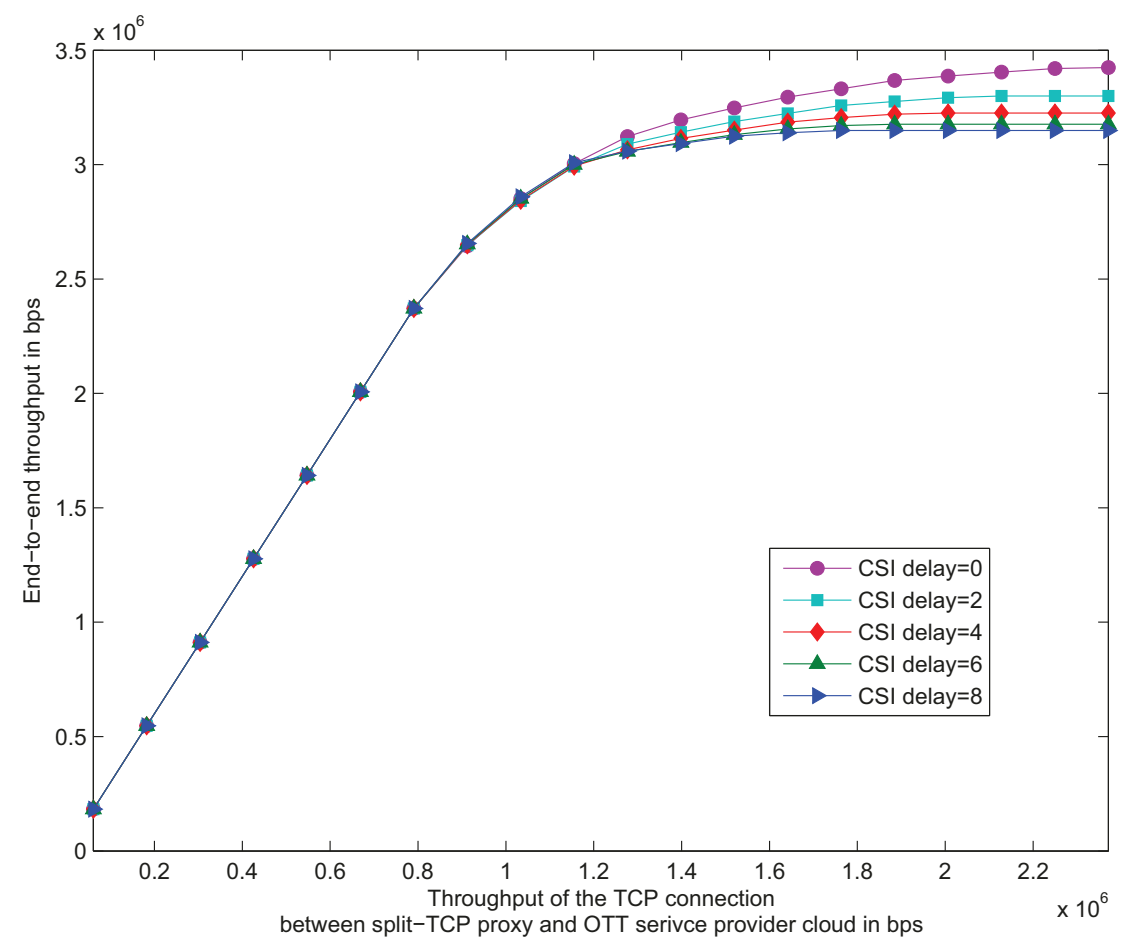

Figure 4.9: The effect of $\bar{\eta}_{\text {cloud }}$ in the low mobility case with normalized Doppler shift 0.01 .

even as we showed before that delayed CSI is bad for system throughput.

\subsection{Chapter Summary}

In this chapter, we jointly studied cloud-RAN and mobile cloud computing in next generation wireless networks. Particularly, the topology configuration and rate allocation problem in C-RAN has been investigated to improve the end-to-end TCP performance of MCC users in next generation wireless networks. We proposed a decision-theoretic approach to tackle the imperfect CSI problem in C-RAN. The response latency experienced by each MCC user was modeled as a constraint. We also studied the trade-off between the efficiency and the fairness among MCC users. Using simulation results, we showed that our proposed scheme can significantly improve the 


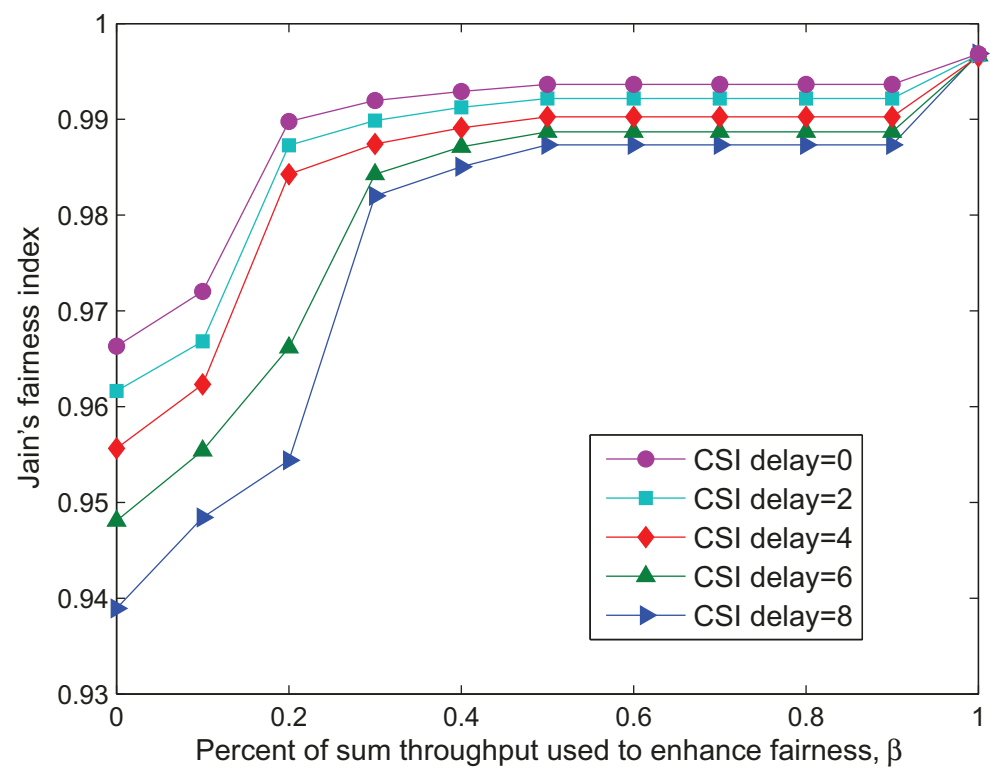

Figure 4.10: Efficiency-fairness trade-off in the low mobility case with normalized Doppler shift 0.01 .

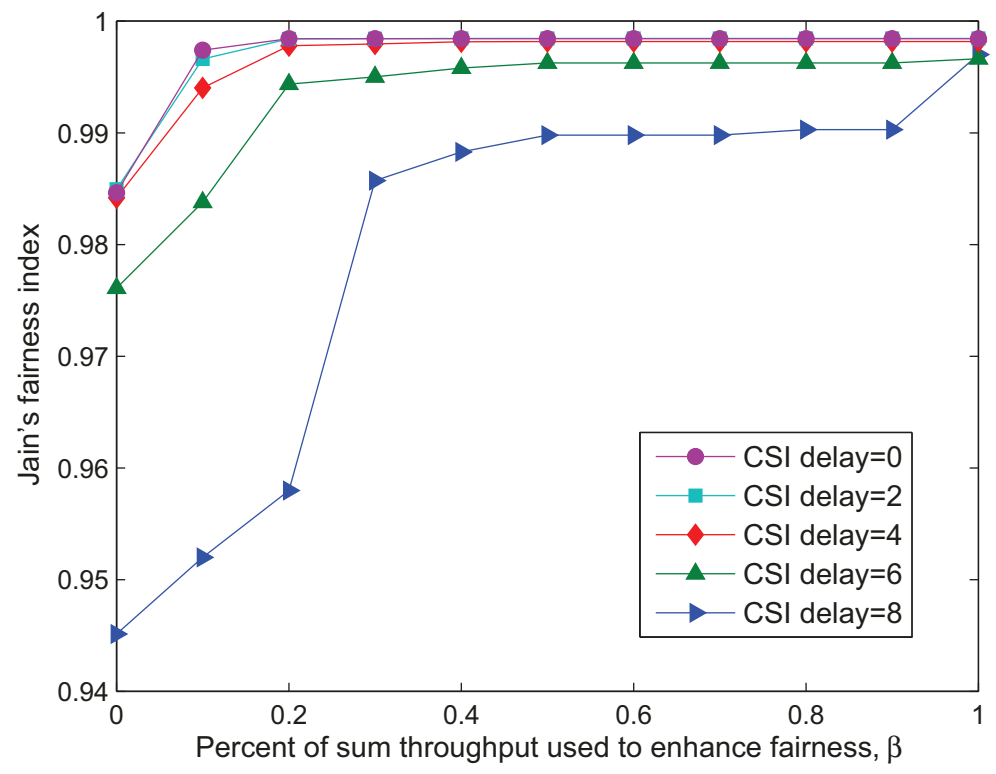

Figure 4.11: Efficiency-fairness trade-off in the high mobility case with normalized Doppler shift 0.06 . 
system performance in terms of throughput and response latency of MCC users. In particular, the delayed CSI in C-RAN has significant effect on the performance, and our proposed scheme is able to reduce such effect, especially in large delay and high mobility scenarios. 


\section{Chapter 5}

\section{Software Defined D2D Communications}

\subsection{Introduction}

SDN and NVF can complement each other in wireless networks [47]. On one hand, for wireless network virtualization, SDN's decoupling principles can enhance performance, simplify compatibility with legacy deployments, and enable operation and maintenance procedures. On the other hand, for SDN controllers, virtual wireless networks provide the infrastructure to run the software on the top.

Although some excellent works have been done on wireless SDN and NVF, recent advances of device-to-device (D2D) communications are largely ignored in this novel framework of SDN and NVF. Recently, the benefits of D2D have been well recognized $[51,52]$. In terms of improving the systematic performance of mobile wireless networks, D2D communications can provide the following gains [55,56]. First, reuse gain allows radio resources to be simultaneously used by both cellular and D2D links. Second, proximity gain empowers high bit rates, low latency and low power consumption thanks to the locations of the two nodes in the D2D link. Third, hop gain enables the source and destination nodes to use a single link rather than both uplink and downlink, which results in two-hop communications. Nevertheless, 
to support D2D, significant amount of modification is needed in both control plane and data plane of radio access networks and core networks. Even it is possible to support D2D by modifying many network entities and protocols in current systems, it will be difficult to provide fast innovation and deployment in the future for new infrastructure-less networking applications [53].

The integration of D2D communications in cellular networks can be greatly facilitated by SDN and NVF. As one of the systematic solutions, applying SDN techniques in virtual wireless networks can provide a versatile framework for the integration of new communications schemes in legacy cellular systems.

In this chapter, we study the integration of D2D communications in the framework of SDN and NVF. Specifically, we consider how to exploit the radio resource pool among multiple Infrastructure Providers (InPs) and D2D communications in virtual wireless networks to maximize the network-wide welfare. The contributions of this chapter are as follows.

- An inherent challenge of software defined D2D communications in virtual wireless networks lies on the fact that the SDN controllers need to have a global view of the network states to optimize the network's performance. Network state information (NSI) consists of channel state information (CSI) and queuing state information (QSI). Due to packet delay and loss, NSI is usually imperfect [133]. Imperfect NSI has significant impact on the performance of not only D2D networks, but also wireless networks in general. Indeed, the capacity of channels with incomplete NSI is largely unknown in wireless networks [85]. To the best of our knowledge, this work is the first one on addressing the imperfectness of NSI for software defined D2D communications in virtual wireless networks.

- User utility is modeled as a function of the resource sharing decision. For a particular unit of radio resource, different resource sharing decisions result in 
different levels of satisfaction due to the channel dynamics, the randomness of the traffic and various prices via either D2D or a particular InP. We further formulate the resource sharing problem with imperfect NSI as a discrete stochastic optimization problem, in which the sum of long term user utility is maximized.

- We develop discrete stochastic approximation (DSA) algorithms to solve the discrete stochastic optimization problem. We first develop aggressive discrete stochastic approximation for static channels, then introduce adaptive step size DSA for time-varying channels.

- Extensive simulations show that wireless mobile users can benefit from both wireless network virtualization and software defined D2D communications, and our proposed scheme can achieve considerable performance gain under practical network settings.

The rest of the chapter is organized as follows. Sect. 5.2 shows the system model of the software defined D2D communications in virtual wireless networks. Sect. 5.3 and Sect. 5.4 discuss the resource sharing problem under perfect and imperfect network state information, respectively. Sect. 5.5 discusses the simulation results. Finally, we conclude this study with future work in Sect. 5.6.

\subsection{System Description}

In this section, we shall define the software defined virtual wireless network framework, the software defined approach to support D2D communications, and the resource sharing scenarios. 


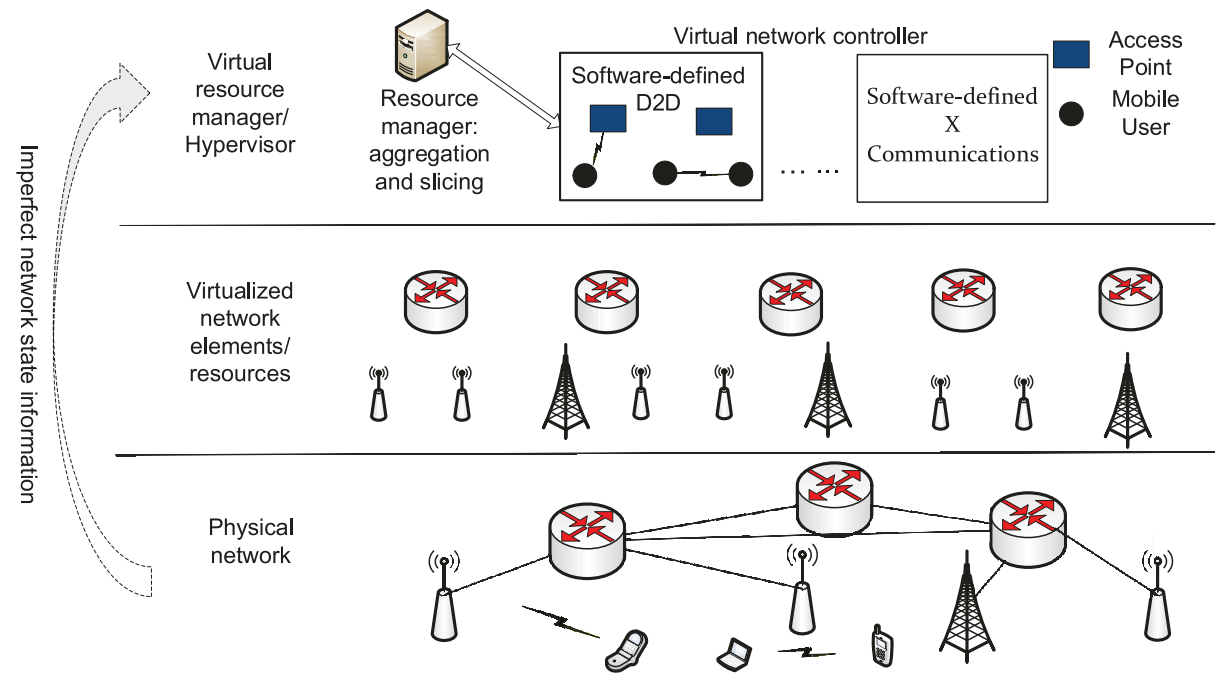

Figure 5.1: Virtual wireless network.

\subsubsection{A Software Defined Framework Utilizing Virtual Wire- less Networks}

The management of the virtual networks is extremely complicated. One solution to handle these complications is to decouple the control and data planes and to have the control logics located inside a central controller via the software defined networking paradigm. Fig. 5.1 shows a possible system framework for this purpose. The physical networks owned by multiple InPs are first mapped into virtual infrastructures and elements. Then the virtual entities are aggregated and sliced into different virtual networks by the virtual resource manager or the hypervisor [48]. With the architecture shown in Fig. 5.1, we can deploy flexible network controllers under arbitrary combinations of wireless technologies such as $2 \mathrm{G} / 3 \mathrm{G} / 4 \mathrm{G} / \mathrm{WiFi}$. The network controllers are on the top of the system. They define the supported communication modes. Since they are all software independent of the underlying hardware, the introduction of new communication schemes or policies is very flexible.

The network controller concept is similar to the network operator concept in [134]. However, the single network operator in [134] is only responsible for spectrum access 
control, and its main role is to provide an abstract underlying channel conditions for multiple SPs. In Fig. 5.1, network controllers can have more functionalities, including topology control of the whole network. In terms of supporting the integration of the infrastructure-less networking schemes into legacy cellular networks, we can have software defined D2D communications. Moreover, in the future, such framework is flexible to support more communication schemes, for example, multi-hop wireless communications.

The controllers manage the network based on the NSI provided by the lower tier in Fig. 5.1. One of the challenges in such systems is that it requires NSI to make resource sharing decisions, however, the observation of the network state is inaccurate in general due to the limitation in measurement and the dynamics of the whole system. Take CSI as an example, the channel estimation and measurement can only represent the actual channel state within limited granularity even in a point-topoint wireless communication system. The imperfectness is even worse in the virtual wireless networks since the NSI is transmitted to the central controller via rate-limited backhaul networks, which introduce transmission delays.

\subsubsection{SDN Approach to Support D2D Communications}

Traditionally, in a simplified cellular network with D2D communications enabled, a gateway is responsible for detecting the user traffic so that the potential D2D users can be paired if it is feasible. Under the legacy wireless mobile network framework, to support D2D communications requires introducing new functions (e.g., traffic earmarking for gateways, new radio resource management schemes for access points) and new signalling protocols between network entities. An example of the session setup and management approaches in a LTE-Advanced context can be found in [53]. In the paper, the solution to address the limitation in terms of upgrading many elements is 
to have software defined network architecture in a virtual environment, such as the one illustrated in Fig. 5.1.

In particular, for D2D communications, the procedure of peer discovery and radio resource management can be done jointly by the network controller. In the peer discovery phase, the network controller accesses the routing table (if a open-flow type of implementation is adopted, it is the flow table), and decides if the session can be potential D2D communication pair. If the source and the destination nodes are recognized as potential D2D pair, the network controller further decides whether to perform D2D transmission or to utilize a access point to relay the information. Moreover, since it is a virtual wireless network, it will also consider which access point is responsible for relaying the traffic if the access point relay mode is selected.

\subsubsection{Resource Sharing in a Virtual Wireless Network with D2D}

In this chapter, we only consider the interactions and optimizations between InPs and MVNOs in the virtual wireless network. Without loss of generosity, we assume that there is a single MVNO in the virtual network and that the MVNO manages the radio spectrum resource pool. The key question in such setting is how to make the best use of the precious radio resources. Fig. 5.2 demonstrates an instance of the resource sharing problem. In the case study, there is a unit of radio resource, which can be used either in D2D modes or in relay mode (via different InP's infrastructure). Here we have the degrees of freedom from the networks ( $\operatorname{InP} i$ or $\operatorname{InP} j$ ) and the user pairs (Pair $A$ or Pair $B$ ) in utilizing the unit of radio resource. In particular, Fig. 5.2(b) and (c) show the diversity from network selection and Fig. 5.2(a) and (d) illustrate the diversity from use pair selection.

Assume that the InPs in the virtual wireless network are $\mathcal{I}=\{1, \ldots, i, \ldots I\}$ with 


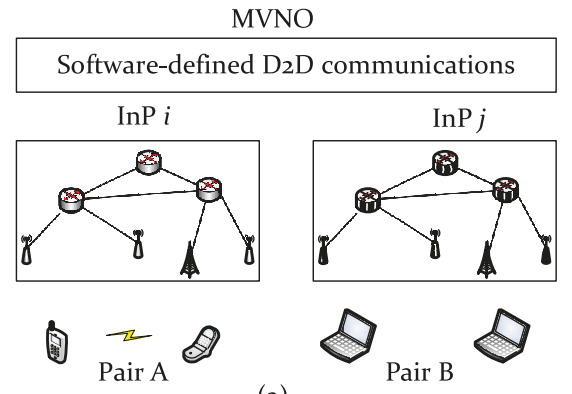

(a)

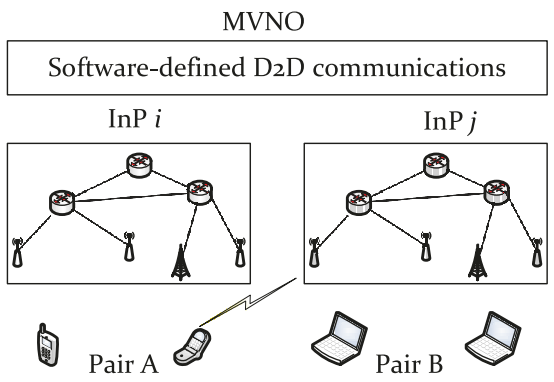

(c)

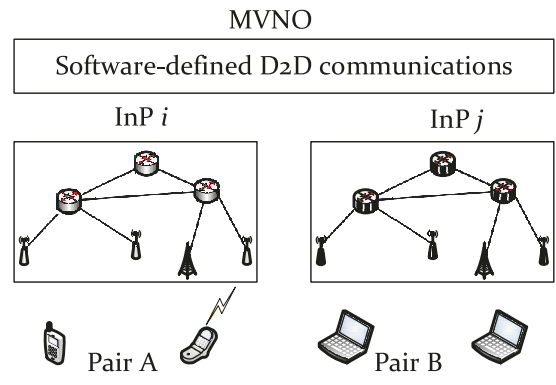

(b)

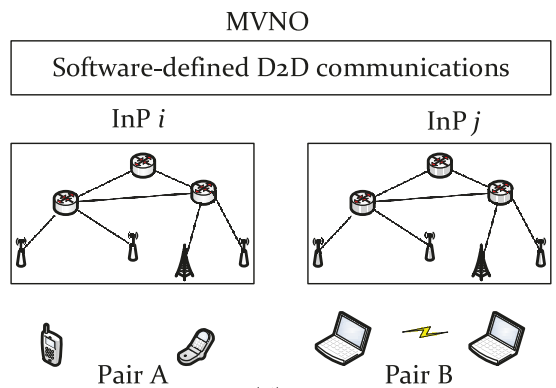

(d)

Figure 5.2: Case studies of resource sharing in virtual wireless networks. (a) The radio resource is used in $\mathrm{D} 2 \mathrm{D}$ communications for Pair $A$; (b) Pair $A$ 's uplink to InP $i$ 's network; (c) Pair $A$ 's uplink to InP j's network; (d) D2D for Pair $B$.

different prices $\mathbf{C}=\left[c_{1}, \ldots, c_{i}, \ldots, c_{I}\right]$ for each of them. That means to utilize $\operatorname{InP} i$, the cost is $c_{i}$ dollars ${ }^{1}$. If the radio resource is to be used in a D2D way, the cost is denoted as $c_{0}$. Note that the radio resource is in the control of MVNO, so $\mathbf{C}$ is independent of radio resource management mechanisms. For an $\operatorname{InP} i$, it has a set of assess points (APs), denoted as $\mathcal{A}_{i}$. Then the set of all APs is $\mathcal{A}=\bigcup_{i \in \mathcal{I}} \mathcal{A}_{i}$.

Since there usually are more resources allocated in downlink than in uplink, if the traffic turns out to be symmetrical, the uplink is the bottleneck from the point of view of end to end communications. Thus this work focuses on the uplink when the relay mode is selected. Denote the set of source nodes as $\mathcal{S}$. The total number of wireless links concerned is $|\mathcal{S}| \times|\mathcal{A}|+|\mathcal{S}|$, in which there are $|\mathcal{S}| \times|\mathcal{A}|$ links in uplink and $|\mathcal{S}|$ links in D2D. Assume the radio resource pool is $\mathcal{N}$ with $|\mathcal{N}|$ units of radio resource

\footnotetext{
${ }^{1}$ We can further suppose that, even in the same $\mathrm{InP}$, the price to access different access points is different according to geographic locations and time.
} 
at MVNO. So the channel of the $|\mathcal{N}|$ units of radio resource at decision epoch $t \mathbf{H}^{t}$ is a vector of $(|\mathcal{S}| \times|\mathcal{A}|+|\mathcal{S}|) \times|\mathcal{N}|$ dimensions.

\subsection{Resource Sharing in Virtual Wireless Net- works with Perfect Network State Information $(\mathrm{NSI})$}

The resource sharing problem is how to allocate the resources to the flows in virtual wireless networks. Provided perfect NSI related to the $|\mathcal{N}|$ units of virtual radio resources, the optimization problem is to search an optimal decision in a space of $\left(\begin{array}{c}|\mathcal{S}| \times|\mathcal{A}|+|\mathcal{S}| \\ N\end{array}\right)$ options. Denote the set of all possible combination as $\Omega$, and the decision variable at each decision epoch $t x^{t} \in \Omega$. When the traffic is non fullbuffer, the queue dynamics need to be considered in resource sharing. Without loss of generality, we assume there is one flow between each wireless mobile user pair. The queue lengths at the destination nodes are not relevant to the resource sharing problem since the data packets are delivered to the upper layer whenever they arrive the destination nodes. So the queue state information at epoch $t$ is a $|\mathcal{S}| \times 1$ vector denoted as $\mathbf{Q}^{t}$. Denote the arrivals between two decision epochs $t$ and $t+1$ as $\boldsymbol{\lambda}^{t}$, which is also a $|\mathcal{S}| \times 1$ vector. For a flow $f$ originating from node $s$, the arrival between two epochs is denoted as $\lambda_{s}^{t}$. Fig. 5.3 illustrates the relationship of the decision epochs and the random variables.

Denote the length of a queue at time $t$ in node $s \in \mathcal{S}$ as $Q_{s}^{t}$, and the channel gain via radio resource $n \in \mathcal{N}$ from node $s$ to access point $a \in \mathcal{A}$ or to destination node $d$ as $h_{s, a}^{t, n}$ or $h_{s, d}^{t, n}$. The queueing dynamics of the source node is described as, 


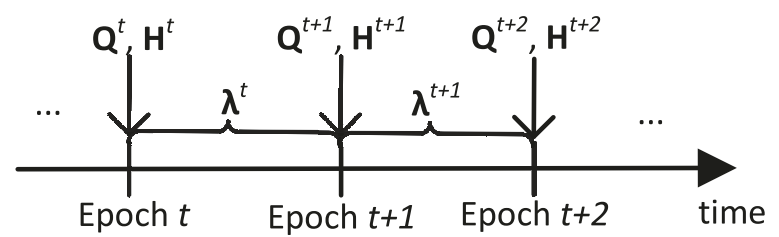

Figure 5.3: Decision epochs, random traffic arrivals, and queuing dynamics in a time sequence schema.

$$
Q_{s}^{t+1}=Q_{s}^{t}-\sum_{a \in \mathcal{A}} \sum_{n \in \mathcal{N}} \tilde{\mu}_{s, a}^{t, n}\left(x^{t}, Q_{s}^{t}, h_{s, a}^{t, n}\right)-\sum_{n \in \mathcal{N}} \tilde{\mu}_{s, d}^{t, n}\left(x^{t}, Q_{s}^{t}, h_{s, d}^{t, n}\right)+\lambda_{s}^{t},
$$

in which $\tilde{\mu}_{s, a}^{t, n}(\cdot)$ is the actual service rate of the flow from source node $s$ to AP $a \in \mathcal{A}$, and $\tilde{\mu}_{s, d}^{t, n}(\cdot)$ from $s$ to $d$. $\lambda_{s}$ is the traffic arrival rate at source node $s^{2} \cdot \tilde{\mu}_{s, a}^{t, n}(\cdot)$ is computed as follows,

$$
\tilde{\mu}_{s, a}^{t, n}\left(x^{t}, Q_{s}^{t}, h_{s, a}^{t, n}\right)= \begin{cases}\min \left\{\mu_{s, a}^{t, n}\left(h_{s, a}^{t, n}\right), Q_{s}^{t}\right\}, & \text { if } x=s \rightarrow a \text { using resource } n \\ 0, & \text { otherwise }\end{cases}
$$

where $\mu_{s, a}^{t, n}\left(h_{s, a}^{t, n}\right)$ is the link capacity decided by the channel realization $h_{s, a}^{t, n}$, and $s \rightarrow a$ means the selected transmission mode is from node $s$ to AP $a$. Likewise, the actual service rate via D2D is given as

$$
\tilde{\mu}_{s, d}^{t, n}\left(x^{t}, Q_{s}^{t}, h_{s, d}^{t, n}\right)= \begin{cases}\min \left\{\mu_{s, d}^{t, n}\left(h_{s, d}^{t, n}\right), Q_{s}^{t}\right\}, & \text { if } x=s \rightarrow d \text { using resource } n \\ 0, & \text { otherwise }\end{cases}
$$

At each decision epoch, the software defined D2D controller obtains a noisy

\footnotetext{
${ }^{2}$ Admission control can be considered by controlling $\lambda_{s}^{t}$.
} 
observation of the NSI (merely CSI is needed for full-buffer traffic case), then decides whether the flows are transmitted via direct wireless links between the sourcedestination pairs or via the access point. The length of the period between two consecutive decision epochs is a tunable system parameter. In principle, the finer the granularity of the period, the better performance can be achieved with the higher overhead in control signaling.

\subsubsection{Utility Function}

The wireless users' behavior is heavily influenced by the pricing mechanism of MVNOs and InPs. Thus, we consider the prices offered by the virtual wireless network in the utility function. The immediate user utility is defined as a function of the channel and queue states and the decision variable, $f\left(\mathbf{Q}^{t}, \mathbf{H}^{t}, x^{t}\right)$. Denote the flows in the network as a set $\mathcal{F}$. If a unit of radio resource is to be used by a flow $f \in \mathcal{F}$ via a specific communication mode (either via an $\mathrm{InP}$ or via $\mathrm{D} 2 \mathrm{D}$ ), the utility achieved is decided by the end-to-end data rate $R_{f}$ and the cost of the selected communication mode $c_{f} \in\left\{c_{0}, c_{1}, \ldots, c_{I}\right\}$. ${ }^{3}$ Since we assume that when the relay mode is selected, the bottleneck link is in the uplink, we have the following,

$$
R_{f}\left(Q_{s}^{t}, \mathbf{H}^{t}, x^{t}\right)=\sum_{a \in \mathcal{A}} \sum_{n \in \mathcal{N}} \tilde{\mu}_{s, a}^{t, n}\left(x^{t}, Q_{s}^{t}, h_{s, a}^{t, n}\right)+\sum_{n \in \mathcal{N}} \tilde{\mu}_{s, d}^{t, n}\left(x^{t}, Q_{s}^{t}, h_{s, d}^{t, n}\right)
$$

Furthermore, the network-wide utility is defined as the ratio between the actual data rate and the price. With all the flows in $\mathcal{F}$, we have

$$
f\left(\mathbf{Q}^{t}, \mathbf{H}^{t}, x^{t}\right)=\sum_{f \in \mathcal{F}} \frac{\sum_{a \in \mathcal{A}} \sum_{n \in \mathcal{N}} \tilde{\mu}_{s, a}^{t, n}\left(x^{t}, Q_{s}^{t}, h_{s, a}^{t, n}\right)+\sum_{n \in \mathcal{N}} \tilde{\mu}_{s, d}^{t, n}\left(x^{t}, Q_{s}^{t}, h_{s, d}^{t, n}\right)}{c_{f}\left(x^{t}\right)} .
$$

\footnotetext{
${ }^{3}$ Rigidly, $\left\{c_{0}, c_{1}, \ldots, c_{I}\right\}$ is a tuple instead of a set since the price offered by different InPs can be the same.
} 
With imperfect NSI, $f\left(\mathbf{Q}^{t}, \mathbf{H}^{t}, x^{t}\right)$ is a random variable.

Essentially, the resource sharing problem is to select the optimal transmission mode and resource utilization approach from $\Omega$. If we have perfect knowledge of the channel and the queue states, we can have a formulation of the resource sharing problem via deterministic optimization. Such optimization can be solved by ranking all the possible values of the objective function (5.5), and then choosing the $x$ with the biggest objective function value. Other system design aspects, such as power control, can also be considered jointly. Yet, in fact, it has been well recognized that most of the performance gain achieved by water-filling power control in maximizing the ergodic sum-rate [135] is from multi-user diversity [136]. Thus the joint power control might not bring much gain.

\subsection{Resource Sharing with Imperfect Network S- tate Information (NSI)}

As we can see from the previous section, NSI is needed to make resource sharing decisions. However, the observation of the network state is inaccurate in general due to the limitation in measurement and the dynamics of the whole system. The imperfectness is even worse in the virtual wireless networks since the NSI is transmitted to the central controller via rate-limited backhaul networks, which introduce transmission delays. Therefore, in this section, we first formulate the resource sharing problem with full-buffer traffic and imperfect CSI, which does not need to take QSI into account. Then we take the random traffic arrival into consideration where only noisy CSI and QSI can be obtained. 


\subsubsection{Full Buffer Traffic and Imperfect CSI}

The assumption of the availability of CSI is the same as $[59,60]$, where the inaccuracy in CSI is modeled as additive noise. The noisy version of the channel state $\hat{\mathbf{H}}$ is given by

$$
\hat{\mathbf{H}}=\mathbf{H}+\mathbf{E},
$$

in which the noise term $\mathbf{E}$ is usually assumed as i.i.d Gaussian [137]. For each decision epoch, the network-wide utility defined in (5.5) is a random variable since $\mathbf{H}^{t}=$ $\hat{\mathbf{H}}^{t}-\mathbf{E}^{t}$ is a random variable and $\mathbf{Q}^{t}$ is ignored in this subsection. With imperfect CSI, we can only optimize the expectation of the sum utility,

$$
\underset{x \in \Omega}{\operatorname{maximize}} \mathbb{E}\left[f\left(\mathbf{H}^{t}, x^{t}\right)\right]
$$

A solution for the above discrete stochastic optimization is to exhaustively search the feasible set $\Omega$ [138]. To do so, we first need to compute the empirical average of the objective function assuming a particular action $x$ is taken. Over a very large period of time $T$, according to strong law of large numbers, we have

$$
\hat{f}(x) \triangleq \frac{1}{T} \sum_{t=1}^{T} f\left(\mathbf{H}^{t}, x\right) \rightarrow \mathbb{E}\left[f\left(\mathbf{H}^{t}, x\right)\right]
$$

Then the optimal action is

$$
x^{*}=\underset{x \in \Omega}{\operatorname{argmax}} \hat{f}(x) .
$$


The exhaustive search approach has two disadvantages. First, it requires a extremely large amount of computation. To be exact, the number of times computing

the objective function value is $T \cdot\left(\begin{array}{c}|\mathcal{S}| \times|\mathcal{A}|+|\mathcal{S}| \\ N\end{array}\right)$. Even it might be possible to do so for a practical virtual network, the efficiency of the computation is very low because ideally only the empirical average of the optimal objective function is needed. Second, the actual virtual wireless network is dynamic system and the channel states are time-varying. But to have statistically meaningful empirical average, we need to have a sufficiently large $T$, which is not achievable if the channels are changing in a time period less than $T$.

Discrete stochastic approximations $[75,79]$ can address these drawbacks. Stochastic approximation algorithms are a type of iterative optimization algorithms to find the optimal solution of the objective functions, which cannot be computed explicitly but there are estimations of them [139]. Discrete stochastic approximations are a subset of general stochastic approximations where the decision variables are discrete ones. These algorithms have been employed in [79] for MIMO antenna selection and in [140] for CDMA code optimization.

\section{Discrete Stochastic Approximation Algorithm for Static Channel}

The DSA algorithm to solve the resource sharing problem (5.7) is illustrated in Algorithm 4 . The basic spirit of the algorithm is to randomly explore $\Omega$ while keeping track of the number of visited resource sharing mode. In each round of iteration, the algorithm tends to pick up the resource sharing mode with higher network-wide user utility. We use a $|\Omega| \times 1$ vector $\pi^{t}$ to record the relative frequency of each mode that has been visited up to time $t$, i.e.,

$$
\pi^{t}\left[x^{t}\right]=\frac{\# \text { of visits to } x^{t}}{t}
$$


In the initialization phase, the elements of $\pi^{0}$ are set to 0 except for the one corresponding to the initial selected mode.

Each iteration in Algorithm 4 consists of 4 steps. In Step 1, a resource sharing mode $\tilde{x}^{t}$ is uniformly chosen, and the corresponding objective function value is evaluated. The same as in [79], we assume that the estimation of the network state is unbiased. We can achieve unbiased estimates by using different training preambles in channel estimation. In Step 2, if the randomly chosen objective function value is larger than that of the current state $x^{t}$, it is accepted as the next visited state. In Step 3 the state occupation probabilities are updated. $e_{x}$ is a $|\Omega| \times 1$ vector, in which all the elements are 0 except for the $x^{\text {th }}$ one with a value of 1 . Note that the step size in each iteration is $\frac{1}{t}$, that means as time goes, the algorithm is getting more and more conservative to explore new resource sharing possibilities. That is the reason why the algorithm is also called aggressive discrete stochastic approximation [140]. In step 4, if the frequency of the newly visited state is higher than the resource sharing mode selected in last iteration, it is chosen to be the new resource sharing mode.

In the virtual wireless network, each iteration of Algorithm 4 operates in a slot. At the end of each iteration, namely, the beginning of each slot, $\hat{x}^{t}$ will be selected as the resource sharing mode. It is easy to recognize from Algorithm 4 that the sequence of states visited $x^{t}$ is a Markov chain on the state space $\Omega$. Even the states visited are not guaranteed to converge, we will show in the following that under a weak condition, the sequence of $\hat{x}^{t}$ converges almost surely to the global optimizer.

\section{Verification of Convergence of Algorithm 4}

Theorem 5.1. [75] For $\tilde{x} \neq x^{*}, x \neq x^{*}$, and independent observations of the corresponding objective function values, if

$$
\operatorname{Pr}\left\{f\left(\boldsymbol{H}^{t}, x^{*}\right)>f\left(\boldsymbol{H}^{t}, x^{t}\right)\right\}>\operatorname{Pr}\left\{f\left(\boldsymbol{H}^{t}, x^{t}\right)>f\left(\boldsymbol{H}^{t}, x^{*}\right)\right\},
$$




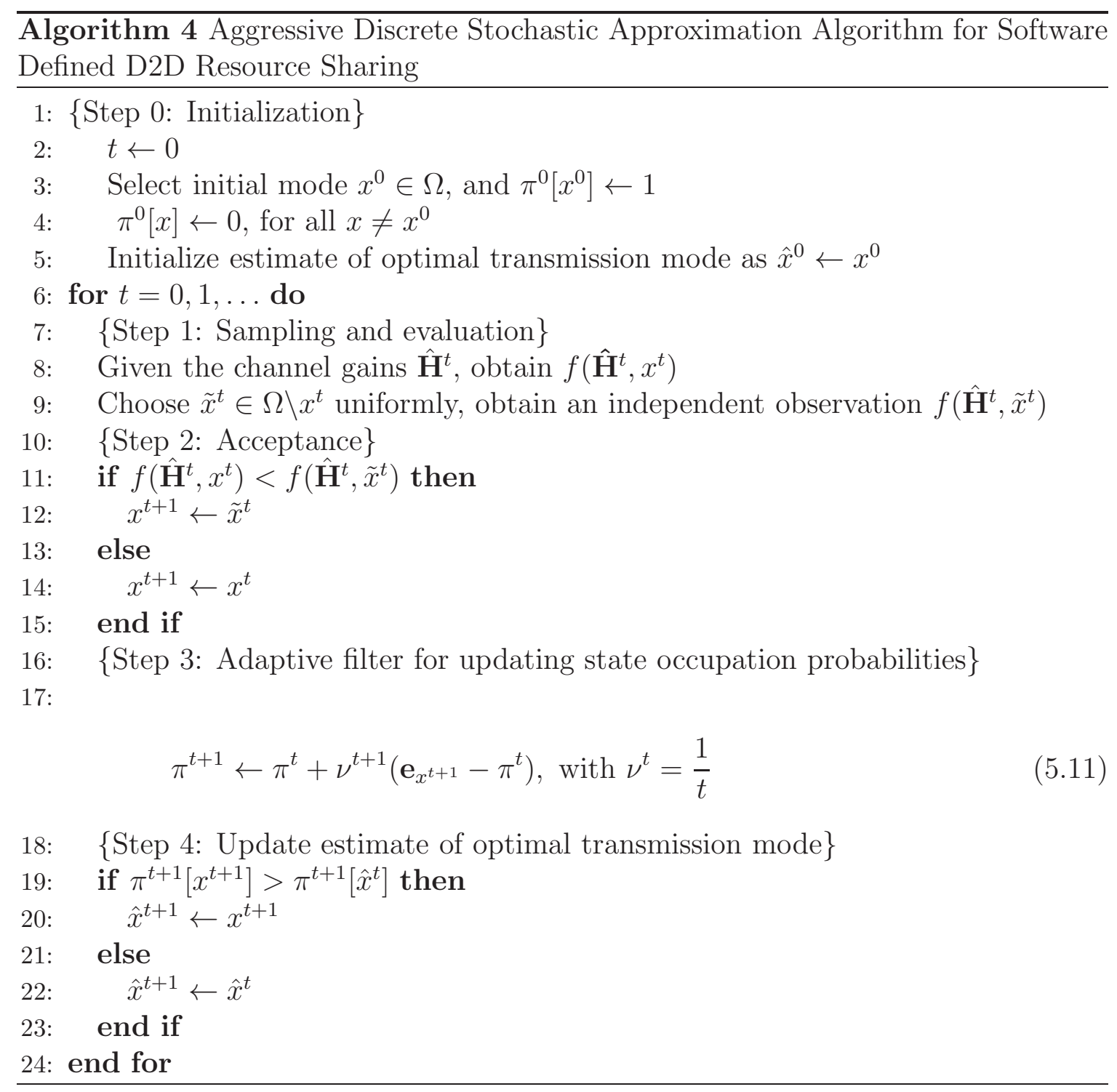




$$
\operatorname{Pr}\left\{f\left(\boldsymbol{H}^{t}, x^{*}\right)>f\left(\boldsymbol{H}^{t}, \tilde{x}^{t}\right\}>\operatorname{Pr}\left\{f\left(\boldsymbol{H}^{t}, x^{t}\right)>f\left(\boldsymbol{H}^{t}, \tilde{x}^{t}\right)\right\},\right.
$$

then the sequence $\left\{x^{t}\right\}$ is a homogeneous irreducible and aperiodic Markov chain with state space $\Omega$. Besides, for sufficiently large $t$, the sequence $\left\{\hat{x}^{t}\right\}$ spends more time in $x^{*}$ than any other states.

Using the similar techniques used in [79, 141], we verify that Algorithm 4 converges to the global optimizer after sufficient numbers of iterations. Assume the mean value and the variance of the objective $f\left(\mathbf{H}^{t}, x^{t}\right)$ are $\mathbb{E}\left[f\left(\mathbf{H}^{t}, x^{t}\right)\right]=\theta_{x}$ and $\operatorname{Var}\left(f\left(\mathbf{H}^{t}, x^{t}\right)\right)=$ $\sigma_{x}^{2}$, respectively. Consider the empirical accumulative distribution function $F_{n}(x)=$ $\frac{1}{N} \sum_{i=1}^{N} 1_{X_{i}<x}$ of $f\left(\mathbf{H}^{t}, x^{t}\right)$, in which random variable $X_{i}$ denotes the observations of the objective function. We can obtain empirical distributions of the objective function values. Fig. 5.4 is an example of the objective function values. The subplot on the left is the probability density function, and on the right the cumulative density function. By extensive simulations with MATLAB, we have verified that the distribution of the objective function can be approximated by a Gaussian distribution $N\left(\theta_{x}, \sigma_{x}^{2}\right)$ within a significance level of $5 \%$ based on Kolmogorov-Smirnov test [142] if the variance of the channel estimation error is less than or equal to $20 \%$ of the mean value of the actual channel gains.

Therefore, we can use Gaussian distribution to denote the distribution of the objective function given the channel estimation error is constrained within 20\%. Consider three different transmission subsets $x_{i}=x^{*}$ and $x_{j}, x_{l} \in\left\{\Omega \backslash x^{*}\right\}$. From the above, we have $f\left(\mathbf{H}^{t}, x_{i}^{t}\right) \sim \mathcal{N}\left(\theta_{x_{i}}, \sigma_{x_{i}}^{2}\right), f\left(\mathbf{H}^{t}, x_{j}^{t}\right) \sim \mathcal{N}\left(\theta_{x_{j}}, \sigma_{x_{j}}^{2}\right)$, and $f\left(\mathbf{H}^{t}, x_{l}^{t}\right) \sim \mathcal{N}\left(\theta_{x_{l}}, \sigma_{x_{l}}^{2}\right)$. Condition (6.18) can be rewritten as

$$
\operatorname{Pr}\left\{f\left(\mathbf{H}^{t}, x_{i}^{t}\right)-f\left(\mathbf{H}^{t}, x_{j}^{t}\right)>0\right\}>\operatorname{Pr}\left\{f\left(\mathbf{H}^{t}, x_{j}^{t}\right)-f\left(\mathbf{H}^{t}, x_{i}^{t}\right)>0\right\} .
$$

Due to the fact that samples of $f(\cdot)$ can be approximated by Gaussian distribution, 

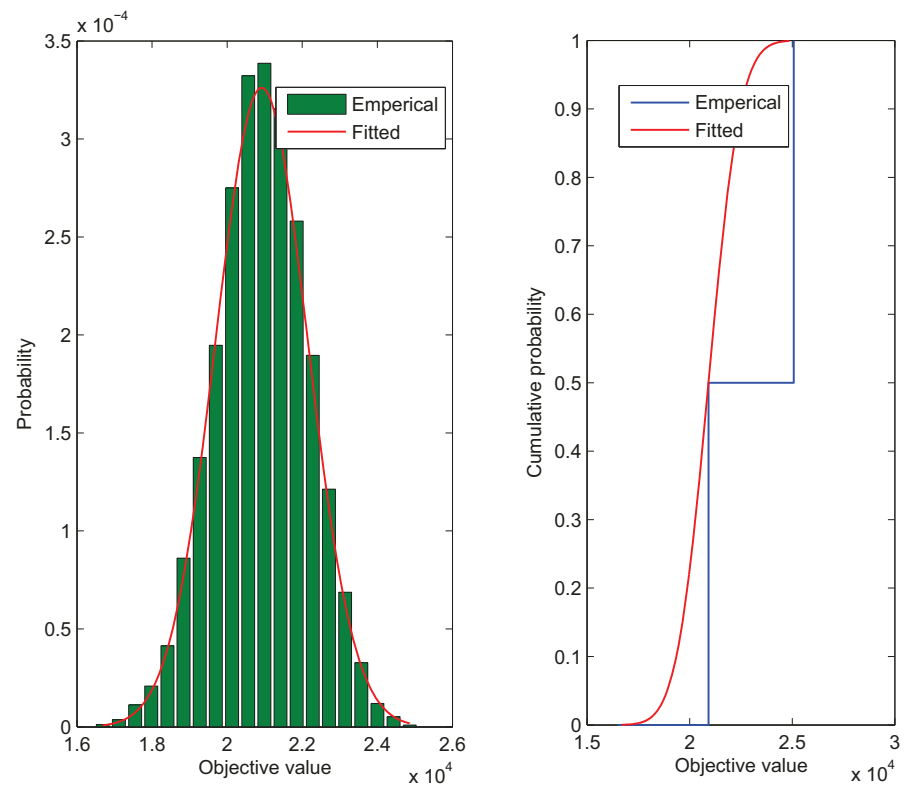

Figure 5.4: Statistical distributions of the sum user utility values based on channel estimates: CSI noise variance is equal to $5 \%$ of the mean channel gains.

the above inequality is equivalent to

$$
\operatorname{Pr}\left\{\mathcal{N}\left(\theta_{x_{i}}-\theta_{x_{j}}, \sigma_{x_{i}}^{2}+\sigma_{x_{j}}^{2}\right)>0\right\}>\operatorname{Pr}\left\{\mathcal{N}\left(\theta_{x_{j}}-\theta_{x_{i}}, \sigma_{x_{i}}^{2}+\sigma_{x_{j}}^{2}\right)>0\right\}
$$

Since $x_{i}=x^{*}$ is the maximizer of the problem, we have $\max \left\{\theta_{x_{i}}, \theta_{x_{j}}, \theta_{x_{k}}\right\}=x_{i}$; hence $\left(\theta_{x_{i}}-\theta_{x_{j}}\right)>\theta_{x_{j}}-\theta_{x_{i}}$. Therefore, (6.21) holds since both terms have the same variance.

Based on the Gaussian distribution approximation, (6.19) can be rewritten as

$$
\operatorname{Pr}\left\{\mathcal{N}\left(\theta_{x_{i}}-\theta_{x_{j}}, \sigma_{x_{i}}^{2}+\sigma_{x_{j}}^{2}\right)>0\right\}>\operatorname{Pr}\left\{\mathcal{N}\left(\theta_{x_{l}}-\theta_{x_{j}}, \sigma_{x_{l}}^{2}+\sigma_{x_{j}}^{2}\right)>0\right\}
$$

Furthermore it is equivalent to

$$
\frac{\theta_{x_{i}}-\theta_{x_{j}}}{\sqrt{\sigma_{x_{i}}^{2}+\sigma_{x_{j}}^{2}}}>\frac{\theta_{x_{l}}-\theta_{x_{j}}}{\sqrt{\sigma_{x_{l}}^{2}+\sigma_{x_{j}}^{2}}} .
$$


Again we verify the above inequality by extensive simulations.

\section{Adaptive Step Size for Resource Sharing Optimization in Time-Varying Channels}

In the previous section, Algorithm 4 solves the resource sharing optimization problem in static wireless channels, namely, the channel gains are fixed. However, the wireless channel in practical systems is time-varying. In Algorithm 4, the step size is set as $1 / t$, which makes the algorithm more and more conservative to stay at the most promising state as the algorithm proceeds. And the region of $\Omega$ are not sufficiently explored. In time-varying networks, the algorithm might stick to some suboptimal solution while getting more and more conservative. Therefore, $\nu^{t}$ has a prominent impact on the performance of the stochastic approximation algorithm. Intuitively, we should have $0 \leq \nu^{t} \leq 1$ to keep (6.16) as a probability. Consider two extreme cases. If $\nu=1$, the previous states are all forgotten, which is equivalent to the exhaustive search; if $\nu=0$, the state occupation probability is fixed at one value, which will miss the chance to find a better transmission subset.

To track the optimal step size $\nu^{*}$ in time-varying channels, we use an adaptive continuous least-mean-square (LMS) algorithm for the transmission optimization. Similar algorithm has been employed in [79] for MIMO antenna selection and in [140] for CDMA code optimization. Denote state occupation probability depending on the value of $\nu$ at iteration $t$ as $\pi^{\nu, t}$ and its mean-square derivative $(\partial / \partial \nu) \pi^{\nu, t}$ as $\mathbf{J}^{\nu, t}$, i.e.,

$$
\lim _{\Delta \longrightarrow 0} \mathbb{E}\left\{\left|\frac{\pi^{\nu+\Delta, t}-\pi^{\nu, t}}{\Delta}-\mathbf{J}^{\nu, t}\right|^{2}\right\}=0
$$

The error related to $\nu$ in iteration $t$ is denoted as

$$
\epsilon^{\nu, t}=\mathbf{e}_{x^{t+1}}-\pi^{\nu, t}
$$


Differentiating (6.16) with respect to $\nu$, we have

$$
\mathbf{J}^{\nu, t+1}=(1-\nu) \mathbf{J}^{\nu, t}+\left(\mathbf{e}_{x^{t+1}}-\pi^{\nu, t}\right) .
$$

Algorithm 5 is a modification of Algorithm 4. It adjusts the step size according to the error in (6.25) to minimize its expectation. In Algorithm 5, $\eta$ is called the learning

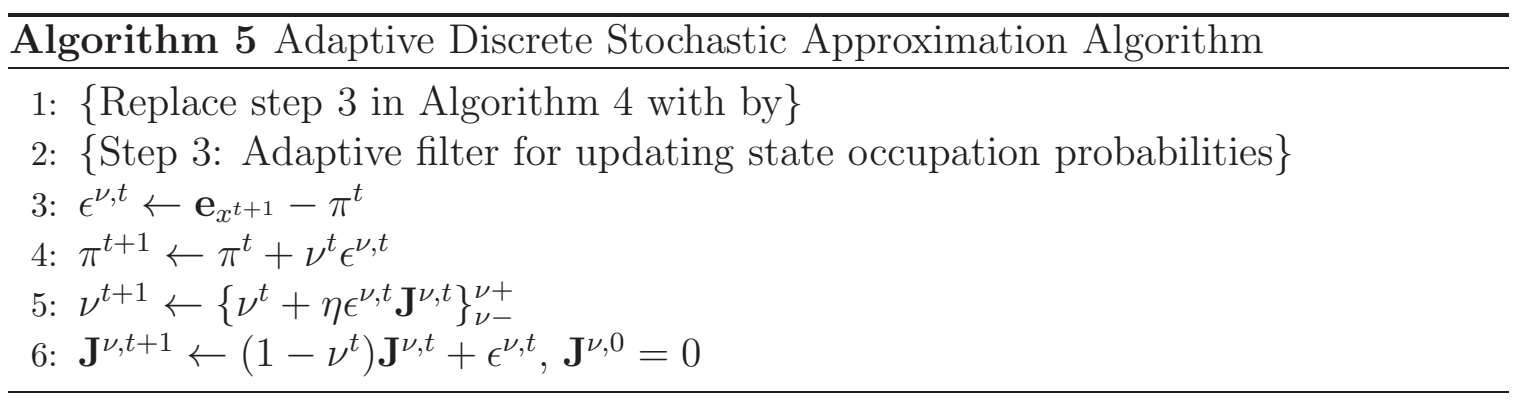

rate. If we set $\eta=0$, the step size is constant. With $0 \leq \nu^{-}<\nu^{+} \leq 1,\{X\}_{\nu^{-}}^{\nu^{+}}$denotes the projection of $X$ into $\left[\nu^{-}, \nu^{+}\right]$, i.e., $\{X\}_{\nu^{-}}^{\nu^{+}}=\min \left\{\max \left\{X, \nu^{-}\right\}, \nu^{+}\right\}$.

\subsubsection{Stochastic Traffic Arrivals and Imperfect QSI}

With random delay in transmitting QSI, we can only have noisy observation of the queue states, denoted as $\hat{\mathbf{Q}}$. ${ }^{4}$ The optimization problem in (5.7) is updated as,

$$
\underset{x \in \Omega}{\operatorname{maximize}} \mathbb{E}\left[f\left(\mathbf{Q}^{t}, \mathbf{H}^{t}, x^{t}\right)\right]
$$

We apply the discrete stochastic approximation algorithms developed above to this problem. Even it is intractable to show the convergence of the algorithms provided stochastic traffic and QSI delay, as we shall show in the following, we still can see considerable performance gains from our approach.

\footnotetext{
${ }^{4}$ The modeling of imperfectness of QSI is not as straightforward as CSI noise because the queuing state values are integers.
} 
Discussion: In this work, we assume unlimited buffer size. That means the latency are not considered in the formulation. However, this framework could be still applicable when limited buffer size is considered. For example, we can introduce the Lyapunov function [143] in the objective function (5.21).

\subsection{Simulation Results and Discussions}

In the simulations, we investigate a time-slotted system consisting of 7 access points and 300 users. There are in total 150 pairs of potential peer-to-peer requests, with one flow for each pair. The inter-site distance is 500 meters; the users are uniformly dropped in regions 300 meters away from their home access points. There are two InPs, denoted as $\operatorname{In} P_{1}$ and $\operatorname{In} P_{2}$. The default prices for $\operatorname{In} P_{1}, \operatorname{In} P_{2}$, and $D 2 D$ are $0.8 \$, 1.2 \$$, and $0.4 \$$, respectively. A single unit of radio resource of a bandwidth of $150 \mathrm{KHz}$ is assumed. The transmission power is fixed at $0 \mathrm{dBm}$. The noise power in $\mathrm{dBm}$ is computed as $-174+10 \log _{10}$ (Bandwidth). The wireless channel samples are generated based on SCME channel model [144], in which the carrier frequency is 2.11 GHz, user velocity $6 \mathrm{Km} / \mathrm{h}$, sample duration $1 \mathrm{~ms}$. The fading of the channels are assumed to be block fading with a block size of 100. For stochastic traffic arrivals, Poisson arrivals with a parameter $\lambda$ are used, in which the size of packets is 512 Bytes. The achievable link data rate is computed based on Shannon bound. The CSI noise is modeled as a zero-mean Gaussian random variable with the value of standard deviation being $5 \%$ of mean channel gain by default. To model QSI noise, we add Gaussian noise to actual queue lengths and truncate them into non-negative values. The standard deviation of the added Gaussian noise is also $5 \%$ of the average queue length by default.

In the following, we shall present the simulation results for various settings. The first two subsections discuss the performance under various traffic intensities and 
different prices. Then we shall discuss the convergence property of the proposed algorithms. At the end we present some results to illustrate the effect of different levels of NSI noise.

\subsubsection{Various Traffic Arrival Intensities}

To show the effect of traffic arrival intensity $\lambda$, we range $\lambda$ from 0 to 150 packets per second. Four schemes are compared. The Perfect NSI is the one exhaustively searching the best resource sharing decision given perfect NSI. The DSA is the one using Algorithm 5, for which the parameters used in the simulations are as follows: learning rate $\eta$ is 0.9 , lower bound and upper bound for $\nu$ are 0 and 0.9 , respectively. In the $w / o$ Virtualization scheme, the radio resources are shared among cells in a round-robin fashion, and either D2D or relay mode can be selected to utilize the resource but the resource sharing is constrained within a cell. To see the effect of data roaming, we assume half of the users need to roam to the $\mathrm{InP}$ they do not belong to. When roaming happens, an extra amount of money will be charged, which is the same as the price of the visited InP's price. The $w / o$ D2D scheme is the one having wireless network virtualization but D2D communication is disabled. For all the schemes except for $D S A$, perfect NSI is used to compute the results. This provides an upper bound for the baseline schemes including Perfect NSI, w/o Virtualizatio, and $w / o$ D2D since only imperfect NSI is available in practical networks.

To see the throughput performance, we need to bypass the effect of pricing. So we set the prices for all the resource utilization schemes to be $1.2 \$$. Since the DSA scheme belongs to random search, we run the algorithm for 100 times and plot the average performance in the figure. As shown in Fig. 5.5, with the increase of the traffic arrival, the achievable system throughput of all the schemes will saturate. As for the proposed DSA-based scheme, it can utilize imperfect NSI and its performance gap 


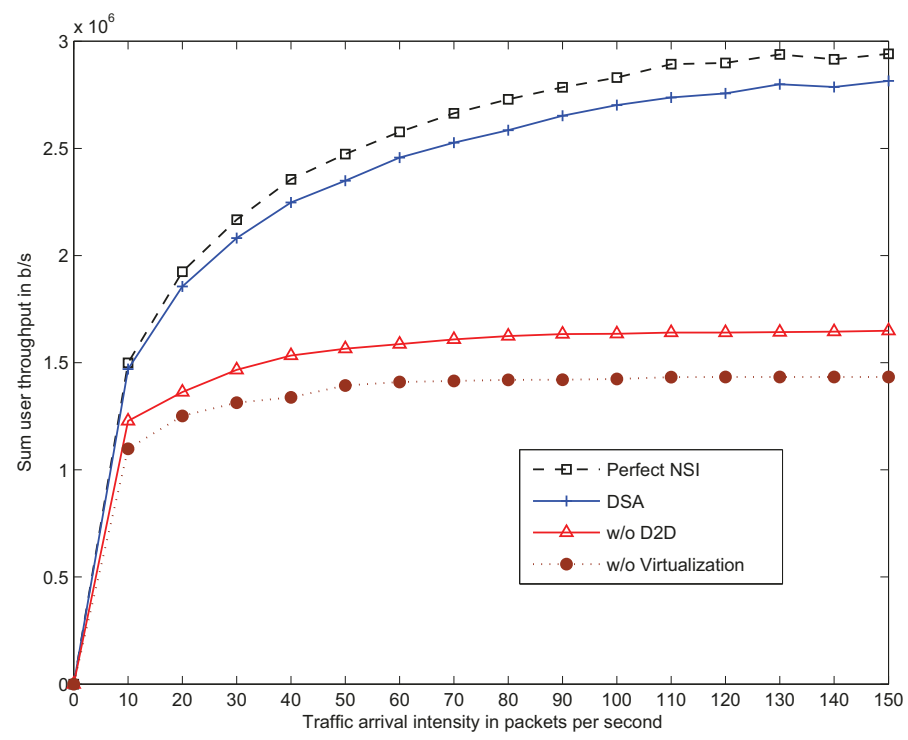

Figure 5.5: Sum user throughput performance vs. traffic arrival intensity.

between the Perfect NSI scheme is rather small. While for the two scheme utilizing merely either D2D or virtualization, the former one outperforms the later one in terms of system throughput.

Note that the higher system throughput cannot always bring more satisfaction to the users especially when the prices for the resource sharing schemes are different. Fig. 5.6 shows the sum user utilities, in which the prices for D2D, In $P_{1}$, and $\operatorname{In} P_{2}$ are $0.4 \$, 0.8 \$$, and $1.2 \$$, respectively. We can see that the DSA scheme can have close performance as the Perfect NSI scheme. However, the scheme without virtualization outperforms the one without D2D in terms of sum user utility in $b / s / \$$. Such contrast in the two figures is mainly caused by the price setting. We shall discuss the effect of prices in Sect. 5.5.2.

\subsubsection{Effect of Prices}

Fig. 5.7 and Fig. 5.8 show the sum user utility under different D2D prices provided the traffic arrival being 20 packets per second and the same NSI noise setting as 


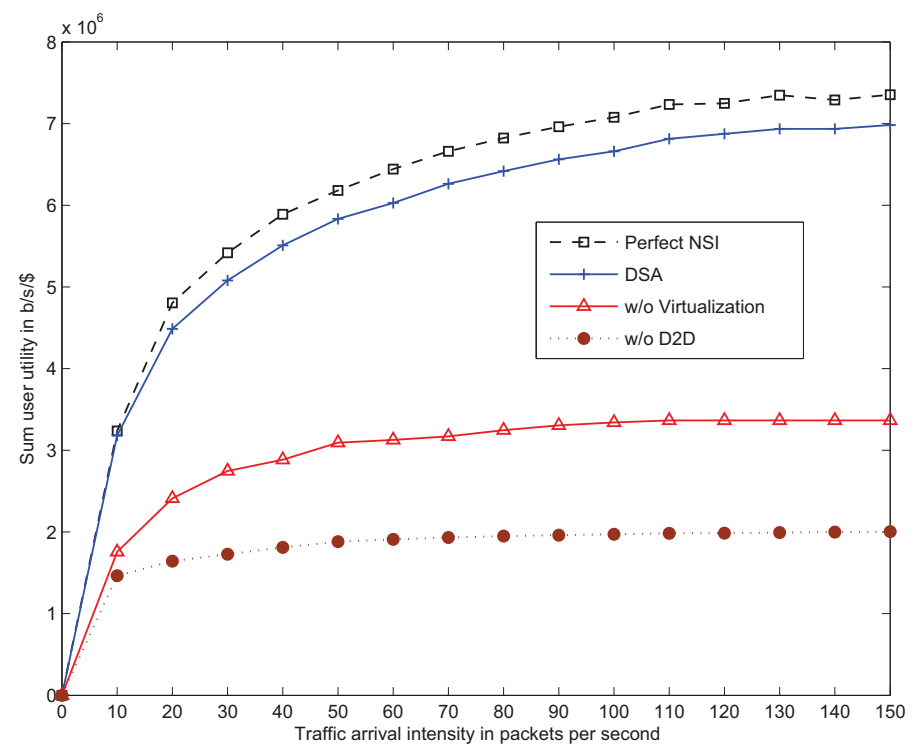

Figure 5.6: Sum user utility performance vs. traffic arrival intensity.

in Sect. 5.5.1. In terms of sum user utility, when the ratios of the D2D price and the two InPs' prices are greater than 1, the difference between the four schemes is marginal. That means the benefits of virtualization and D2D will be weakened as the price of $\mathrm{D} 2 \mathrm{D}$ increases. The scheme without $\mathrm{D} 2 \mathrm{D}$ is independent of the D2D price. In contrast, the scheme without virtualization is sensitive to the D2D prices. In particular, when the ratio is greater than about $0.875 \%$, the scheme without D2D has slightly higher sum utility than the scheme without virtualization.

Fig. 5.9 illustrates the effect of the ratio between $I n P_{1}$ 's and $I n P_{2}$ 's prices. The scheme without D2D is the most sensitive one among the four schemes because the options among the users are limited inside the relay transmission mode. Without virtualization, each cell takes turns to utilize the resource. But since D2D is enabled, the users' utility drops slightly as the price of $\operatorname{In} P_{1}$ increases. For the schemes employing both D2D and virtualization, the effect of a single InP's price is very marginal, since the resource sharing can be flexibly alternating between D2D and any InP bringing higher benefit to users. 


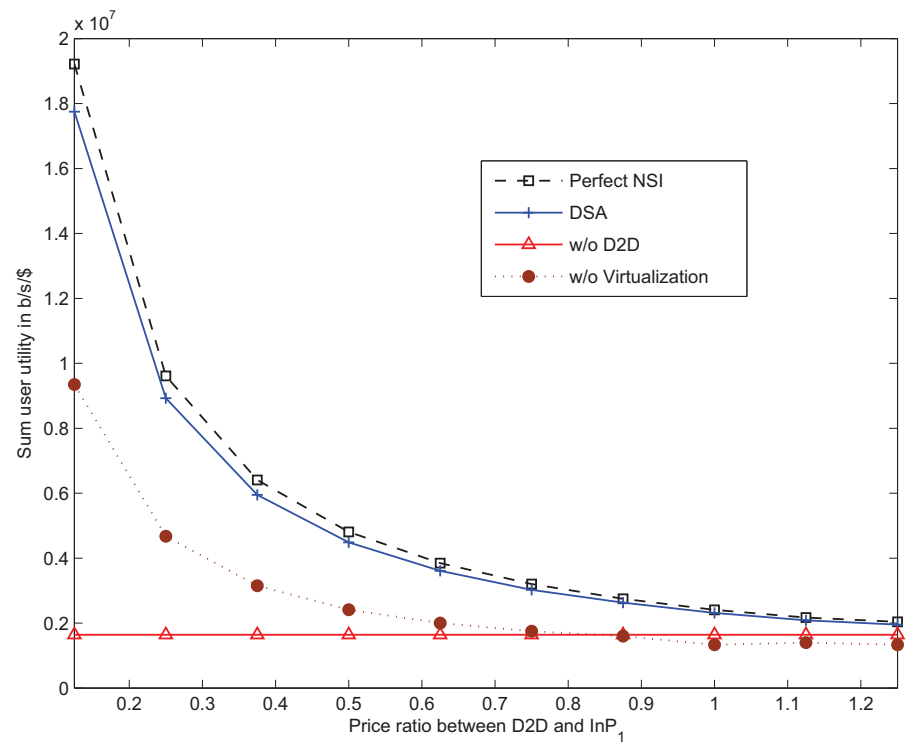

Figure 5.7: Sum user utility performance vs. the price ratio between D2D and $\operatorname{In} P_{1}$.

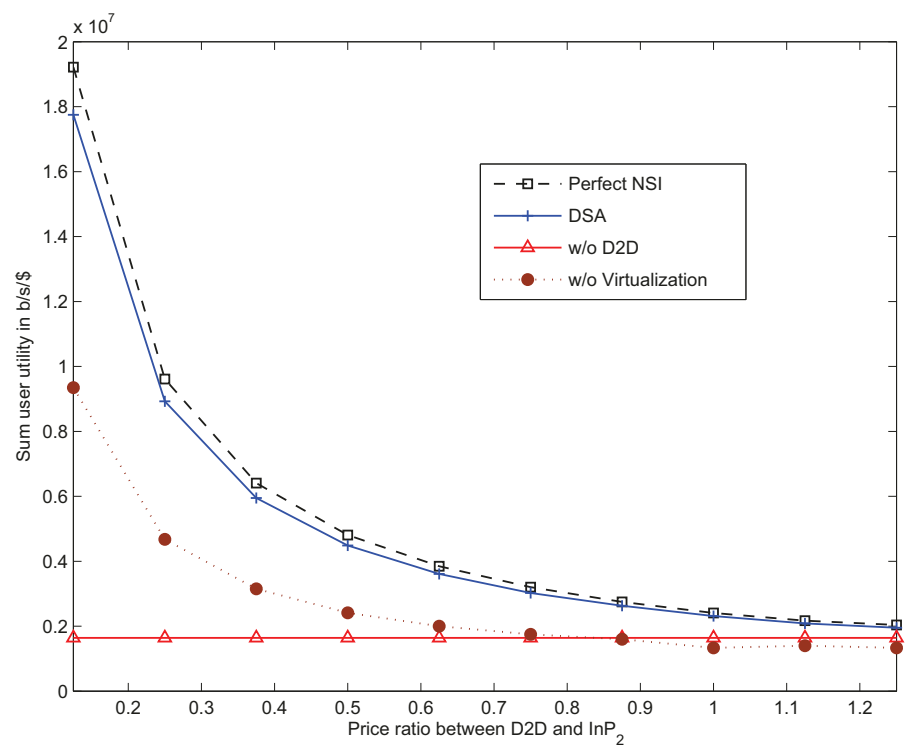

Figure 5.8: Sum user utility performance vs. the price ratio between D2D and $\operatorname{In} P_{2}$.

\subsubsection{Convergence Properties}

\section{Static Channel and Full Buffer Traffic}

To show the convergence property, we first investigate the case with static channels and full-buffer traffic discussed in Sect. 5.4.1, in which the inaccuracy of NSI merely 


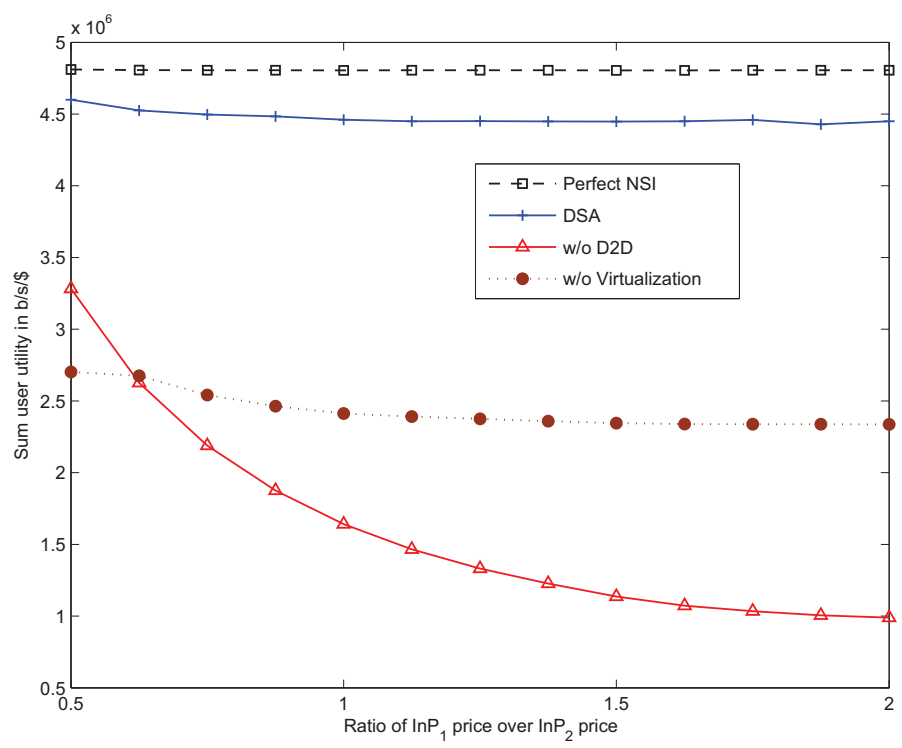

Figure 5.9: Sum user utility performance vs. the price ratio between $\operatorname{In} P_{1}$ and $\operatorname{In} P_{2}$.

comes from noisy CSI. Fig. 5.10 illustrates the achieved sum user utility with the standard deviation of CSI noise being $5 \%$ of the mean value of channel gains. There are two curves for DSA, the solid curve is the results averaged over 100 of iterations while the dotted one is one shot of the algorithm. Clearly we can see that the DSA algorithm is able to approach the optimal as the number of iteration increases. Such learning ability corresponds to the discussion in Sect. 5.4.1.

\section{Time-Varying Channel and Full Buffer Traffic}

An instance of sum user utility under time-varying channel and full-buffer traffic is demonstrated in Fig. 5.11. The CSI noise level is the same as in Sect. 5.5.3. Due to the block fading channel, the performances of the schemes except for DSA tend to be the static inside a block. While the proposed DSA-based scheme is able to approach to the optimal performance within a fading block. 


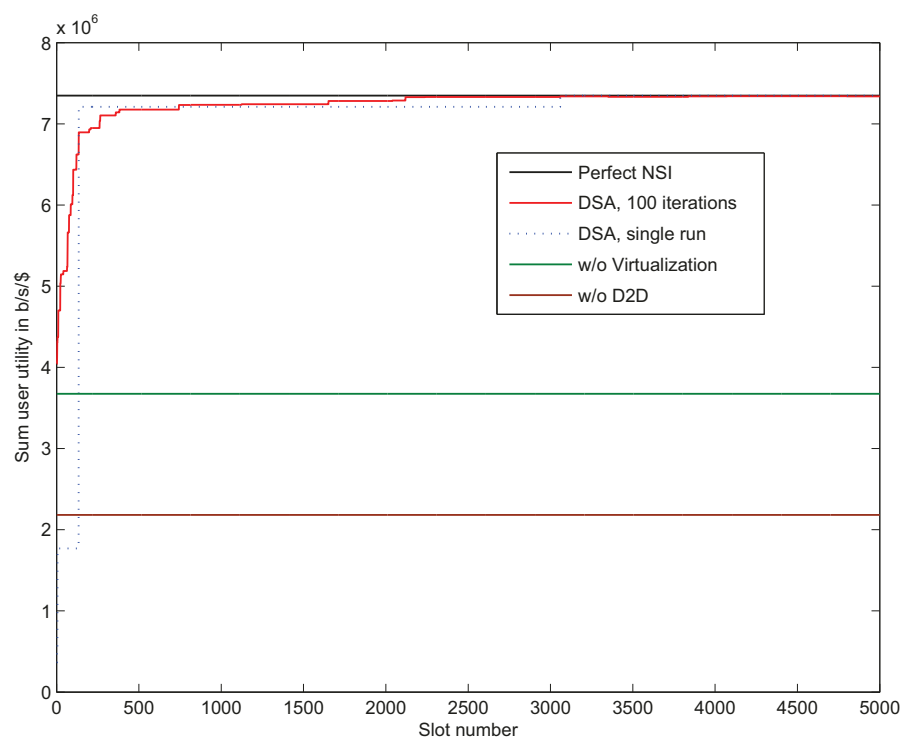

Figure 5.10: Sum user utility under static channels.

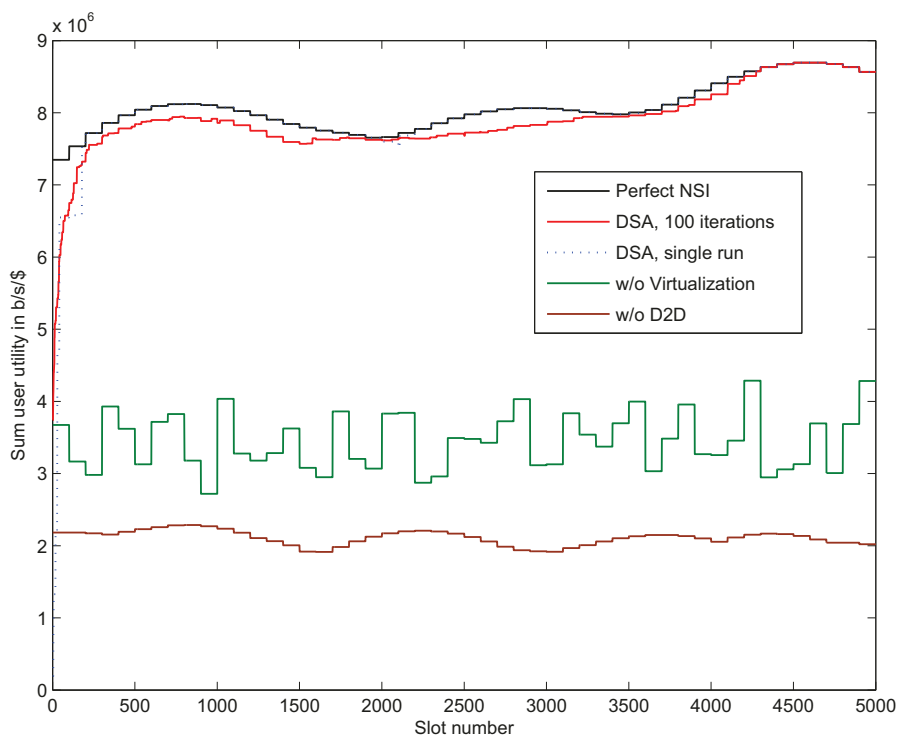

Figure 5.11: Sum user utility under time-varying channel: low CSI noise.

\subsubsection{Effect of NSI Noise}

In Fig. 5.12, we compare the cumulative density of the sum utility with low and high CSI noise in the full-buffer traffic scenario. The low and high CSI represent the standard deviation of CSI noise being $5 \%$ and $20 \%$ of the mean, respectively. In most 


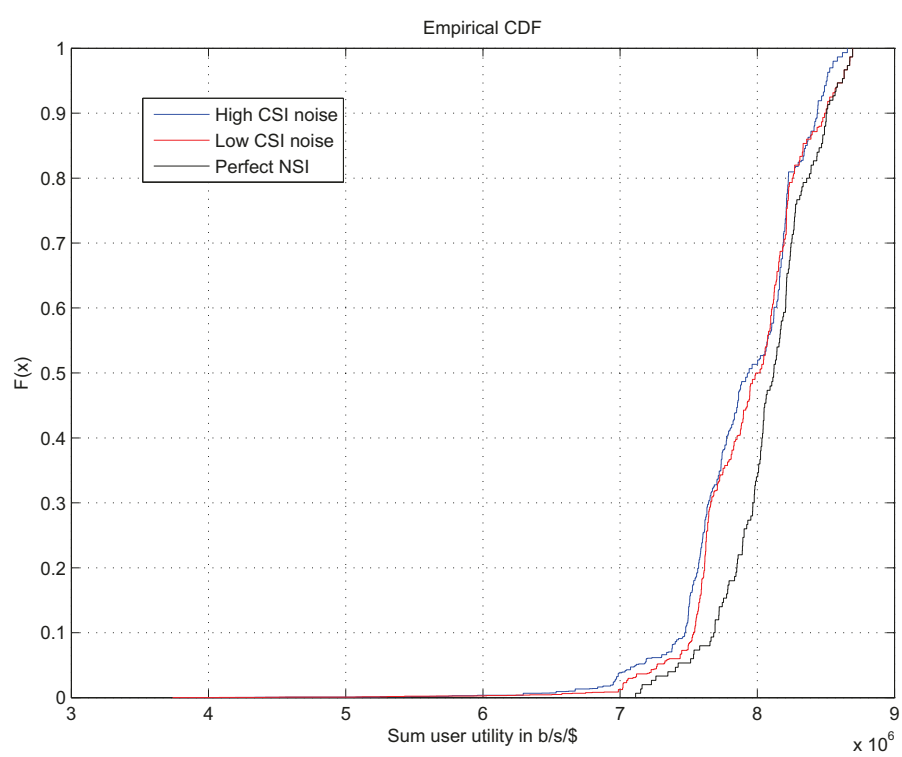

Figure 5.12: Cumulative density function of sum user utility (full-buffer traffic and noisy CSI).

cases, the DSA algorithm performs better given low CSI noise than high CSI noise. But the difference is still marginal. This implies that the proposed DSA algorithm is robust against CSI noise.

We fix the CSI noise at 5\% to see the effect of QSI noise. We first add Gaussian noise to the actual queue length values, and then round them into integers to represent noisy QSI values. For the low and high QSI noise curves in Fig. 5.13, the standard deviation of the Gaussian noise are $5 \%$ and $20 \%$ of $\lambda$, respectively. The results in Fig. 5.13 are under traffic intensity $\lambda$ of 20 . We see the given noisy CSI and noisy QSI, the DSA algorithm still shows satisfactory performance. Besides, with high QSI noise, its performance decades very slightly. Another observation from comparing Fig. 5.12, and 5.13 is that the noise in CSI has higher impact on DSA's performance than that in QSI. 


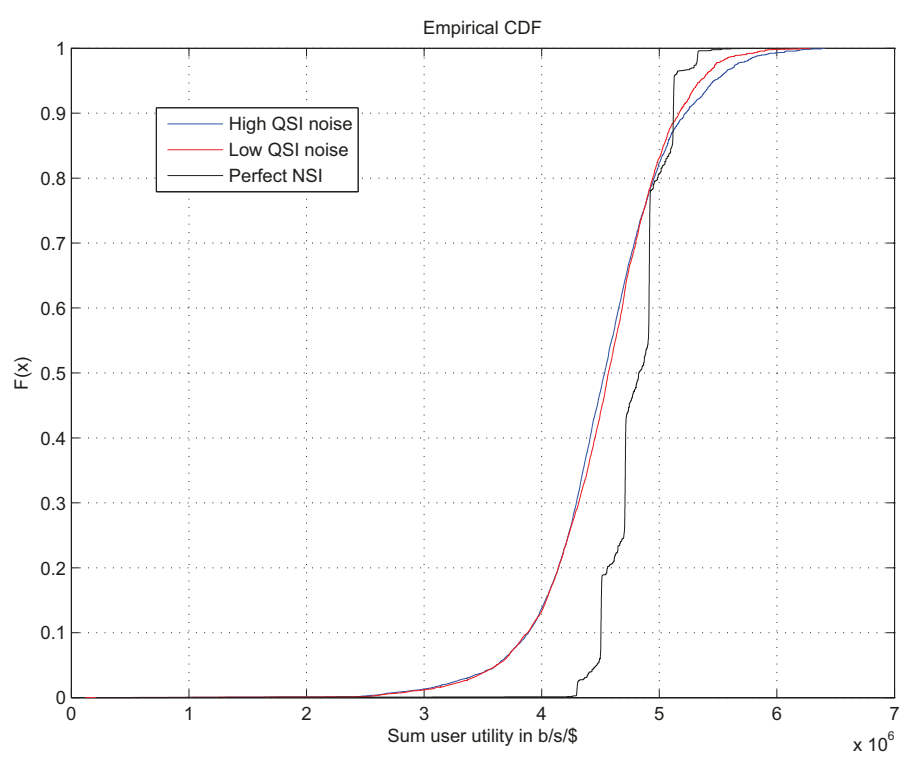

Figure 5.13: Cumulative density function of sum user utility (Poisson arrivals, noisy CSI and QSI).

\subsection{Chapter Summary}

In this work, we studied a framework to provide software defined D2D communications in virtual wireless networks. To address the challenge introduced by imperfect network state information, including channel state information and queuing state information, in this framework, we proposed resource sharing algorithms based on recent advances in discrete stochastic approximation. Both static channels and timevarying channels were considered. Extensive simulation results were presented to show that wireless mobile users can benefit from both wireless network virtualization and software defined D2D communications, and the proposed scheme can achieve significant performance gain under practical network settings. 


\section{Chapter 6}

\section{Scheduling for UAV Ad-Hoc Networks with FDR and MPR}

\subsection{Introduction}

Recently, the use of unmanned aerial vehicles (UAVs) in military and civilian applications is gaining more and more interests due to their unique sensing capabilities and emergency response efficiency for rescue operations in the regions where it is difficult or dangerous for human beings to access [145]. Communications and networking systems are crucial technologies for multiple UAV operations, particularly UAVs that operate in a collaborative and autonomous manner. In such operations, multiple UAVs need to dynamically establish an ad-hoc network without necessarily using a fixed infrastructure. Certainly, issues in traditional mobile ad-hoc networks (MANETs) in general still exist in UAV ad-hoc networks and need to be investigated. However, a UAV ad-hoc network has some distinct characteristics, such as high node mobility and delay-sensitive applications (e.g., battlefield applications), which introduce new and non-trivial challenges [146].

Although the existing MAC schemes in general improve the quality of service (QoS) in UAV ad-hoc networks, most of them are designed for traditional physical 
layer techniques, such as half-duplex radios without multi-packet reception capability. Indeed, in wireless communications, it is generally assumed that radios cannot receive and transmit on the same frequency band simultaneously because of the interference [147]. Therefore, most radio systems either use time to alternate transmitting and receiving, or use two separate frequencies, one for transmission and the other for reception. However, recent advances in the development of physical layer interference cancellation techniques have shown promise of full-duplex radios, where a wireless node can transmit and receive at the same time on a single channel [148-150]. In addition, most traditional MAC schemes are based on an idealized collision channel, in which if there is a single packet transmitted, it can be received without error; if there are more than one transmission, all the packets involved are corrupted due to a collision. With multi-packet reception, however, this assumption is no longer necessary, and it is possible to correctly receive multiple packets even when concurrent transmissions occur [151]. The full-duplex radios and multi-packet reception capability have significant impacts on the MAC performance [148, 151].

In this part, we study the media access control problem in UAV ad-hoc networks with full-duplex radios and multi-packet reception capability. The novelties of this work can be summarized as follows.

- To the best of our knowledge, this is the first research work to consider fullduplex radio and multi-packet reception in MAC designs for UAV ad-hoc networks.

- The case with only estimated CSI is considered for the MAC scheme for UAV ad-hoc networks. It is known that one of the most important parameters at physical layer is the channel state information (CSI). This information has been assumed to be perfectly known in most exiting MAC schemes for UAV ad-hoc networks. However, in practice, it is difficult to have the perfect knowledge 
of a dynamic channel $[152,153]$, especially in battlefields where interference, jamming and high mobility of nodes may cause the channel state to change dynamically.

- The MAC schemes in the presence of perfect and imperfect CSI are formulated as a combinatorial and a discrete stochastic optimization problem, respectively. Both system throughput and packet delay are considered in the optimization objectives.

- A token-based technique is proposed for updating information in the network. This technique is able to overcome the drawback of contention-based MAC schemes and efficiently handle the highly mobile environment in UAV ad-hoc networks. In wireless networks with strict latency requirements, a token-based protocol has shown its effectiveness and advantages over contention-based MAC $[64,154]$. We adapt the token-based protocol to include full-duplex radios and multi-packet reception capability, which is to enhance the MAC performance of UAV ad-hoc networks in highly mobile environments.

- We show, using computer simulation, that the use of physical layer full-duplex radios and multi-packet reception capability have significant impacts on the performance of UAV ad-hoc networks. In addition, we show that throughput and packet delay can be improved substantially in the proposed MAC scheme.

This chapter is organized as follows. Section 6.2 presents the system models. The problem of transmission optimization with perfect channel knowledge is formulated in Section 6.3. Section 6.4 discusses the transmission optimization with imperfect channel knowledge, and optimization algorithms are proposed to solve the problem. Simulation results are discussed in Section 6.5. Finally, we conclude this study in Section 6.5.5. 


\subsection{System Models}

In this section, we describe the system models including full-duplex radios and multipacket reception used in this chapter. The proposed token-based MAC protocol is also presented here.

\subsubsection{Full-Duplex Radios (FDRs)}

In wireless communications, it is generally assumed that a radio working on a single channel cannot transmit and receive at the same time (i.e., only half-duplex communication is allowed in a single channel). However, recent advances in the development of physical layer interference cancellation techniques have shown promise of full-duplex radios for wireless communications [148-150]. A full-duplex communication system based on the software defined radio is reported in [149], where analogue interference cancellation and a nulling transmit antenna are used. In [150], the authors presented a full-duplex communication system using commercial off-the-shelf multiple-input and multiple-output (MIMO) radios. Their experiments show that, by using a different mix of analogue and digital cancellation techniques, full-duplex communication is feasible in many cases. A full-duplex radio in a single channel is proposed in [148] in a practical way, in which three self-interference cancellation schemes, i.e.,, antenna cancellation, RF (radio frequency) interference cancellation, and digital cancellation, are combined to implement an IEEE 802.15.4 full-duplex radio.

Since the full-duplex technology is expected to change the fundamental assumptions in wireless communications, designing upper layer protocols and algorithms to exploit this new physical layer technology for improving system-wide performance becomes a highly desirable necessity.

With full-duplex communications, many problems in wireless networks, such as hidden terminal problems, could be potentially mitigated. In this chapter, we explore 
the benefits of full-duplex communications for media access control. In networks with half-duplex communications, if there is a packet coming when a node is transmitting, the packet will be corrupted. This cases is called primary collision. To avoid primary collisions, there are many proposals to schedule the wireless nodes in either the transmitting or the receiving states [155]. However, even with perfect scheduling, there is only one node that is allowed to transmitt in a slot for a pair of wireless nodes. This increases the communication latency for both nodes. With full-duplex wireless communications, the delay incurred can be reduced since each node is able to transmit and receive at the same time.

Nevertheless, in practice, even with advanced self-interference cancellation technologies, we cannot ignore the effect of transmitting power on the reception in the same node. To incorporate the self-interference cancellation capability, similar to [156], we introduce a parameter, the self-interference cancellation factor $\psi$, to represent the effect of self-interference cancellation from the transmit power. Assuming that the transmit power of a full-duplex node $n$ is $P_{n}$, with concurrent transmission and reception, the self-interference on the reception is equal to $\psi P_{n}$.

\subsubsection{Multi-Packet Reception (MPR)}

With multi-packet reception (MPR) capability, a receiver is able to receive more than one packet correctly when multiple nodes transmit packets to a common receiver simultaneously. The MPR capability can be described by a matrix [157], in which each element denotes the probability that the number of packets are correctly received out of the number of simultaneous transmissions. In a wireless network with MPR capability, this matrix can be constructed using channel statistics that include the effects of multi-path fading, node mobility, and multi-user interference. As an example of MPR modeling, we assume in this study that each node employs a code division 
multiple access (CDMA) transceiver with a matched filter for receiving. Note that our scheme can also be used in a network using other physical layer techniques with the MPR capability.

We use in our formulation the signal-to-interference-plus-noise ratio (SINR) threshold reception model [158]. Suppose that a set of wireless nodes, $\mathbf{S}=$ $\{1,2, \ldots, n-1, n\}$, have data to send in a slot. In the set, node $n$ is assumed to receive data from node 1 to $n-1$. For node $n$ itself, its potential receiver is node $n-1$, as shown in Fig. 6.1. Consider the transmission from node $i, i=1, \ldots, n-1$, to node $n$ in Fig. 6.1. Assume that the channel gain and transmission power for node $i$ are $g_{i}$ and $P_{i}$, respectively, the background noise power is $\sigma^{2}$, and the spreading factor is $N$. According to the SINR threshold reception model, if the received SINR is greater than a threshold $\beta$, i.e.,

$$
S I N R \simeq \frac{P_{i} g_{i}}{\sigma^{2}+\frac{1}{N} \sum_{k=1, k \neq i}^{n-1} g_{k} P_{k}}>\beta,
$$

then the packet from $i$ can be received correctly. Combining the MPR capability with full-duplex radios, the SINR threshold reception model becomes

$$
S I N R \simeq \frac{P_{i} g_{i}}{\sigma^{2}+\frac{1}{N} \sum_{k=1, k \neq i}^{n-1} g_{k} P_{k}+\psi P_{n}}>\beta
$$

\section{Token-based MAC}

In a traditional wire-line token ring protocol [159], the token travels along the nodes in a determined sequence and a node can transmit data only when it holds the token. The token is released and passed to another node once the node finishes transmission, 


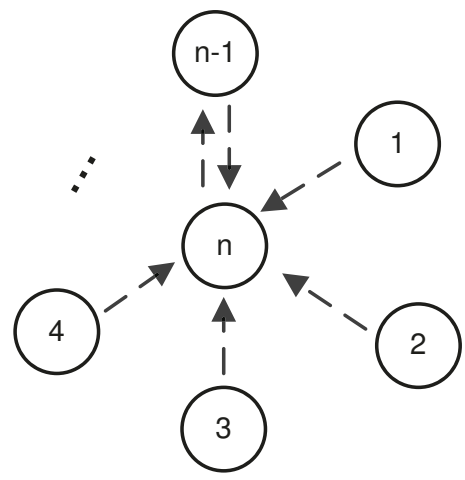

Figure 6.1: A transmission set.

\begin{tabular}{|c|c|c|}
\hline \multicolumn{2}{|c|}{ Token Passing } & Common Channe \\
\hline Data A & Data D & \multirow[t]{3}{*}{$\rightarrow$ Data Channel } \\
\hline Data B & Data E & \\
\hline Data C & Data F & \\
\hline
\end{tabular}

Figure 6.2: The media access control framework.

\begin{tabular}{|l|l|l|}
\hline Code list & Channel gain list & Delay requirement list \\
\hline
\end{tabular}

Figure 6.3: The structure of the token.

or once it has transmitted for a maximum allowed time. There are also some tokenring protocols for wireless networks. The Wireless Token Ring Protocol (WTRP) [64] is a modification of the token-based protocol to provide guaranteed delay and bandwidth in MANETs. It is shown that WTRP is able to recover from link failures due to wireless nodes' mobility. Liu et al. considered the effects of the CDMA physical layer technology on MAC designs in [65]. In their proposal, there is a list of available CDMA codes for a receiver in the token. However, full-duplex radios are not considered in [65].

To meet the QoS requirements for UAV ad-hoc networks, we propose the following token-based protocol in our MAC scheme. As shown in Fig. 6.2, there is a token passing in the common channel, and data frames are transmitted in the data channels. 
In UAV networks, the connectivity of wireless links may change rapidly due to UAVs' high mobility. Therefore, holding a token while transmitting could cause latency and a waste of wireless resources if the token holder or its receiver happens to move away from the token ring. Hence, in our token-based MAC, when a node with data to send holds a token, it will take a code out of the code list in the token, and pass the token to the next node immediately.

The structure of a token is illustrated in Fig. 6.3. The code list consists of the codes of all nodes in the ring to receive data frames. Here we assume the number of codes is greater than the number of transmitters. The channel gain list contains the channel gain of each link in the token ring. In the delay requirements list, there are the normalized amounts of time that the transmitters have been waiting. When a token is passed to a node, for instance, node $i$, and if node $i$ has data to send to another node, say, node $n$, it will take the code of node $n$ from the code list. In addition, it will update the channel gains it measured and the time that the data packet has been waiting in node $i$ 's queue. The channel gains and the delay requirements in the token will be used for transmission optimization described in Sections 6.3 and 6.4.

To summarize, this chapter studies the design of a token-based MAC for UAV ad-hoc networks with full-duplex radios and MPR capability. When several nodes have data to send to a common receiver, they know, with the help of token passing, the channel gains of each transmitter and the corresponding amounts of time that the transmitters have been waiting. Based on the available information, each node finds the optimal action to ensure that the QoS requirements are met. The MAC optimization problems with perfect and imperfect CSI are formulated in Section 6.3 and Section 6.4, respectively. 


\subsection{Transmission Optimization with Perfect Channel Knowledge}

In the MAC protocol presented above, a critical issue is which nodes should be scheduled for transmissions, given the channel state information and delay requirements obtained from token passing. In other words, in each slot, every node that has data to transmit needs to decide whether or not to send a data frame, based on the channel states and the delay requirements. Specifically, transmitter $i$ chooses an action $x_{i}$ from its action set $\{0,1\}$, where 0 represents Not Transmit, and 1 represents Transmit.

$$
x_{i} \in\{0,1\}, i=1,2, \ldots, n
$$

Let $\mathbf{x}$ denote the actions taken by all the $n$ nodes.

$$
\mathbf{x}=\left[x_{1}, x_{2}, \ldots, x_{i}, \ldots, x_{n}\right]^{T} \in\{0,1\}^{n \times 1} .
$$

Note that if the node $n$ in Fig. 6.1 does not have data to send, which is a classical half-duplex communication scenario, we can simply set $x_{n}=0$. So the model we use here is a general case for all possible scenarios. The notations that we use in our formulation are summarized in Table 6.1.

\subsubsection{Constraints}

To fully exploit the MPR capability and full-duplex radios, each transmission needs to satisfy (6.2). Suppose the transmit power of node $i(i=1,2, \ldots, n-1)$ is $P_{i}$ and 
CHAPTER 6. SCHEDULING FOR UAV AD-HOC NETWORKS WITH FDR AND MPR124

Table 6.1: Notation Summary in Chapter 6

\begin{tabular}{|c||c|}
\hline Notation & Definition \\
\hline $\mathbf{x}$ & The vector of decision variables of all nodes \\
$x_{i}$ & Decision variable of node $i$ \\
$P_{i}$ & The transmission signal power of node $i$ \\
$g_{i}$ & Channel gain of node $i$ to its receiver \\
$\psi$ & Self-interference cancellation factor \\
$\sigma^{2}$ & The power density of background noise \\
$\beta$ & SINR threshold \\
$d_{i}$ & The amount of time that node $i$ has been waiting \\
\hline
\end{tabular}

its channel gain is $g_{i}$, the SINR threshold condition for node $i$ is formulated as

$$
\frac{g_{i} P_{i}}{\sigma^{2}+\frac{1}{N} \sum_{k=1, k \neq i}^{n-1} g_{k} P_{k} x_{k}+\psi P_{n} x_{n}}>\beta, \text { if } x_{i}=1 .
$$

Following the $k$-Fold Alternative approach in [160], we can manipulate the above constraint as

$$
B x_{i}+\frac{\beta}{N} \sum_{k=1, k \neq i}^{n-1} g_{k} P_{k} x_{k}+\beta \psi P_{n} x_{n}<g_{i} P_{i}+B-\beta \sigma^{2}
$$

where $B$ is a large positive integer such that the inequality holds when $x_{i}=0$, namely, when the SINR constraint is no longer needed.

For node $n$ in Fig. 6.1, the SINR threshold is

$$
\frac{g_{n} P_{n}}{\sigma^{2}+\psi P_{n-1} x_{n-1}}>\beta, \text { if } x_{n}=1
$$


Using the same approach, it is reformulated as

$$
\beta \psi P_{n-1} x_{n-1}+B x_{n}<g_{n} P_{n}+B-\beta \sigma^{2} .
$$

The $n$ constraints guarantee that the received SINR of nodes that decide to transmit is greater than the threshold, so that the transmissions will be successful.

For ease of presentation, we assume that each node uses the same transmission power, i.e., $P_{i}=P$, for $i=1,2, \ldots, n$. In fact, if we take power control into consideration, we only need to adapt the transmission power as variables. Denote the coefficients matrix in (6.6) and (6.8) as $A$, and denote the vector composed by the number to the right hand side of the inequalities as $\mathbf{b}$. The constraints can be described as

$$
A \mathbf{x} \prec \mathbf{b}, \mathbf{x} \in\{0,1\}^{n \times 1},
$$

where $\prec$ represents the component-wise $<$ between two vectors [161].

\subsubsection{Objective}

Our objectives of the media access control design include to maximize the system throughput and to minimize the delay each node-pair withstands. With the help of token passing, all the nodes have the knowledge of the channel states and the delay information of the other nodes. Therefore, each node is able to make a decision to optimize the system-wide performance without any coordination from a central node. Specifically, each node needs to solve two optimization problems to maximize the system's throughput and minimize the delay.

Let's consider the system throughput first. According to Shannon's channel capacity formula, with better SINR, a higher data rate can be achieved given a spectrum 
bandwidth. In practical systems employing adaptive modulation and coding, when the SINR is better, a higher order of modulation and coding scheme will be adopted, which will result in higher data rates and hence higher throughput.

Recall that the SINR is expressed as (6.5) and (6.7). Therefore, the system wide throughput maximization problem can be described as

$$
\begin{array}{ll}
\underset{\mathbf{x}}{\operatorname{maximize}} & T P(\mathbf{x}) \\
\text { subject to } & A \mathbf{x} \prec \mathbf{b}, \mathbf{x} \in\{0,1\}^{n \times 1},
\end{array}
$$

where

$$
\begin{aligned}
& T P(\mathbf{x}) \\
= & \sum_{i=1}^{n-1} \log \left(1+\frac{g_{i} P}{\sigma^{2}+\frac{1}{N} \sum_{k=1, k \neq i}^{n-1} g_{k} P x_{k}+\psi P x_{n}}\right) x_{i}+\log \left(1+\frac{g_{n} P}{\sigma^{2}+\psi P x_{n-1}}\right) x_{n} .
\end{aligned}
$$

Let $d_{i}$ be the time that node $i$ has been waiting. To minimize the delay, we need to first schedule the nodes that have been waiting for the longest time. Mathematically, node $i$ needs to solve the following optimization problem

$$
\begin{array}{ll}
\underset{\mathbf{x}}{\operatorname{maximize}} & D(\mathbf{x}) \\
\text { subject to } & A \mathbf{x} \prec \mathbf{b}, \mathbf{x} \in\{0,1\}^{n \times 1}
\end{array}
$$

where

$$
D(\mathbf{x})=\sum_{i=1}^{n} d_{i} x_{i}
$$

Note that (6.11) is a maximization problem since the parameter $d_{i}$ is the amount of time that node $i$ has been waiting. When $\mathbf{x}=[1,1, \ldots, 1]^{T}$ (i.e., all the nodes 
transmit), $D(\mathbf{x})$ is maximized; then the delay is decreased since none of the nodes needs to wait. When $\mathbf{x}=[0,0, \ldots, 0]^{T}$ (i.e., all the nodes do not transmit), $D(\mathbf{x})=0$; then the delay is increased since none of the nodes transmits in this slot.

\subsubsection{Delay-Throughput Trade-off}

Since we consider both system throughput and delay in the MAC design, the optimization problem is a multi-objective optimization problem mathematically, where there is a trade-off between throughput and delay. One approach to solve a multi-objective optimization problem is via scalarization [161]. So we introduce a parameter $\lambda$ to account for the trade-off between throughput and delay, and formulate the problem as

$$
\begin{array}{ll}
\underset{\mathbf{x}}{\operatorname{maximize}} & T P(\mathbf{x})+\lambda D(\mathbf{x}) \\
\text { subject to } & A \mathbf{x} \prec \mathbf{b}, \mathbf{x} \in\{0,1\}^{n \times 1},
\end{array}
$$

Note that the problem is a combinatorial optimization problem, which is NP-hard. However, since in UAV networks the number of UAVs is usually no more than 10 , we can solve this problem by brute-force search. On the other hand, when there are too many UAVs, to solve this problem efficiently, we can linearize the throughput in the objective function as

$$
\sum_{i=1}^{n} x_{i}\left[\log \left(1+\gamma_{i}\right)\right]
$$

where $\gamma_{i}$ denotes the SNR of node $i$, which does not include the interference from other nodes. Eq. (6.13) is reasonable because it favorites the nodes with good channel conditions. Finally, the problem can be formulated as the following binary programming 
problem.

$$
\begin{array}{ll}
\underset{\mathbf{x}}{\operatorname{maximize}} & \sum_{i=1}^{n} x_{i}\left[\log \left(1+\gamma_{i}\right)+d_{i} \lambda\right] \\
\text { subject to } & A \mathbf{x} \prec \mathbf{b}, \mathbf{x} \in\{0,1\}^{n \times 1} .
\end{array}
$$

This binary optimization problem can be solved by searching algorithms based on the Branch and Bound principle [160]. Or we can also use commercial solvers such as CPLEX [162] to solve it.

\subsection{Discrete Stochastic Optimization with Imper- fect Channel Knowledge}

In practical UAV ad-hoc networks, it is difficult to obtain perfect channel knowledge due to hardware limitation, channel estimation delay, etc. Instead, only the estimates of channel information can be obtained. So in the following we discuss the transmission optimization scheme based on imperfect channel knowledge.

\subsubsection{Discrete Stochastic Approximation Algorithm}

Denote the estimated channel gains at time slot $t$ as $\mathbf{g}^{(t)} \in \mathbb{R}^{n \times 1}$. Given a sequence of noisy estimates of channel gains, both the objective function and the constraints in (6.12) are stochastic. So the stochastic version of (6.12) is a constrained discrete stochastic optimization problem, where it is difficult to show the convergence of a solution [163]. So we first reformulate (6.12) into an unconstrained optimization problem. In (6.12), there are $n$ constraints, each of which represents the SINR threshold requirement for a successful transmission. In reality, when a transmission 
from node $i$ fails, the corresponding throughput and QoS utility for this node diminish. Therefore, given a transmission combination, when we evaluate the objective function, only those successful transmissions account for the value of the objective function. We denote this objective value as $f(\cdot)$. We can also interpret this scheme as an application of Lagrange Relaxation, in which the Lagrange multipliers for the feasible and infeasible transmissions are 1 and 0 , respectively.

Recall that the set of potential transmitters is $\mathbf{S}=\{1,2, \ldots, i, \ldots, n\}$. Here we denote the set of all possible transmitter subsets as $\Omega$, and each transmitter subset as $\omega \in \Omega$. Note that the number of possible transmitter subsets, $|\Omega|$, is equal to $2^{n}$. Therefore the problem we need to solve is to choose an $\omega$ out of $|\Omega|$ candidates such that the estimated objective value is maximized. Since the number of UAVs is generally less than 10, which is not a large number, and stochastic approximation algorithm is able to handle problems of this scale. For a specific $\omega$, given a sequence of unbiased channel estimates $\mathbf{g}^{(t)}$, the corresponding objective is a random variable denoted as $f\left(t, \omega^{(t)}\right)$. So the discrete stochastic optimization problem is to maximize the expected objective value, that is,

$$
\underset{\omega \in \Omega}{\operatorname{maximize}} \mathbb{E}\left[f\left(t, \omega^{(t)}\right)\right]
$$

In the following we adopt the discrete stochastic approximate algorithm $[75,79]$ to solve (6.15). Before we do that we introduce some symbols for notational simplicity. Denote a $|\Omega| \times 1$ vector with a one in the position decided by $\omega$ and zeros elsewhere as $\mathbf{e}_{\omega}$; such $|\Omega|$ unit vectors represent the $|\Omega|$ possible values of $\omega$.

Each iteration in Algorithm 6 is composed of 5 steps. In Step 1, a transmission set $\tilde{\omega}^{(t)}$ is uniformly chosen, and the corresponding objective value is evaluated. Here we assume the channel estimation technique in each iteration is unbiased. This is also the assumption used in [79]. We can achieve unbiased estimates by using different 


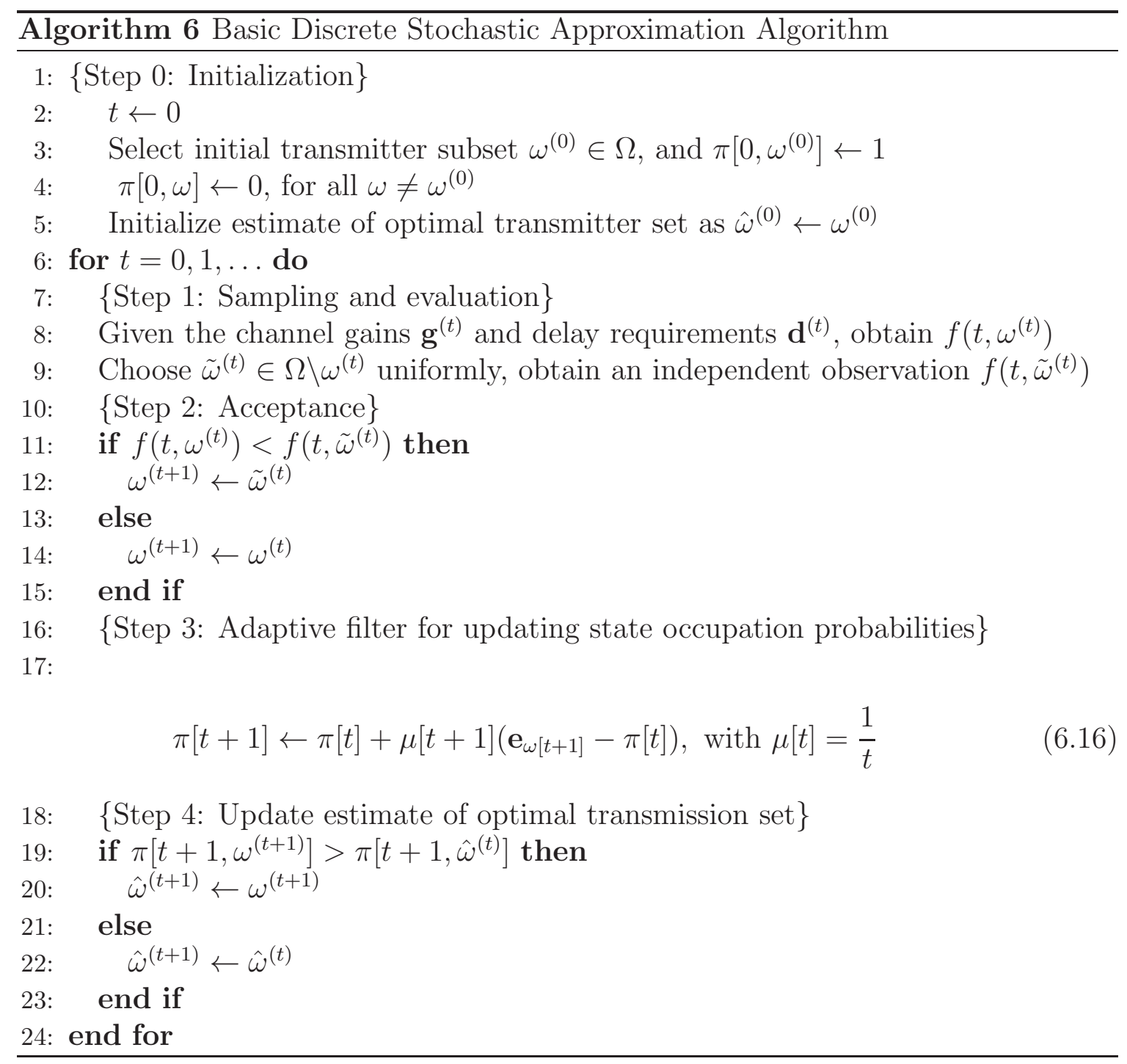


training preambles in channel estimation. If the randomly chosen objective value is larger than that of the current state $\omega^{(t)}$, it is accepted as the next visited state. In practical UAV ad-hoc networks, each iteration of Algorithm 6 operates in a slot. At the end of each iteration, namely, the beginning of each slot, $\hat{\omega}^{(t)}$ will be selected as the transmission set. The vector $\pi[t]$ records the relative frequency of each transmission subset that has been visited up to time $t$, i.e.,

$$
\pi\left[t, \omega^{(t)}\right]=\frac{\# \text { of visits to } \omega^{(t)}}{t}
$$

It is easy to recognize from Algorithm 6 that the sequence of states visited $\omega^{(t)}$ is a Markov chain on the state space $\Omega$. Even the states visited are not guaranteed to converge, we will show in the following that under a weak condition, the sequence of $\hat{\omega}^{(t)}$ converges almost surely to the global optimizer.

\subsubsection{Convergence Discussion}

According to [75], the sufficient condition for Algorithm 6 to converge is as follows. For $\tilde{\omega} \neq \omega^{*}, \omega \neq \omega^{*}$, and independent observations of the corresponding objective values, if

$$
\begin{aligned}
& \operatorname{Pr}\left\{f\left(t, \omega^{*}\right)>f(t, \omega)\right\}>\operatorname{Pr}\left\{f(t, \omega)>f\left(t, \omega^{*}\right)\right\}, \\
& \operatorname{Pr}\left\{f\left(t, \omega^{*}\right)>f(t, \tilde{\omega})\right\}>\operatorname{Pr}\{f(t, \omega)>f(t, \tilde{\omega})\},
\end{aligned}
$$

the sequence $\left\{\omega^{(t)}\right\}$ is a homogeneous irreducible and aperiodic Markov chain with state space $\Omega$, and the sequence $\left\{\hat{\omega}^{(t)}\right\}$ converges almost surely to $\omega^{*}$.

Using the similar techniques used in [79], we have the following theorem.

Theorem 6.1. (Global Convergence). Algorithm 6 converges to the global optimizer 
after sufficient iterations.

Proof. Assume the expected value and variance of the objective $f(t, \omega)$ are given by $\mathbb{E}[f(t, \omega)]=\mu_{\omega}$ and $\operatorname{Var}(f(t, \omega))=\sigma_{\omega}^{2}$, respectively. Consider the empirical accumulative distribution function $F_{n}(x)=\frac{1}{N} \sum_{i=1}^{N} 1_{X_{i}<x}$ of $f(t, \omega)$, in which random variable $X_{i}$ denotes the observations of the objective function. We can obtain empirical distributions of the objective values. Fig. 6.4 is an example of the objective values based on estimated channel knowledge in a network of 8 nodes. The subplot on the left is the probability density function, and on the right the cumulative density function. By extensive simulations with MATLAB, we have verified that the distribution of the objective function can be approximated by a Gaussian distribution $N\left(\mu_{\omega}, \sigma_{\omega}^{2}\right)$ within a significance level of $5 \%$ based on Kolmogorov-Smirnov test [142] if the expectation of the channel estimation error is less than or equal to $15 \%$.

Therefore, we can use Gaussian distribution to denote the distribution of the objective function given the channel estimation error is constrained within 15\%. Consider three different transmission subsets $\omega_{i}=\omega^{*}$ and $\omega_{j}, \omega_{l} \in\left\{\Omega \backslash \omega^{*}\right\}$. From the above, we have $f\left(t, \omega_{i}\right) \sim \mathcal{N}\left(\mu_{\omega_{i}}, \sigma_{\omega_{i}}^{2}\right), f\left(t, \omega_{j}\right) \sim \mathcal{N}\left(\mu_{\omega_{j}}, \sigma_{\omega_{j}}^{2}\right)$, and $f\left(t, \omega_{l}\right) \sim \mathcal{N}\left(\mu_{\omega_{l}}, \sigma_{\omega_{l}}^{2}\right)$. Condition (6.18) can be rewritten as

$$
\operatorname{Pr}\left\{f\left(t, \omega_{i}\right)-f\left(t, \omega_{j}\right)>0\right\}>\operatorname{Pr}\left\{f\left(t, \omega_{j}\right)-f\left(t, \omega_{i}\right)>0\right\} .
$$

Due to the fact that samples of $f(\cdot)$ can be approximated by Gaussian distribution, the above inequality is equivalent to

$$
\operatorname{Pr}\left\{\mathcal{N}\left(\mu_{\omega_{i}}-\mu_{\omega_{j}}, \sigma_{\omega_{i}}^{2}+\sigma_{\omega_{j}}^{2}\right)>0\right\}>\operatorname{Pr}\left\{\mathcal{N}\left(\mu_{\omega_{j}}-\mu_{\omega_{i}}, \sigma_{\omega_{i}}^{2}+\sigma_{\omega_{j}}^{2}\right)>0\right\}
$$

Since $\omega_{i}=\omega^{*}$ is the maximizer of the problem, we have $\max \left\{\mu_{\omega_{i}}, \mu_{\omega_{j}}, \mu_{\omega_{k}}\right\}=\omega_{i}$; hence $\left(\mu_{\omega_{i}}-\mu_{\omega_{j}}\right)>\mu_{\omega_{j}}-\mu_{\omega_{i}}$. Therefore, (6.21) holds since both terms have the same 

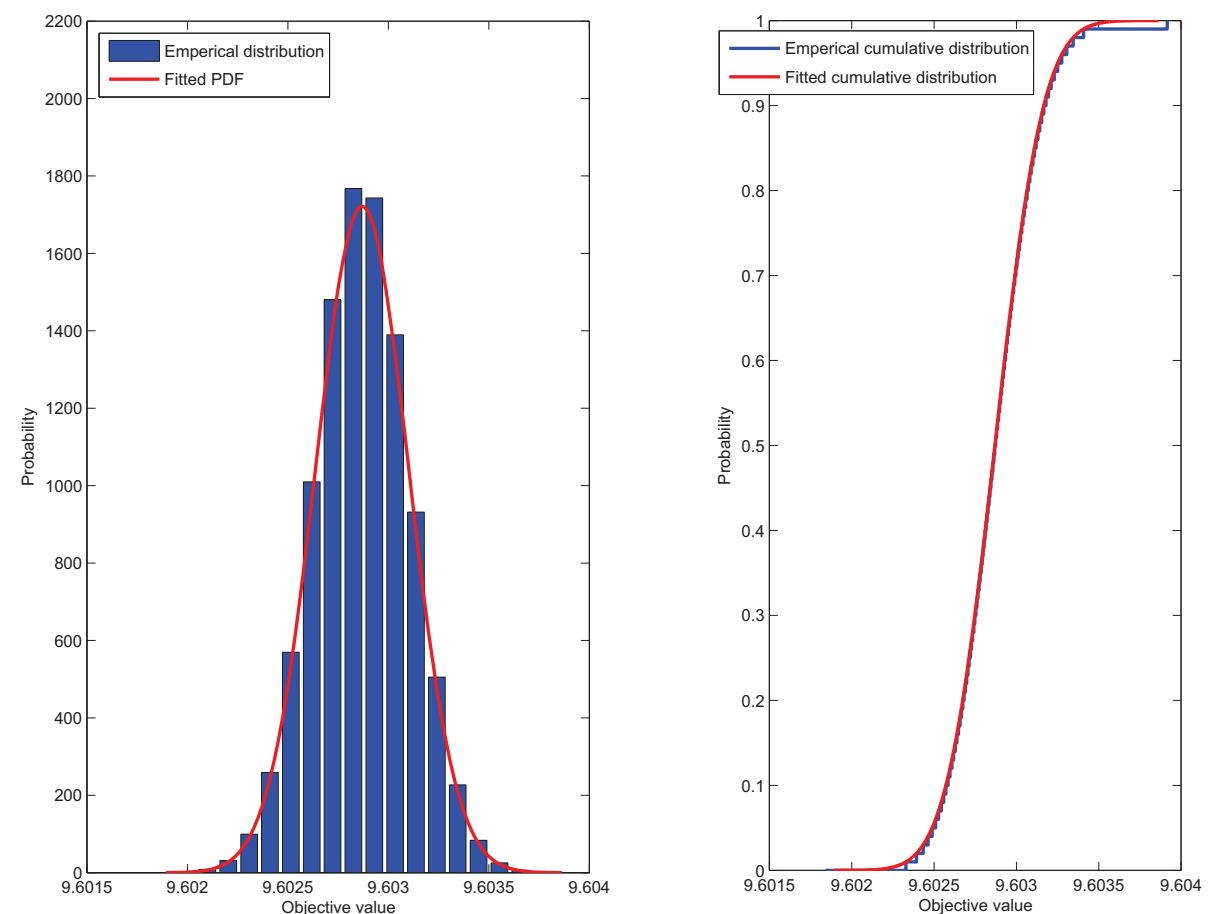

Figure 6.4: Statistical distributions of the objective value based on channel estimates.

variance.

Based on the Gaussian distribution approximation, (6.19) can be rewritten as

$$
\operatorname{Pr}\left\{\mathcal{N}\left(\mu_{\omega_{i}}-\mu_{\omega_{j}}, \sigma_{\omega_{i}}^{2}+\sigma_{\omega_{j}}^{2}\right)>0\right\}>\operatorname{Pr}\left\{\mathcal{N}\left(\mu_{\omega_{l}}-\mu_{\omega_{j}}, \sigma_{\omega_{l}}^{2}+\sigma_{\omega_{j}}^{2}\right)>0\right\}
$$

Furthermore it is equivalent to

$$
\frac{\mu_{\omega_{i}}-\mu_{\omega_{j}}}{\sqrt{\sigma_{\omega_{i}}^{2}+\sigma_{\omega_{j}}^{2}}}>\frac{\mu_{\omega_{l}}-\mu_{\omega_{j}}}{\sqrt{\sigma_{\omega_{l}}^{2}+\sigma_{\omega_{j}}^{2}}} .
$$

Again we verify the above inequality by extensive simulations. 


\subsubsection{Adaptive Step Size for Transmission Optimization in Time-Varying Channels}

In the previous section, Algorithm 6 solves the transmission optimization problem in static wireless channels, namely, the channel gains are fixed. However, the wireless channel in practical systems is time-varying. In fact, the value of the step size $\mu$ has a prominent impact on the performance of the stochastic approximation algorithm. Apparently we should have $0 \leq \mu \leq 1$ to keep (6.16) as a probability. Consider two extreme cases. If $\mu=1$, the previous states are all forgotten, which is equivalent to the exhaustive search; if $\mu=0$, the state occupation probability is fixed at one value, which will miss the chance to find a better transmission subset. In Algorithm 6, the step size is set as $1 / t$, which makes the algorithm more and more conservative to stay at the most promising state as the algorithm proceeds.

To track the optimal step size $\mu^{*}$ in time-varying channels, we use an adaptive continuous least-mean-square (LMS) algorithm for the transmission optimization. This algorithm has been employed in [79] for MIMO antenna selection and in [140] for CDMA code optimization. Denote state occupation probability depending on the value of $\mu$ as $\pi^{\mu}[t]$ and its mean-square derivative $(\partial / \partial \mu) \pi^{\mu}[t]$ as $\mathbf{J}^{\mu}[t]$, i.e.,

$$
\lim _{\Delta \rightarrow 0} \mathbb{E}\left\{\left|\frac{\pi^{\mu+\Delta}[t]-\pi^{\mu}[t]}{\Delta}-\mathbf{J}^{\mu}[t]\right|^{2}\right\}=0
$$

Define the error as

$$
\epsilon^{\mu}[t]=\mathbf{e}_{\omega[t+1]}-\pi^{\mu}[t]
$$

Differentiating (6.16) with respect to $\mu$, we have

$$
\mathbf{J}^{\mu}[t+1]=\mathbf{J}^{\mu}[t]-\mu \mathbf{J}^{\mu}[t]+\left(\mathbf{e}_{\omega[t+1]}-\pi^{\mu}[t]\right) .
$$


Algorithm 7 is a modification of Algorithm 6. It adjusts the step size according to the error in (6.25) to minimize its expectation. In Algorithm 7, $\eta$ is called the learning

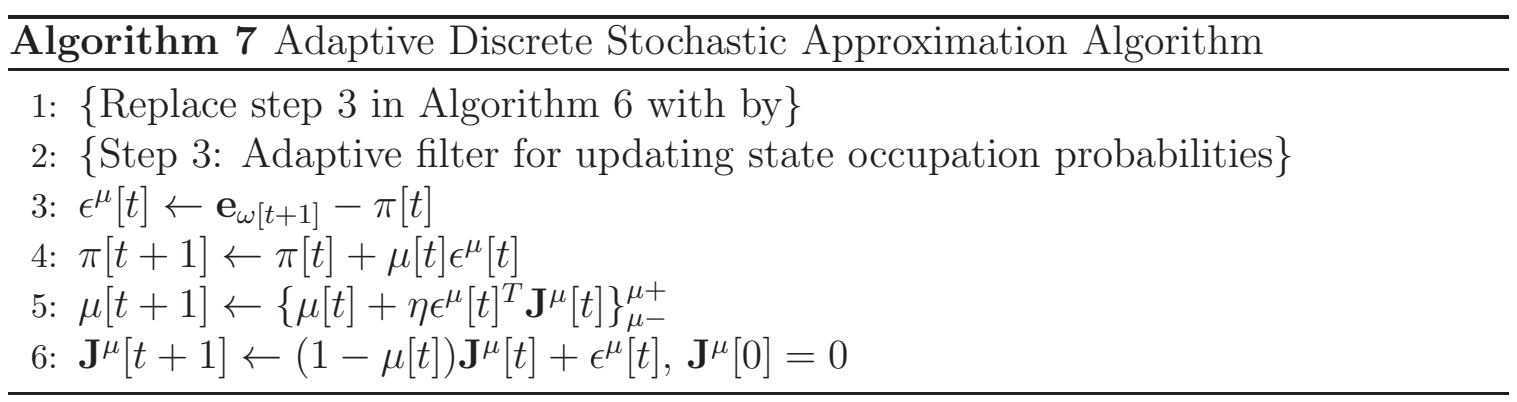

rate. If we set $\eta=0$, the step size is constant. With $0 \leq \mu^{-}<\mu^{+} \leq 1,\{X\}_{\mu^{-}}^{\mu^{+}}$ denotes the projection of $X$ into $\left[\mu^{-}, \mu^{+}\right]$, i.e., $\{X\}_{\mu^{-}}^{\mu^{+}}=\min \left\{\max \left\{X, \mu^{-}\right\}, \mu^{+}\right\}$.

\subsection{Simulation Results and Discussions}

In this section we evaluate our proposed MAC scheme via computer simulations with MATLAB. In the simulation we consider a UAV ad-hoc network where the UAVs move at an average speed of $100 \mathrm{Km} /$ hour in a square region of $10 \mathrm{Km} \times$ $10 \mathrm{Km}$ following the random way point model [164]. We assume that all UAVs are experiencing i.i.d. Rayleigh fading without spatial correlation. Hence the channel state (SNR) is modeled as an exponential distributed random variable with unit mean. Both perfect channel information and imperfect channel information are used in the simulations. The mean packet size is 512 Bytes. The parameters used in the simulations are listed in Table II.

We first investigate the performance again obtained via our scheme compared with the existing schemes in Subsection 6.5.1 where the channel estimation error rate is $5 \%$ when only imperfect CSI is available. In Subsection 6.5.2 and 6.5.3 we study the effect of two important physical layer parameters, namely, self-interference cancelation factor and channel estimation error. And in Subsection 6.5.4, we show 
Table 6.2: Simulation Parameters for UAV Ad-Hoc Networks

\begin{tabular}{|c||c|}
\hline Paramters & Value \\
\hline SINR threshold, $\beta$ & 3 \\
Transmit power & $40 \mathrm{dBm}$ \\
Spreading factor, $N$ & 32 \\
PHY header sized & $128 \mathrm{bits}$ \\
Modulation scheme & MQAM \\
Mean packet size & 512 Bytes \\
Channel & $2.4 \mathrm{GHz}$ \\
Carrier frequency & 3 \\
Path loss exponent & $1 \mathrm{dBi}$ \\
Antenna gain & Raleigh fading, unit mean \\
UAV mean speed & miles per hour \\
UAV mobility model & 100 way point \\
The learning rate, $\eta$ & $10^{-4}$ \\
$\mu^{-}$ & 0.0005 \\
$\mu^{+}$ & 0 \\
Thercentage of channel estimation error & 0.9 \\
\hline
\end{tabular}

the convergence of the stochastic approximation algorithms.

\subsubsection{Performance Improvement}

In Fig. 6.5 and Fig. 6.6, we compare the throughput and delay performance of our proposed schemes with that of existing token-based MAC schemes. Here, throughput is defined as the number of data bytes successfully received by the destination nodes per second, and delay is defined in a single hop sense since we only focus on link layer performance here. Existing token-based MAC schemes, including the traditional 


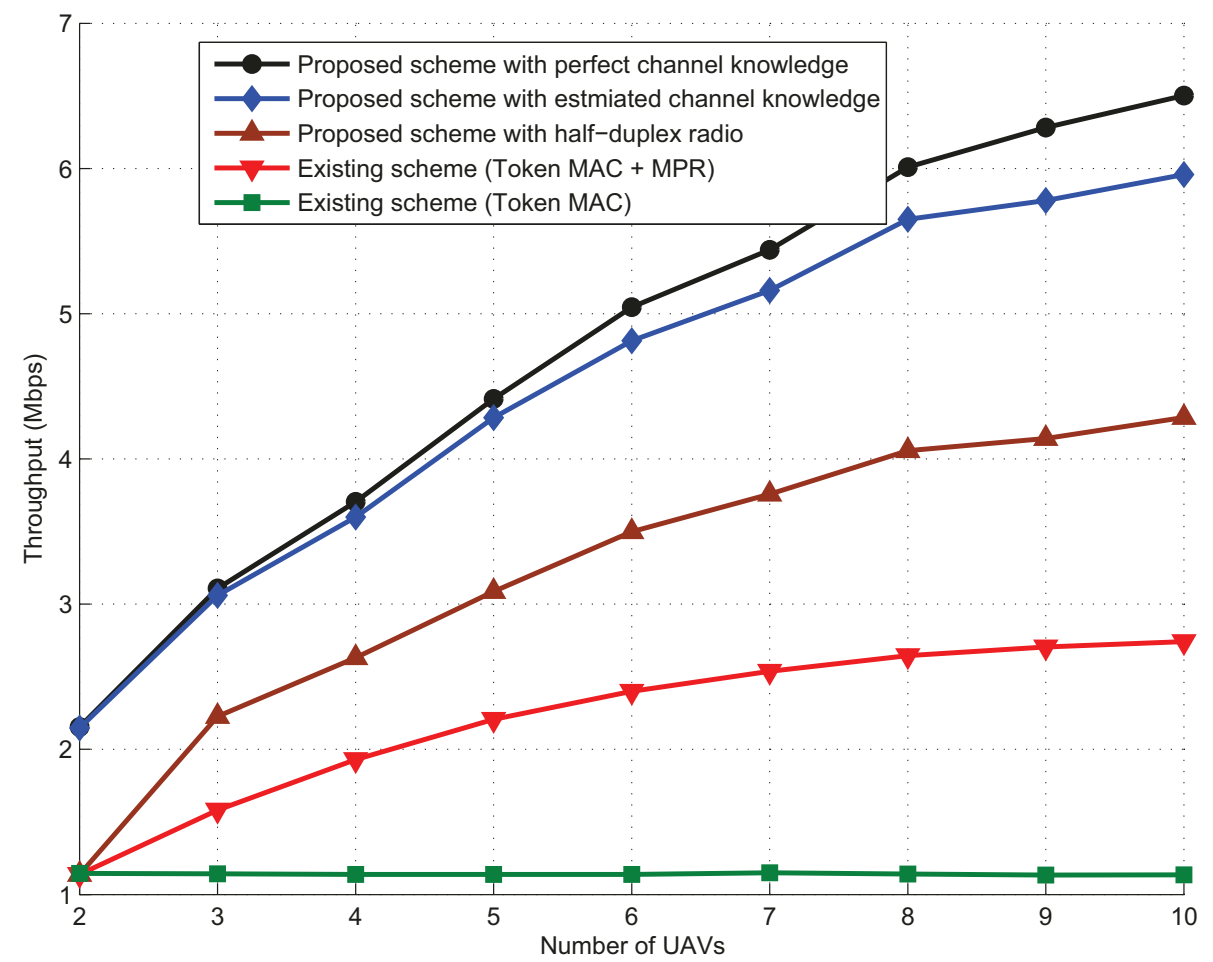

Figure 6.5: Throughput performance versus the number of UAVs.

token ring MAC [159] and the token-based MAC with MPR capability ${ }^{1}$ [65] are used for comparison. In traditional token ring MAC, neither full-duplex radios nor MPR is employed. the token-based MAC with MPR capability [65], full-duplex radios are not considered. To see the effect of full-duplex radios, we also investigate the case where our scheme works on a half-duplex radio network.

From these two figures, we observe that the proposed scheme with half-duplex radio outperforms the existing scheme (Token + MPR) with the help of the optimization based on channel state information. The performance is further increased in the proposed scheme when full-duplex radio is deployed, because more transmissions are allowed with full-duplex radios. Specifically, in the traditional token ring

\footnotetext{
${ }^{1}$ We omit the leaky bucket algorithm in [65] because here we only focus on the effect of physical layer technology on MAC layer; and the maximum number of CDMA codes, $M$ in [65], is set as 10.
} 


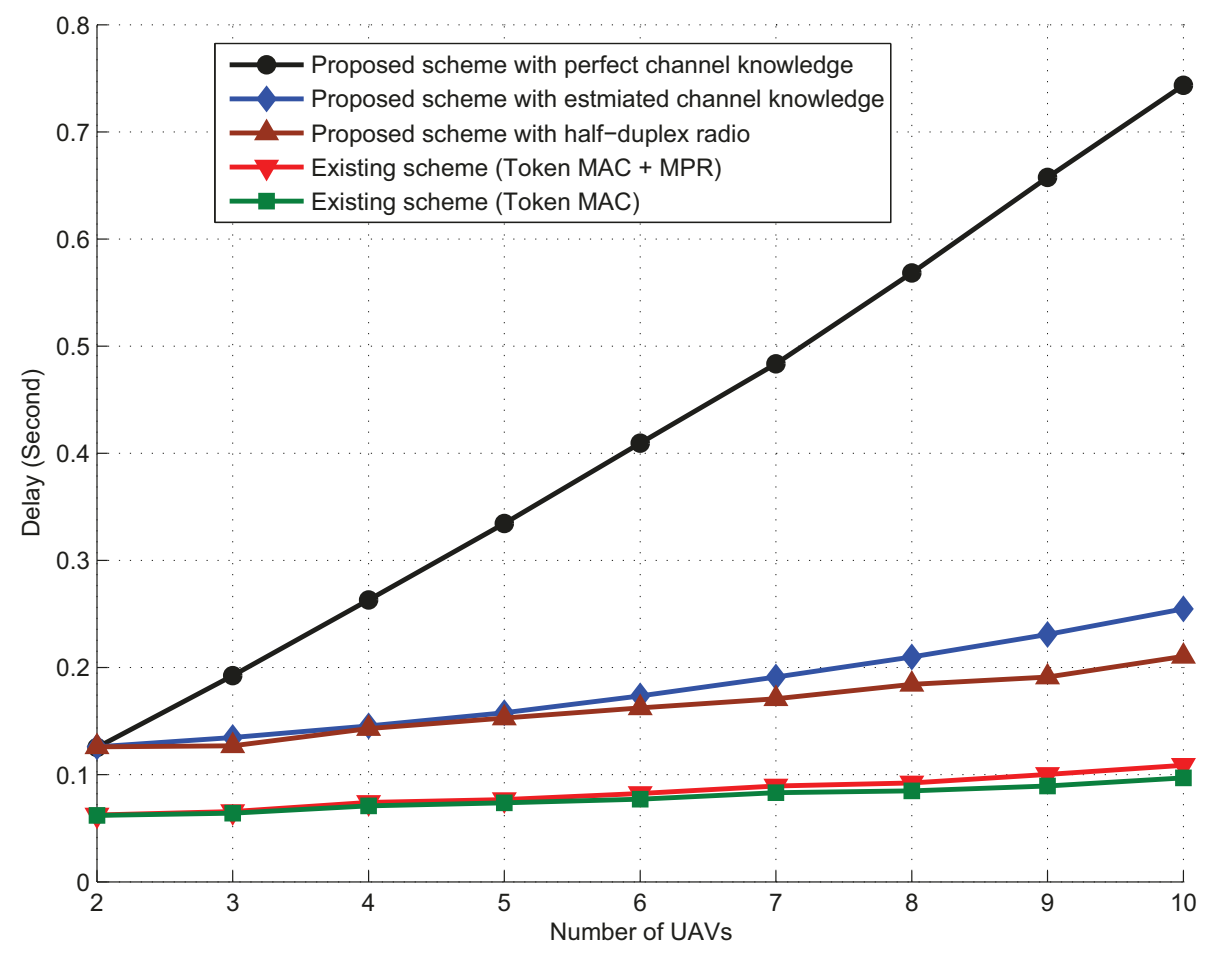

Figure 6.6: Delay performance versus the number of UAVs.

MAC, the transmission chance is only used by the token holder. Hence, as the number of UAVs increases, the delay increases almost linearly while the throughput is nearly constant. The Token + MPR scheme can improve the throughput and delay performance compared to the traditional token ring MAC, since more packets can be transmitted in a given slot via MPR. With channel state information, the optimal decisions are made to maximize the throughput and minimize the delay, therefore, the performance raises even just half-duplex radio is employed. By considering both full-duplex radios and MPR capability, the proposed scheme can further improve the throughput and delay performance. To sum up, with joint optimization over the physical layer with full-duplex radios and MPR capability and MAC layer, we can attain significant performance improvement in UAV ad-hoc networks. Besides, we can see that the performance of the proposed optimization scheme with imperfect channel knowledge is able to approximate the performance with perfect channel 
knowledge. The gap between the two proposed schemes enlarges as the number of UAVs increases. This is because when the number of UAVs increases the searching space is getting larger, hence it needs more iterations for the algorithm to find out the optimal point.

\subsubsection{The Effect of Self-Interference Cancellation Factor}

Recall that the self-interference cancellation factor $\psi$ is a parameter to represent the effect of self-interference cancellation from the transmitting power. To investigate the effect of the imperfect interference cancellation, we conduct simulations with various $\psi$ settings. From Fig. 6.7 and Fig. 6.8, we can see that, with a smaller $\psi$, we can get better performance. But the benefit from that becomes less significant when the value of $\psi$ reaches $10^{-4}$. In particular, both throughput and delay under settings of $\psi=10^{-5}$ and $\psi=10^{-6}$ are very close. This is because, when the self-interference is very small, it has little impact on the system performance.

\subsubsection{The Effect of Channel Estimate Error}

As we discuss in the former sections, a sufficient condition for Algorithm 6 to converge is that the channel estimation error is bounded. In simulations, to investigate the effect of channel estimate error, we define the channel estimate error rate as

$$
\frac{\mathbb{E}[\text { estimated channel gain - actual channel gain }]}{\mathbb{E}[\text { actual channel gain }]} .
$$

The results are shown in Fig. 6.9. We can see that, as the channel estimation error rate increases, the throughput decreases, and the delay increases. This is because the UAV nodes will make more wrong decisions when the channel estimation error rate increases. 


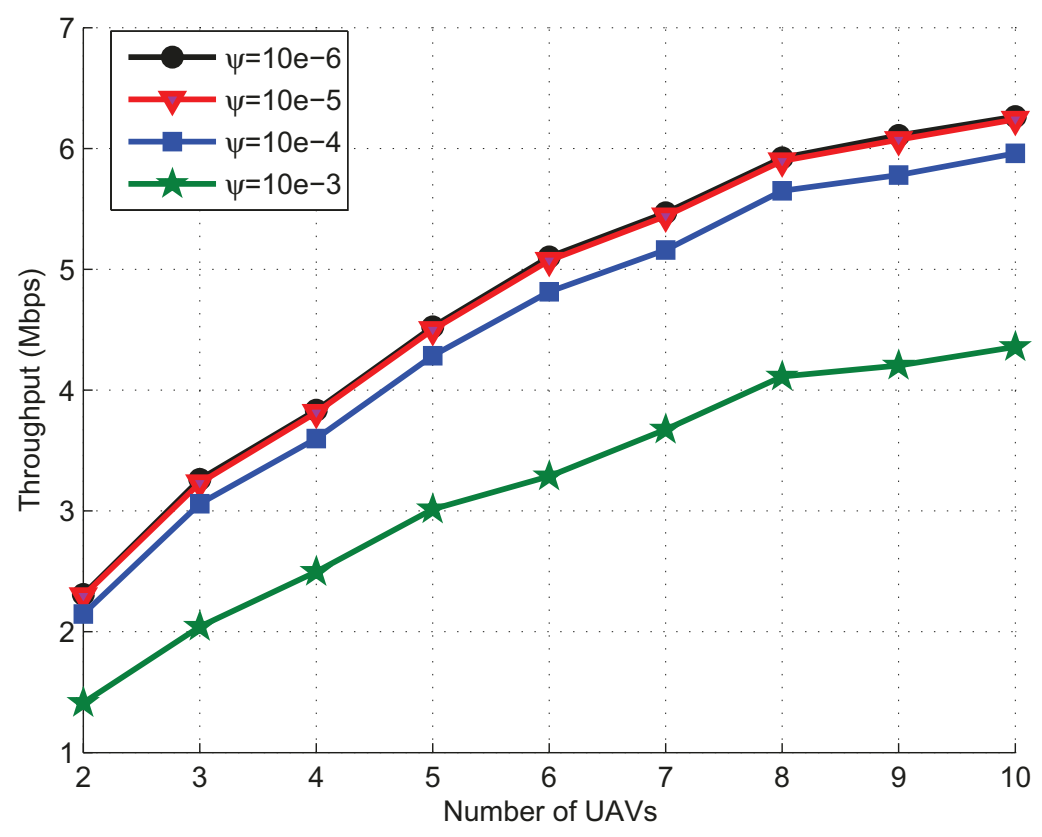

Figure 6.7: The effect of self-interference cancellation factor $\psi$ on throughput.

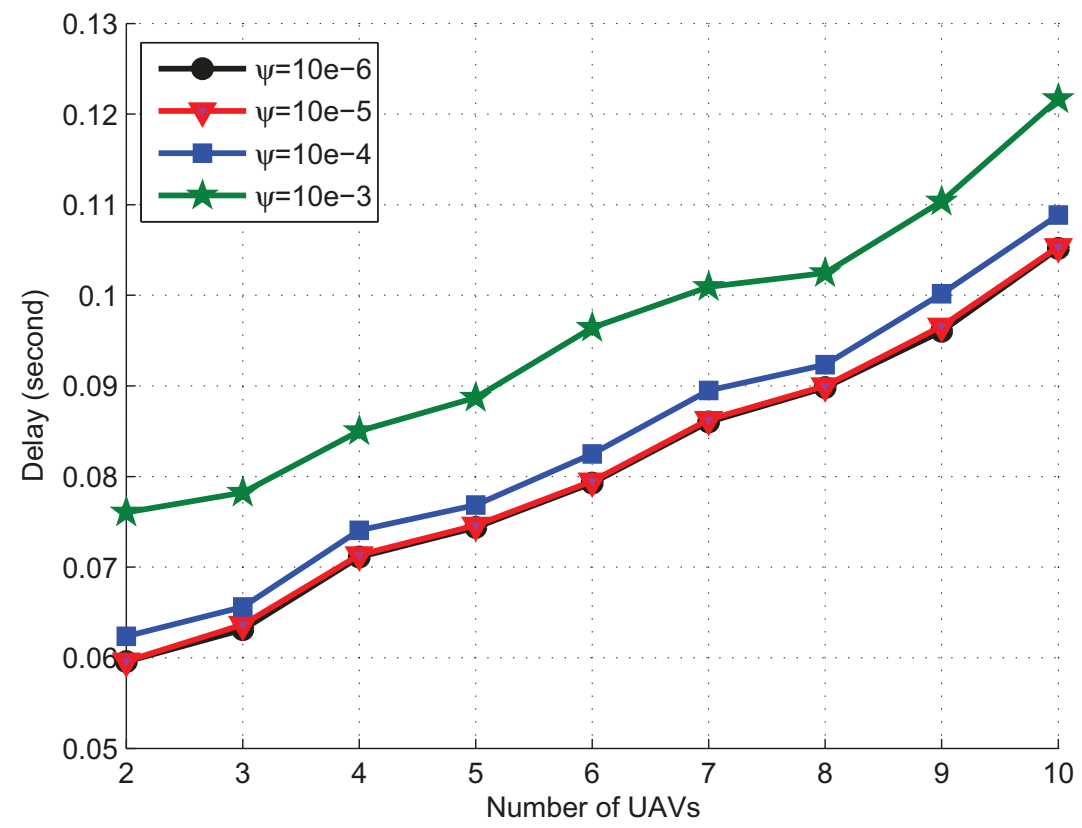

Figure 6.8: The effect of self-interference cancellation factor $\psi$ on delay. 

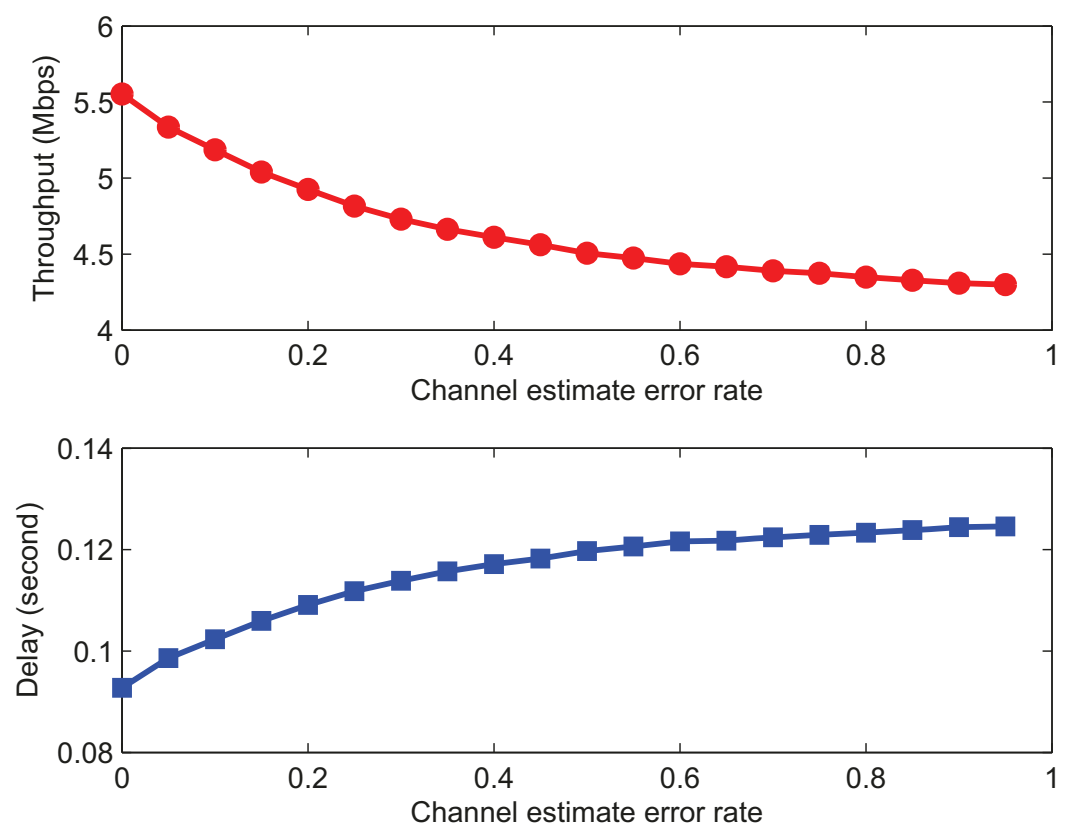

Figure 6.9: The effect of channel estimate error rate.

\subsubsection{Convergence and Tracking the Time-Varying Channels}

First we study the convergence property of Algorithm 6 in the static channel environment and Algorithm 7 in the time-varying channel environment with imperfect channel information. The subfigure at the top of Fig. 6.10 is a single run of Algorith$\mathrm{m} 6$ in a network of 8 nodes. The subfigure at the bottom of Fig. 6.10 is the average over 100 runs in the same network. As we can see, the objective values obtained in the beginning of the simulation are sub-optimal due to the fact that both Algorithm 6 and Algorithm 7 are randomized algorithms. However, after a number of iterations, the objective values converge to an the optimal values thanks to the the attraction property [79] of the two algorithms. If we change the number of nodes in the network, we can observe the similar convergence characteristics. Due to the limited space, the other convergence results in different network settings are omitted.

To evaluate the tracking performance of Algorithm 7, we run simulations with 

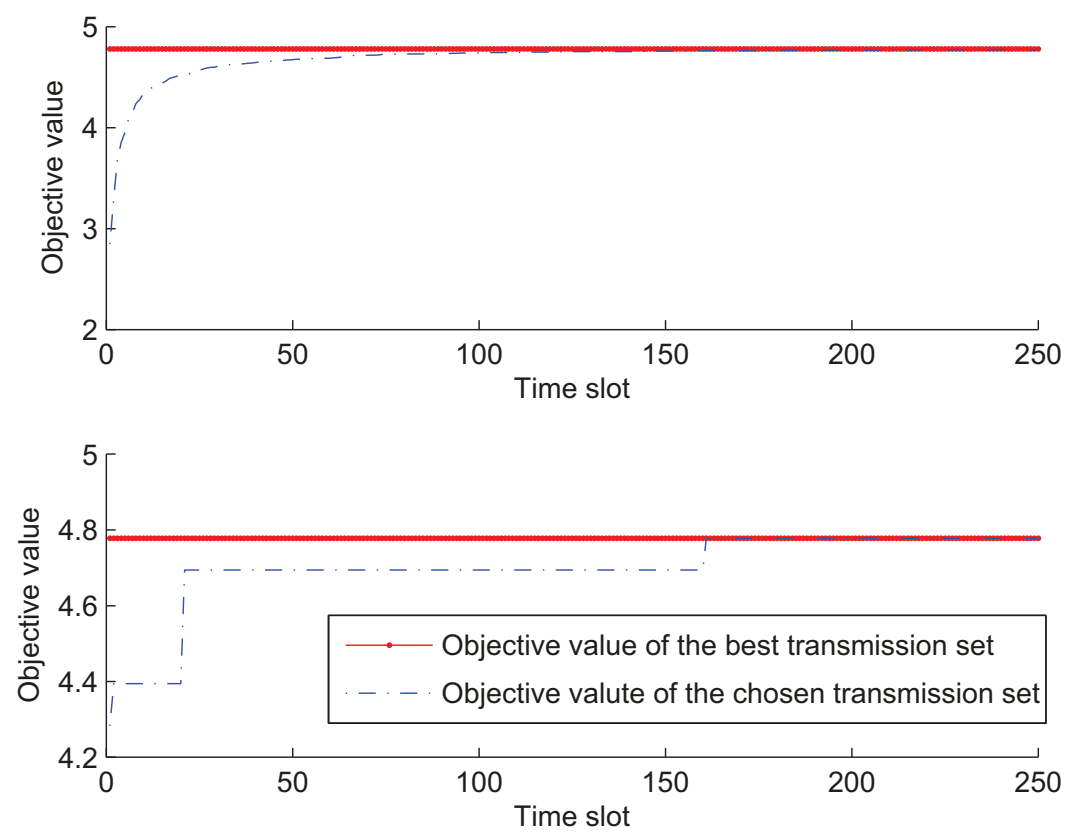

Figure 6.10: Convergence of the objective values in the static channel environment.

time-varying Rayleigh fading channels. From the result shown in Fig. 6.11, we can see the algorithm is able to track the global optimal when the channel is timevarying. Note that the above results are the values of the objective function, which is a combination of the throughput and delay, as defined in (6.12).

\subsubsection{Chapter Summary}

In this chapter, we investigate the effect of recent development of multi-packet reception and full-duplex radio technologies on the media access control schemes. We propose a token-based medium access control scheme for UAV ad-hoc networks to take advantage the advanced physical layer technologies. We consider both system throughput and packet delay, and formulate the MAC scheme in the presence of perfect and imperfect channel state information as a combinatorial optimization problem 


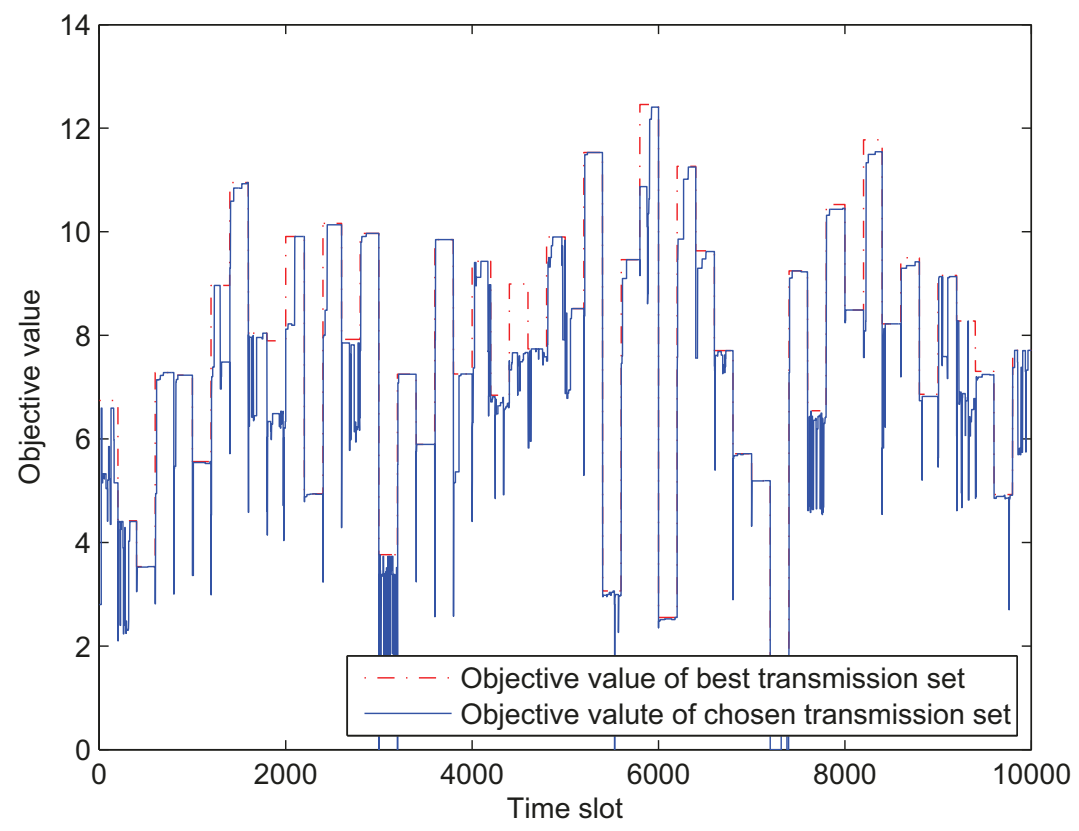

Figure 6.11: Convergence of the objective values in the time-varying channel environment.

and a discrete stochastic optimization problem, respectively. A stochastic approximation algorithm is presented to solve such discrete stochastic optimization problem in time-varying fading channel. We showed that physical layer techniques, such as full-duplex radios and multi-packet reception capability, have significant impact on the system performance of UAV ad-hoc networks. Our simulation results show that the proposed scheme can significantly improve system performance, including the increase of throughput and the decrease of packet delay, compared with the existing schemes. 


\section{Chapter 7}

\section{Conclusions and Future Work}

In this dissertation, we have thoroughly studied the effects of imperfect NSI on four wireless network paradigms including coordinated multi-point cellular networks, cloud radio access networks supporting mobile cloud computing, software-defined device-todevice communications in virtual environments and unmanned aerial vehicle ad hoc networks with multi-packet reception and full-duplex radios. These wireless networks are motivated by the advancement in cloud computing, the necessity to support wireless users to access the cloud, the software-defined networking concept, and the new physical layer technologies. In this chapter, we draw conclusions of the accomplished works and provide some possible directions in the future.

\subsection{Summary}

The proposed schemes in Chapter 3, 4, 5, and 6 address the wireless networking problems given imperfect NSI. In a nutshell, we accomplish the following:

- A decision theoretical approach for uplink CoMP cellular network given delayed CSI was proposed in Chapter 3. We first modeled the wireless channel as a Markov chain. Then the clustering and rate allocation problem was formulated under the framework of networked Markov decision process. Given 
delayed CSI, the network Markov decision process is equivalent to partial observable Markov decision process. An optimal policy was derived to maximize the system throughput. Such policy has the merit of low computation complexity since the majority of the computation can be executed off-line while the on-line phase only involves looking up the table computed from the off-line phase. Simulation results show that the proposed clustering and rate allocation scheme outperforms existing schemes under various settings.

- A dynamic configuration scheme of C-RAN was proposed in Chapter 4. Such scheme aims to provide better support for mobile cloud computing services while addressing the challenge due to outdated CSI. Essentially, the end-to-end performance of TCP flows delivering MCC services is optimized. A TCP-proxy is assumed in the system combined by wireline and wireless networks. The decision theoretic framework maximizes the throughput of MCC services subject to expected user response time which is crucial for many MCC services. We further investigated the trade-off between the systematic efficiency and fairness among MCC users by introducing a parameter to tune the trade-off in the formulation. Computer simulations show that the proposed scheme can achieve significant gains over existing schemes, especially when CSI delay is large and mobility is high.

- A software-defined D2D communication scheme in virtual wireless networks was proposed and the resource sharing problem was tackled in Chapter 5. Our formulation allows a pool of radio resources to be shared by multiple InPs and multiple communications modes. This leads to extremely flexible radio resource utilization by choosing the best communication mode via the best InP. In Chapter 5, the user utility was modeled as a function of the resource sharing decision and the network-wide user welfare is maximized. We assume 
the network application can only have access to noisy NSI; a resource sharing scheme based on discrete stochastic approximation algorithm was developed, accordingly. Extensive simulations show that mobile users can benefit from both wireless network virtualization and software defined D2D communication, and that our proposed scheme can obtain considerable performance gain under practical network settings.

- A media access control scheme for UAV ad hoc networks employing multi-packet reception and full-duplex radio was proposed in Chapter 6. Given perfect CSI, the transmission scheduling problem turns out to be an integer programming; given noisy CSI, it turns out to be a discrete stochastic optimization problem, which is addressed by discrete stochastic approximation algorithms. Such MAC can have better performance than existing schemes both when perfect and imperfect CSI is assumed. By computer simulations, we showed that the use of physical layer full-duplex radios and multi-packet reception capability have significant impacts on the performance of UAV ad-hoc networks. In addition, we showed that throughput and packet delay can be improved substantially in the proposed MAC scheme.

\subsection{Future Work}

A number of interesting research problems rise during the course of the investigations reported in this dissertation.

- The first one is to study the trade-off between complexity and performance in the proposed scheme for CoMP cellular networks with delayed channel state information. The biggest limitation of the Markov decision process formulation is the so-called curse of dimensionality issue, which hinders the state space to be 
large enough to allow more accurate description. In fact, the high dimensionality problem is a general research problem in machine learning community [165]. There are also many proposals to address this problem. As an interesting direction to explore, we can apply state-of-the-art machine learning algorithms in wireless networks. The complexity-performance trade-off is a new topic in schemes employing similar approaches. It is of potential practical benefits because in real life, high computation cost is usually not allowed; instead, the performance is compromised on suboptimal solutions with low computation cost.

- For the schemes presented, we assume that wireless nodes can use either fullpower or zero-power. That means the potential benefit of power control is not fully investigated. Indeed, in wireless networks, the power control problem has been investigated for quite a long time. For instance, in time domain and frequency domain, the optimal power control scheme has a water-filling structure. However, we still do not know whether such structure remains optimal when the dynamics of the network topology is considered. Meanwhile, it is also valuable if a joint optimization framework is developed to have dynamic topology configuration and power control fully explored.

- Another fundamental question lies on the description of NSI. The first related question is the quantization of QSI, a component of NSI. In contrast to CSI quantization for which there have been several proposals, the QSI quantization is a field almost uninvestigated. We still do not know to what extend the different QSI quantization methods will affect system performance. A first step to study this open question might be to apply the various CSI quantization schemes on QSI; but since the inherent nature of CSI and QSI is very different, such approach might not be able to hit the point. The second question is a more 
general question regarding how to define NSI. In this dissertation, NSI is simply considered as CSI plus NSI or merely CSI. However, there might be some other way $(\mathrm{s})$ to characterize the dynamics of wireless networking system, for example, mean values or variance of some parameters. The two questions are correlated since given different quantization method the statistics might change.

- Another direction to explore in the future is to introduce the Lyapunov drift into the formulation when QSI needs to be considered. With Lyapunov drift and optimization, we can stabilize the queuing network and can trade the stability of the system for the performance such as the sum user utilities. However, most of current research assume that the network state information is perfect, in the future, we can try to address the stability issue provided imperfect NSI.

- The last promising direction is to explore the interaction of big data technologies with wireless networks or computer networks in general. One question related to computer communications is how to design the infrastructure to support big data analytic. On the other hand, the visualization and prediction technologies developed by data scientists can help in the design and development of next generation networks in terms of architectures, principles, etc. 


\section{List of References}

[1] S. Adlakha, S. Lall, and A. Goldsmith, "Information state for Markov decision processes with network delays," in Proc. of IEEE CDC'08, pp. 3840 -3847, Dec. 2008.

[2] E. Pateromichelakis, M. Shariat, A. u. Quddus, and R. Tafazolli, "On the evolution of multi-cell scheduling in 3GPP LTE / LTE-A," IEEE Commun. Surveys $\&$ Tutorials, pp. 1-17, 2012.

[3] R. Irmer, H. Droste, P. Marsch, M. Grieger, G. Fettweis, S. Brueck, H.-P. Mayer, L. Thiele, and V. Jungnickel, "Coordinated multipoint: concepts, performance, and field trial results," IEEE Comm. Mag., vol. 49, no. 2, pp. 102-111, february 2011.

[4] K. M. S. Huq, S. Mumtaz, F. B. Saghezchi, J. Rodriguez, and R. L. Aguiar, "Energy efficiency of downlink packet scheduling in CoMP," Transactions on Emerging Telecommunications Technologies, 2013.

[5] W. Choi and J. Andrews, "Downlink performance and capacity of distributed antenna systems in a multicell environment," IEEE Trans. Wireless Commun., vol. 6, no. 1, pp. 69-73, Jan. 2007.

[6] S. Hanly, "Capacity and power control in spread spectrum macrodiversity radio networks," IEEE Trans. Commun., vol. 44, no. 2, pp. 247-256, Feb 1996.

[7] D. Gesbert, S. Hanly, H. Huang, S. Shamai Shitz, O. Simeone, and W. Yu, "Multi-cell MIMO cooperative networks: a new look at interference," IEEE J. Sel. Areas Commun., vol. 28, no. 9, pp. 1380-1408, december 2010.

[8] A. Papadogiannis, D. Gesbert, and E. Hardouin, "A dynamic clustering approach in wireless networks with multi-cell cooperative processing," in IEEE ICC'08, pp. 4033 -4037, May 2008. 
[9] S. Zhou, J. Gong, Z. Niu, Y. Jia, and P. Yang, "A decentralized framework for dynamic downlink base station cooperation," in Proc. of IEEE GLOBECOM'09, pp. 1-6, IEEE, Dec. 2009.

[10] J. Liu and D. Wang, "An improved dynamic clustering algorithm for multi-user distributed antenna system," in Wireless Communications $\& 5$ Signal Processing, 2009. WCSP 2009. International Conference on, 2009.

[11] J.-M. Moon and D.-H. Cho, "Efficient cell-clustering algorithm for inter-cluster interference mitigation in network MIMO systems," IEEE Commun. Let., vol. 15, no. 3, pp. 326-328, Mar. 2011.

[12] Y. Cui, Q. Huang, and V. Lau, "Queue-aware dynamic clustering and power allocation for network MIMO systems via distributed stochastic learning," IEEE Trans. Signal Proc., vol. 59, no. 3, pp. 1229 -1238, Mar. 2011.

[13] W. Yu, T. Kwon, and C. Shin, "Multicell coordination via joint scheduling, beamforming and power spectrum adaptation," in Proc. IEEE INFOCOM 2011, pp. 2570-2578, 2011.

[14] H. Dahrouj and W. Yu, "Coordinated beamforming for the multicell multiantenna wireless system," IEEE Trans. Wireless Commun., vol. 9, pp. 17481759, May 2010.

[15] D. J. Love, R. W. Heath, V. K. Lau, D. Gesbert, B. D. Rao, and M. Andrews, "An overview of limited feedback in wireless communication systems," IEEE J. Sel. Areas Commun., vol. 26, no. 8, pp. 1341-1365, Oct. 2008.

[16] R. Bhagavatula and R. W. Heath, "Adaptive bit partitioning for multicell intercell interference nulling with delayed limited feedback," IEEE Trans. Signal Proc., vol. 59, no. 8, pp. 3824-3836, Aug. 2011.

[17] P. Marsch and G. Fettweis, "Uplink CoMP under a constrained backhaul and imperfect channel knowledge," IEEE Trans. Wireless Commun., vol. 10, no. 6, pp. 1730-1742, Jun 2011.

[18] F. Diehm and G. Fettweis, "On the impact of signaling delays on the performance of centralized scheduling for joint detection cooperative cellular systems," in Proc. IEEE Wireless Communications and Networking Conference(WCNC), 2011, pp. 1897-1902, march 2011. 
[19] F. Diehm and G. Fettweis, "Centralized scheduling for joint decoding cooperative networks subject to signalling delays," in 2011 IEEE Vehicular Technology Conference (VTC Fall),, pp. 1-5, Sept.

[20] K. Huang, B. Mondal, R. Heath, and J. Andrews, "Effect of feedback delay on multi-antenna limited feedback for temporally-correlated channels," in Proc. IEEE GLOBECOM'06., pp. 105-109, dec. 2006.

[21] K. Huang, B. Mondal, R. Heath, and J. Andrews, "Multi-antenna limited feedback for temporally-correlated channels: Feedback compression," in Proc. IEEE GLOBECOM'06., pp. 326 -330, dec. 2006.

[22] A. Duel-Hallen, "Fading channel prediction for mobile radio adaptive transmission systems," Proc. IEEE, vol. 95, no. 12, pp. 2299-2313, Dec. 2007.

[23] P. Marsch, Coordinated Multi-Point under a constrained backhaul and imperfect channel knowledge. PhD thesis, TU Dresden, Jun. 2010.

[24] J. Alcaraz, F. Cerdan, and J. Garcia-Haro, "Optimizing TCP and RLC interaction in the UMTS radio access network," IEEE Network, vol. 20, no. 2, pp. 56 -64, Apr. 2006.

[25] H. Vu and S. Hanly, "A study of TCP performance and buffer occupancy over a fading wireless link," in IEEE Global Telecommunications Conference, 2001. GLOBECOM '01, vol. 6, pp. 3478-3482 vol.6, 2001.

[26] A. Toledo, X. Wang, and B. Lu, "A cross-layer TCP modelling framework for MIMO wireless systems," IEEE Trans. Wireless Commun., vol. 5, no. 4, pp. 920-929, Apr. 2006.

[27] J.P. Singh, Y. Li, N. Bambos, A. Bahai, B. Xu, and G. Zimmermann, "TCP performance dynamics and link-layer adaptation based optimization methods for wireless networks," IEEE Trans. Wireless Commun., vol. 6, no. 5, pp. 1864 -1879 , May 2007.

[28] M. Assaad and D. Zeghlache, "Cross-layer design in HSDPA system to reduce the TCP effect," IEEE J. Sel. Areas Commun., vol. 24, no. 3, no. 3, pp. 614-625, 2006.

[29] M. Ghaderi, A. Sridharan, H. Zang, D. Towsley, and R. Cruz, "TCP-Aware channel allocation in CDMA networks," IEEE Trans. Mobile Comput., vol. 8, no. 1, pp. $14-28$, Jan. 2009. 
[30] Majid Ghaderi Dehkordi, A. Sridharan, and H. Zang, "TCP-aware resource allocation in wireless networks," Jan. 2011. U.S. Classification: 370/468.

[31] C. Luo, F. R. Yu, H. Ji, and V. C. Leung, "Cross-layer design for TCP performance improvement in cognitive radio networks," IEEE Trans. Veh. Tech., vol. 59, no. 5, pp. 2485-2495, Jun. 2010.

[32] O. Habachi, H.-P. Shiang, M. van der Schaar, and Y. Hayel, "Online learning based congestion control for adaptive multimedia transmission," IEEE Trans. Signal Proc., vol. 61, no. 6, pp. 1460-1469, Mar. 2013.

[33] A. Abouzeid, S. Roy, and M. Azizoglu, "Comprehensive performance analysis of a TCP session over a wireless fading link with queueing," IEEE Trans. Wireless Commun., vol. 2, no. 2, no. 2, pp. 344-356, 2003.

[34] W. Wei, C. Zhang, H. Zang, J. Kurose, and D. Towsley, "Inference and evaluation of split-connection approaches in cellular data networks," in Proc. Passive and Active Measurement Conference, 2006.

[35] A. Pathak, Y. A. Wang, C. Huang, A. Greenberg, Y. C. Hu, R. Kern, J. Li, and K. W. Ross, "Measuring and evaluating TCP splitting for cloud services," in Proc. 11th Int'l Conf. Passive and Active Measurement (PAM'10), (Berlin, Heidelberg), pp. 41-50, Springer-Verlag, 2010.

[36] F. Baccelli, G. Carofiglio, and S. Foss, "Proxy caching in split TCP: dynamics, stability and tail asymptotics," in IEEE INFOCOM 2008. The 27th Conference on Computer Communications, pp. 131-135, 2008.

[37] M. Tariq, A. Zeitoun, V. Valancius, N. Feamster, and M. Ammar, "Answering what-if deployment and configuration questions with wise," in Proceedings of the ACM SIGCOMM 2008 conference on Data communication, SIGCOMM '08, (New York, NY, USA), pp. 99-110, ACM, 2008.

[38] Y. Chen, S. Jain, V. K. Adhikari, and Z.-L. Zhang, "Characterizing roles of front-end servers in end-to-end performance of dynamic content distribution," in Proc. ACM SIGCOMM Internet Measurement Conf. (IMC'11), (New York, NY, USA), pp. 559-568, 2011.

[39] N. McKeown, T. Anderson, H. Balakrishnan, G. Parulkar, L. Peterson, J. Rexford, S. Shenker, and J. Turner, "OpenFlow: enabling innovation in campus networks," SIGCOMM Comput. Commun. Rev., vol. 38, no. 2, Mar. 2008. 
[40] R. Jain and S. Paul, "Network virtualization and software defined networking for cloud computing: a survey," IEEE Comm. Mag., vol. 51, no. 11, no. 11, pp. 24-31, 2013.

[41] X. Costa-Perez, J. Swetina, T. Guo, R. Mahindra, and S. Rangarajan, "Radio access network virtualization for future mobile carrier networks," IEEE Comm. Mag., vol. 51, no. 7, no. 7, pp. 27-35, 2013.

[42] A. Gudipati, D. Perry, L. E. Li, and S. Katti, "SoftRAN: Software defined radio access network," in Proc. ACM HotSDN'13, 2013.

[43] L. E. Li, Z. M. Mao, and J. Rexford, "Cellsdn: Software-defined cellular networks," tech. rep., Technical report, Princeton University Computer Science Technical Report, 2012.

[44] M. Bansal, J. Mehlman, S. Katti, and P. Levis, "OpenRadio: a programmable wireless dataplane," in Proc. of the first workshop on Hot topics in software defined networks, pp. 109-114, ACM, 2012.

[45] X. Jin, L. E. Li, L. Vanbever, and J. Rexford, "SoftCell: taking control of cellular core networks," arXiv e-print 1305.3568, May 2013.

[46] K. Pentikousis, Y. Wang, and W. Hu, "Mobileflow: Toward software-defined mobile networks," IEEE Comm. Mag., vol. 51, no. 7, no. 7, pp. 44-53, 2013.

[47] M. Chiosi and et al, "Network functions virtualisation introductory white paper i," white paper, AT\&T and et al, Oct. 2012.

[48] H. Wen, P. K. Tiwary, and T. Le-Ngoc, "Wireless virtualization," in Wireless Virtualization, pp. 41-81, Springer, 2013.

[49] R. Kokku, R. Mahindra, H. Zhang, and S. Rangarajan, "NVS: a substrate for virtualizing wireless resources in cellular networks," IEEE/ACM Trans. Netw., vol. 20, no. 5, pp. 1333-1346, Oct. 2012.

[50] C. Liang and F. R. Yu, "Wireless network virtualization: A survey, some research issues and challenges," IEEE Commun. Surveys 8 Tutorials, 2014. to appear.

[51] A. Asadi, Q. Wang, and V. Mancuso, "A survey on device-to-device communication in cellular networks," CoRR, vol. abs/1310.0720, 2013. 
[52] N. K. Pratas and P. Popovski, "Low-rate machine-type communication via wireless device-to-device (D2D) links," CoRR, vol. abs/1305.6783, 2013.

[53] K. Doppler, M. Rinne, C. Wijting, C. Ribeiro, and K. Hugl, "Device-to-device communication as an underlay to LTE-advanced networks," IEEE Comm. Mag., vol. 47, no. 12, no. 12, pp. 42-49, 2009.

[54] M. J. Yang, S. Y. Lim, H. J. Park, and N. H. Park, "Solving the data overload: Device-to-device bearer control architecture for cellular data offloading," IEEE Veh. Tech. Mag., vol. 8, no. 1, no. 1, pp. 31-39, 2013.

[55] G. Fodor, E. Dahlman, G. Mildh, S. Parkvall, N. Reider, G. Miklos, and Z. Turanyi, "Design aspects of network assisted device-to-device communications," IEEE Comm. Mag., vol. 50, no. 3, no. 3, pp. 170-177, 2012.

[56] N. Reider and G. Fodor, "A distributed power control and mode selection algorithm for D2D communications," EURASIP Journal on Wireless Communications and Networking, vol. 2012, no. 1, pp. 1-25, Dec. 2012.

[57] Y.-D. Lin and Y.-C. Hsu, "Multihop cellular: a new architecture for wireless communications," in pINF 2000, vol. 3, pp. 1273-1282 vol.3, 2000.

[58] X. Lin, J. G. Andrews, and A. Ghosh, "A comprehensive framework for device-to-device communications in cellular networks," arXiv preprint arXiv:1305.4219, 2013.

[59] K. Doppler, C.-H. Yu, C. Ribeiro, and P. Janis, "Mode selection for device-todevice communication underlaying an LTE-Advanced network," in Proc. IEEE WCNC'10, pp. 1-6, 2010.

[60] C.-H. Yu, K. Doppler, C. Ribeiro, and O. Tirkkonen, "Resource sharing optimization for device-to-device communication underlaying cellular networks," IEEE Trans. Wireless Commun., vol. 10, no. 8, no. 8, pp. 2752-2763, 2011.

[61] P. Phunchongharn, E. Hossain, and D. I. Kim, "Resource allocation for deviceto-device communications underlaying lte-advanced networks," IEEE Wireless Comm., vol. 20, no. 4, no. 4, 2013.

[62] W.-T. Chen, J.-C. Liu, T.-K. Huang, and Y.-C. Chang, "TAMMAC: An adaptive multi-channel MAC protocol for MANETs," IEEE Trans. Wireless Commun., vol. 7, no. 11, pp. $4541-4545$, Nov. 2008. 
[63] T. D. Ho, J. Park, and S. Shimamoto, "QoS constraint with prioritized frame selection CDMA MAC protocol for WSN employing UAV," in Proc. IEEE Globecom'10 Workshop on Wireless Netw. for UAVs, pp. 1826-1830, Dec. 2010.

[64] M. Ergen, D. Lee, R. Sengupta, and P. Varaiya, "WTRP - wireless token ring protocol," IEEE Trans. Veh. Tech., vol. 53, no. 6, pp. 1863-1881, Nov. 2004.

[65] I.-S. Liu, F. Takawira, and H.-J. Xu, "A hybrid token-CDMA MAC protocol for wireless ad hoc networks," IEEE Trans. Mobile Comput., vol. 7, no. 5, pp. 557-569, May 2008.

[66] N. Malpani, Y. Chen, N. Vaidya, and J. Welch, "Distributed token circulation in mobile ad hoc networks," IEEE Trans. Mobile Comput., vol. 4, no. 2, pp. 154165, Mar. 2005.

[67] D. L. Gu, H. Ly, X. Hong, M. Gerla, G. Pei, and Y.-Z. Lee, "C-ICAMA, a centralized intelligent channel assigned multiple access for multi-layer ad-hoc wireless networks with UAVs," in Proc. IEEE WCNC'00, pp. 879-884, 2000.

[68] C.-K. Lin, H. T. Kung, T.-H. Lin, S. J. Tarsa, and D. Vlah, "Achieving high throughput ground-to-UAV transport via parallel links," in Proc. ICCCN'11, (Maui, Hawaii), Aug. 2011.

[69] J. Zhou and X. Yuan, "A study of dynamic routing and wavelength assignment with imprecise network state information," in Parallel Processing Workshops, 2002. Proceedings. International Conference on, pp. 207-213, IEEE, 2002.

[70] R. A. Guérin and A. Orda, "Qos routing in networks with inaccurate information: Theory and algorithms," IEEE/ACM Trans. Netw., vol. 7, no. 3, pp. 350364, Jun. 1999.

[71] L. Ying and S. Shakkottai, "On throughput optimality with delayed networkstate information," IEEE Trans. Inform. Theory, vol. 57, no. 8, no. 8, pp. 51165132, 2011.

[72] M. Puterman, Markov Decision Processes: Discrete Stochastic Dynamic Programming. John Wiley \& Sons, Inc., 1994.

[73] S. Adlakha, S. Lall, and A. Goldsmith, "Networked Markov decision processes with delays," IEEE Trans. Auto. Control, vol. 57, no. 4, pp. 1013-1018, Apr. 2012. 
[74] L. P. Kaelbling, M. L. Littman, and A. R. Cassandra, "Planning and acting in partially observable stochastic domains," Artificial Intelligence, vol. 101, no. 12, pp. 99-134, May 1998.

[75] S. Andradottir, "A global search method for discrete stochastic optimization," SIAM J. Opt., vol. 6, no. 2, no. 2, pp. 513-530, 1996.

[76] J. R. Swisher, P. D. Hyden, S. H. Jacobson, and L. W. Schruben, "A survey of simulation optimization techniques and procedures," in Proc. of the 2000 Winter Simulation Conference, vol. 1, pp. 119-128, IEEE, 2000.

[77] D. Goldsman and B. L. Nelson, "Ranking, selection and multiple comparisons in computer simulation," in Proc. of the 1994 Winter Simulation Conference, pp. 192-199, IEEE, 1994.

[78] J. Hsu, Multiple Comparisons: Theory and Methods. CRC Press, 1996.

[79] I. Berenguer, X. Wang, and V. Krishnamurthy, "Adaptive MIMO antenna selection via discrete stochastic optimization," IEEE Trans. Signal Proc., vol. 53, no. 11, pp. 4315-4329, nov. 2005.

[80] Z. Zheng, A. A. Dowhuszko, and J. Hmlinen, "Interference management for LTE-Advanced Het-Nets: stochastic scheduling approach in frequency domain," Transactions on Emerging Telecommunications Technologies, vol. 24, no. 1, no. 1, pp. 4-17, 2013.

[81] A. Cimmino, T. Pecorella, R. Fantacci, F. Granelli, T. F. Rahman, C. Sacchi, C. Carlini, and P. Harsh, "The role of small cell technology in future smart city applications," Transactions on Emerging Telecommunications Technologies, vol. 25, no. 1, no. 1, pp. 11-20, 2014.

[82] A. Ghosh, N. Mangalvedhe, R. Ratasuk, B. Mondal, M. Cudak, E. Visotsky, T. Thomas, J. Andrews, P. Xia, H. Jo, H. Dhillon, and T. Novlan, "Heterogeneous cellular networks: From theory to practice," IEEE Comm. Mag., vol. 50, no. 6, pp. $54-64$, Jun. 2012.

[83] 3rd Generation Partnership Project, "Reply LS to R3-070527/R1-071242 on Backhaul (X2 interface) Delay," tech. rep., 3rd Generation Partnership Project, 2007.

[84] L. Scalia, T. Biermann, C. Choi, K. Kozu, and W. Kellerer, "Power-Efficient Mobile Backhaul Design for CoMP Support in Future Wireless Access Systems," 
in Proc. IEEE INFOCOM'11 Workshop on Green Comm. Networking, Apr. 2011.

[85] A. Goldsmith, M. Effros, R. Koetter, M. Medard, A. Ozdaglar, and L. Zheng, "Beyond Shannon: the quest for fundamental performance limits of wireless ad hoc networks," IEEE Comm. Mag., vol. 49, no. 5, pp. 195 -205, May 2011.

[86] T. Coleman, "A stochastic control viewpoint on posterior-matching-style feedback communication schemes," in Proc. IEEE Int'l Symp. Information Theory, July 2009.

[87] P. Sadeghi, R. Kennedy, P. Rapajic, and R. Shams, "Finite-state Markov modeling of fading channels - a survey of principles and applications," IEEE Signal Processing Magazine, vol. 25, no. 5, pp. 57-80, Sep. 2008.

[88] F. Babich, G. Lombardi, and E. Valentinuzzi, "Variable order Markov modeling for LEO mobile satellite channels," Electron. Lett., vol. 35, no. 8, pp. 621-623, Apr. 1999.

[89] F. Babich and G. Lombardi, "A measurement based Markov model for the indoor propagation channel," in Proc. IEEE VTC'97, vol. 1, (Phoenix, AZ), pp. 77-81, May 1997.

[90] H. S. Wang and N. Moayeri, "Finite-state Markov channel - a useful model for radio communication channels," IEEE Trans. Veh. Tech., vol. 44, no. 1, pp. 163 -171, Feb. 1995.

[91] C. Pimentel, T. Falk, and L. Lisboa, "Finite-state Markov modeling of correlated Rician-fading channels," IEEE Trans. Veh. Tech., vol. 53, no. 5, pp. 1491 - 1501, Sept. 2004.

[92] C. Iskander and P. Mathiopoulos, "Fast simulation of diversity Nakagami fading channels using finite-state Markov models," IEEE Trans. Broadcasting, vol. 49, no. 3, pp. 269 - 277, Sept. 2003.

[93] D. Tse and P. Viswanath, Fundamentals of Wireless Communication. Cambridge Univ Press, 2005.

[94] Q. Zhang and S. Kassam, "Finite-state Markov model for Rayleigh fading channels," IEEE Trans. Commun., vol. 47, no. 11, pp. 1688 -1692, Nov. 1999. 
[95] J. M. Park and G. Hwang, "Mathematical modeling of Rayleigh fading channels based on finite state Markov chains," IEEE Commun. Let., vol. 13, no. 10, pp. $764-766$, Oct. 2009.

[96] S. Lloyd, "Least squares quantization in PCM," IEEE Trans. Inform. Theory, vol. 28, no. 2, no. 2, pp. 129-137, 1982.

[97] M. Chu, D. Goeckel, and W. Stark, "On the design of Markov models for fading channels," in Vehicular Technology Conference, 1999. VTC 1999 - Fall. IEEE VTS 50th, vol. 4, pp. 2372-2376 vol.4, 1999.

[98] H.-S. Wang and N. Moayeri, "Modeling, capacity, and joint source/channel coding for Rayleigh fading channels," in 43rd IEEE Vehicular Technology Conference, 1993, pp. 473-479, 1993.

[99] S. Pissanetzky, Sparse matrix technology. Academic Press, 1984.

[100] H. Kobayashi, B. L. Mark, and W. Turin, Probability, random processes, and statistical analysis: applications to communications, signal Processing, queueing theory and mathematical finance. Cambridge University Press, Feb. 2012.

[101] J. S. Rosenthal, "Convergence rates for Markov chains," SIAM Review, vol. 37, no. 3, pp. 387-405, Sep. 1995.

[102] L. Rabiner, "A tutorial on hidden Markov models and selected applications in speech recognition," Proc. IEEE, vol. 77, no. 2, pp. 257 -286, Feb. 1989.

[103] C.-X. Wang, X. Hong, H. Wu, and W. Xu, "Spatial-temporal correlation properties of the $3 \mathrm{GPP}$ spatial channel model and the kronecker MIMO channel model," EURASIP J. on Wireless Commun. \& Netw., vol. 2007, 2007.

[104] A. G. Marques, L. M. Lopez-Ramos, G. B. Giannakis, J. Ramos, and A. J. Caamano, "Optimal cross-layer resource allocation in cellular networks using channel- and queue-state information," IEEE Trans. Veh. Tech., vol. 61, no. 6, pp. $2789-2807$, July 2012.

[105] G. Pallis, "Cloud computing: the new frontier of internet computing," IEEE Internet Computing, vol. 14, no. 5, no. 5, pp. 70-73, 2010.

[106] NIST, "The NIST definition of cloud computing (draft)," Tech. Rep. Special Publication 800-145 (Draft), Jan. 2011. 
[107] H. T. Dinh, C. Lee, D. Niyato, and P. Wang, "A survey of mobile cloud computing: architecture, applications, and approaches," Wireless Communications and Mobile Computing, 2011.

[108] S. Wang and S. Dey, "Adaptive mobile cloud computing to enable rich mobile multimedia applications," IEEE Trans. Multimedia, vol. 15, no. 4, no. 4, pp. 870-883, 2013.

[109] S. Bhaumik, S. P. Chandrabose, M. K. Jataprolu, G. Kumar, A. Muralidhar, P. Polakos, V. Srinivasan, and T. Woo, "CloudIQ: A framework for processing base stations in a data center," in Proc. ACM Mobicom'12, (Istanbul, Turkey), 2012.

[110] China Mobile Research Institute, "C-RAN: the road towards green RAN," tech. rep. http://labs.chinamobile.com/, accessed: 2013-07-18.

[111] O. S.-H. Park, and Simeone, O. Sahin, and S. Shamai, "Robust and efficient distributed compression for cloud radio access networks," IEEE Trans. Veh. Tech., vol. 62, no. 2, no. 2, pp. 692-703, Feb. 2013.

[112] H. Balakrishnan, V. N. Padmanabhan, S. Seshan, and R. H. Katz, "A comparison of mechanisms for improving TCP performance over wireless links," IEEE/ACM Trans. Netw., vol. 5, no. 6, pp. 756-769, Dec. 1997.

[113] N. H. Tran, C. S. Hong, and S. Lee, "Cross-layer design of congestion control and power control in fast-fading wireless networks," IEEE Trans. Parallel and Dist. Systems, vol. 24, no. 2, pp. 260-274, Feb. 2013.

[114] G. Wang and T. S. E. Ng, "The impact of virtualization on network performance of Amazon EC2 data center," in Proc. IEEE INFOCOM'10, (San Diego, CA), Mar. 2010.

[115] M. Satyanarayanan, P. Bahl, R. Caceres, and N. Davies, "The case for VMbased cloudlets in mobile computing," IEEE Pervasive Computing, vol. 8, no. 4, no. 4, pp. 14-23, 2009.

[116] Y. Lin, L. Shao, Z. Zhu, Q. Wang, and R. K. Sabhikhi, "Wireless network cloud: architecture and system requirements," IBM Journal of Research and Development, vol. 54, no. 1, no. 1, pp. 4:1-4:12, 2010.

[117] D. Bojic and N. Europe, "Advanced wireless and optical technologies for smallcell mobile backhaul with dynamic software-defined management," IEEE Comm. Mag., vol. 51, no. 9, no. 9, pp. 86-93, 2013. 
[118] Z. Zhu, P. Gupta, Q. Wang, S. Kalyanaraman, Y. Lin, H. Franke, and S. Sarangi, "Virtual base station pool: Towards a wireless network cloud for radio access networks," in Proc. 8th ACM Int'l Conf. Computing Frontiers, (New York, NY, USA), 2011.

[119] V. Farkas, B. Hder, and S. Novczki, "A split connection tcp proxy in LTE networks," in Information and Communication Technologies (R. Szab and A. Vidcs, eds.), vol. 7479 of Lecture Notes in Computer Science, pp. 263-274, Springer Berlin Heidelberg, 2012.

[120] R. Xie, F. R. Yu, H. Ji, and Y. Li, "Energy-efficient resource allocation for heterogeneous cognitive radio networks with femtocells," IEEE Trans. Wireless Commun., vol. 11, no. 11, pp. 3910 -3920, Nov. 2012.

[121] A. Gjendemsj, D. Gesbert, G. E. Oien, and S. G. Kiani, "Binary power control for sum rate maximization over multiple interfering links," IEEE Trans. Wireless Commun., vol. 7, no. 8, no. 8, pp. 3164-3173, 2008.

[122] M. Assaad and D. Zeghlache, "Comparison between MIMO techniques in UMTS-HSDPA system," in Proc. IEEE 8th Int'l Sym. Spread Spectrum Techniques and Applications, pp. 874-878, 2004.

[123] J. Padhye, V. Firoiu, D. F. Towsley, and J. F. Kurose, "Modeling TCP Reno performance: a simple model and its empirical validation," IEEE/ACM Trans. Netw., vol. 8, no. 2, pp. 133-145, Apr. 2000.

[124] A. H. Land and A. G. Doig, "An automatic method of solving discrete programming problems," Econometrica, vol. 28, no. 3, pp. 497-520, Jul. 1960.

[125] M. Mitchell, An introduction to Genetic Algorithms. A Bradford Book, Third ed., Feb. 1998.

[126] M. Sipser, Introduction to the Theory of Computation. Cengage Learning, 2006.

[127] A. R. Cassandra, Exact and approximate algorithms for partially observable Markov decision processes. Brown University, 1998.

[128] R. Jain, D.-M. Chiu, and W. R. Hawe, A quantitative measure of fairness and discrimination for resource allocation in shared computer system. Eastern Research Lab., DEC, 1984. 
[129] A. Bin Sediq, R. H. Gohary, R. Schoenen, and H. Yanikomeroglu, "Optimal tradeoff between sum-rate efficiency and Jain's fairness index in resource allocation," IEEE Trans. Wireless Commun., vol. 12, no. 7, no. 7, pp. 3496-3509, 2013.

[130] "ns network simulator-version 2," URL: http://www. isi. edu/nsnam/ns.

[131] Y. Cai, F. R. Yu, and G. Senarath, "Optimal clustering and rate allocation for uplink coordinated multi-point (CoMP) systems with delayed channel state information (CSI)," in Proc. IEEE ICC'13, (Budapest, Hungary), June 2013.

[132] K. Huang, MIMO networking with imperfect channel state information. Ph.D. Thesis, The University of Texas at Austin, Texas, USA, 2007.

[133] S. Bu, F. R. Yu, and H. Yanikomeroglu, "Interference-aware energy-efficient resource allocation for heterogeneous networks with incomplete channel state information," IEEE Trans. Veh. Tech., 2014. to appear.

[134] F. Fu and U. Kozat, "Stochastic game for wireless network virtualization," IEEE/ACM Trans. Netw., vol. 21, no. 1, no. 1, pp. 84-97, 2013.

[135] R. Knopp and P. A. Humblet, "Information capacity and power control in single-cell multiuser communications," in IEEE ICC'95, vol. 1, pp. 331-335, IEEE, 1995.

[136] R. Knopp and P. A. Humblet, "Multiple-accessing over frequency-selective fading channels," in IEEE PIMRC'95, vol. 3, p. 1326, IEEE, 1995.

[137] T. Weber, A. Sklavos, and M. Meurer, "Imperfect channel-state information in MIMO transmission," IEEE Trans. Commun., vol. 54, no. 3, pp. 543-552, Mar. 2006.

[138] G. C. Pflug, Optimization of stochastic models: The interface between simulation and optimization. Kluwer Academic Boston, 1996.

[139] H. J. Kushner and G. Yin, Stochastic approximation and recursive algorithms and applications, vol. 35. Springer, 2003.

[140] V. Krishnamurthy, X. Wang, and G. Yin, "Spreading code optimization and adaptation in CDMA via discrete stochastic approximation," IEEE Trans. Inform. Theory, vol. 50, no. 9, pp. 1927-1949, sep 2004. 
[141] Q. Guan, F. R. Yu, S. Jiang, and V. C. M. Leung, "Capacity-optimized topology control for MANETs with cooperative communications," IEEE Trans. Wireless Commun., vol. 10, no. 7, pp. 2162-2170, July 2011.

[142] G. R. Shorack and J. A. Wellner, Empirical Processes With Applications to Statistics. John Wiley \& Sons Inc, Apr. 1986.

[143] M. J. Neely, "Stochastic network optimization with application to communication and queueing systems," Synthesis Lectures on Communication Networks, vol. 3, no. 1, no. 1, pp. 1-211, 2010.

[144] D. S. Baum, J. Salo, M. Milojevic, P. Kyoti, and J. Hansen, "MATLAB implementation of the 3GPP Spatial Channel Model (3GPP TR 25.996)." On-line, Jan. 2005. http://www.tkk.fi/Units/Radio/scm/.

[145] R. Beard, T. McLain, D. Nelson, D. Kingston, and D. Johanson, "Decentralized cooperative aerial surveillance using fixed-wing miniature UAVs," Proc. IEEE, vol. 94, no. 7, pp. $1306-1324$, July 2006.

[146] Z. Han, A. L. Swindlehurst, and K. J. R. Liu, "Optimization of MANET connectivity via smart deployment/movement of unmanned air vehicles," IEEE Trans. Veh. Tech., vol. 58, no. 7, pp. 3533 -3546, Sept. 2009.

[147] A. Goldsmith, Wireless communications. Cambridge Press, 1996.

[148] J. I. Choi, M. Jain, K. Srinivasan, P. Levis, and S. Katti, "Achieving single channel, full duplex wireless communication," in Proc. ACM MobiCom'10, (New York, NY, USA), 2010.

[149] B. Radunovic, D. Gunawardena, P. Key, A. Proutiere, N. Singh, V. Balan, and G. Dejean, "Rethinking indoor wireless mesh design: Low power, low frequency, Full-Duplex," in Proc. of WiMesh'10, pp. 25-30, Jun. 2010.

[150] M. Duarte and A. Sabharwal, "Full-duplex wireless communications using offthe-shelf radios: feasibility and first results," in Proc. ASILOMAR'10, pp. 15581562, Nov. 2010.

[151] H. Chen, F. Yu, H. C. B. Chan, and V. C. M. Leung, "A novel multiple access scheme over multi-packet reception channels for wireless multimedia networks," IEEE Trans. Wireless Commun., vol. 6, no. 4, pp. 1501 -1511, Apr. 2007. 
[152] H. Meyr, M. Moeneclaey, and S. Fechtel, Digital communication receivers: synchronization, channel estimation, and signal processing. John Wiley \& Sons, Inc. New York, NY, USA, 1997.

[153] L. Tong, B. Sadler, and M. Dong, "Pilot-assisted wireless transmissions: general model, design criteria, and signal processing," IEEE Signal Proc. Mag., vol. 21, no. 6, no. 6, pp. 12-25, 2004.

[154] M. Ergen, D. Lee, R. Sengupta, and P. Varaiya, "Wireless token ring protocolperformance comparison with IEEE 802.11," in In Proc. IEEE ISCC'03, pp. 710-715, June 2003.

[155] J. Zhang, Z. Dziong, F. Gagnon, and M. Kadoch, "Receiver initiated mac design for ad hoc networks based on multiuser detection," in Proc. of ICST QShine'08, (ICST, Brussels, Belgium, Belgium), pp. 19:1-19:7, ICST, 2008.

[156] P. C. Weeraddana, M. Codreanu, M. Latva-aho, and A. Ephremides, "On the effect of Self-Interference cancelation in MultiHop wireless networks," EURASIP J. on Wireless Commun. \& Netw., vol. 2010, pp. 1-10, 2010.

[157] S. Adireddy and L. Tong, "Exploiting decentralized channel state information for random access," IEEE Trans. Inform. Theory, vol. 51, no. 2, no. 2, pp. 537$561,2005$.

[158] D. Tse and S. Hanly, "Linear multiuser receivers: effective interference, effective bandwidth and user capacity," IEEE Trans. Inform. Theory, vol. 45, no. 2, no. 2, pp. 641-657, 1999.

[159] IEEE Std. 802.5, "Token ring access method and physical layer specification," 1998.

[160] S. P. Bradley, Applied Mathematical Programming. Addison-Wesley, Feb. 1977.

[161] S. P. Boyd and L. Vandenberghe, Convex Optimization. Cambridge University Press, 2004.

[162] "IBM ILOG CPLEX Optimizer." website: http://www01.ibm.com/software/integration/optimization/cplex-optimizer/.

[163] J. Li, A. Sava, and X. Xie, "Simulation-based discrete optimization of stochastic discrete event systems subject to non Closed-Form constraints," IEEE Trans. Auto. Control, vol. 54, no. 12, pp. 2900-2904, Dec. 2009. 
[164] T. Camp, J. Boleng, and V. Davies, "A survey of mobility models for Ad Hoc metwork research," Wiley Wireless Commun. ES Mobile Computing, vol. 2, no. 5, no. 5, pp. 483-502, 2002.

[165] C. M. Bishop et al., Pattern Recognition and Machine Learning, vol. 1. Springer New York, 2006. 